\title{
Generalized Monodromy Conjecture in dimension two
}

\author{
ANDRÁS NÉMETHI \\ WILLEM VEYS
}

The aim of the article is an extension of the Monodromy Conjecture of Denef and Loeser in dimension two, incorporating zeta functions with differential forms and targeting all monodromy eigenvalues, and also considering singular ambient spaces. That is, we treat in a conceptual unity the poles of the (generalized) topological zeta function and the monodromy eigenvalues associated with an analytic germ $f:(X, 0) \rightarrow(\mathbb{C}, 0)$ defined on a normal surface singularity $(X, 0)$. The article targets the "right" extension in the case when the link of $(X, 0)$ is a homology sphere. As a first step, we prove a splice decomposition formula for the topological zeta function $Z(f, \omega ; s)$ for any $f$ and analytic differential form $\omega$, which will play the key technical localization tool in the later definitions and proofs.

Then, we define a set of "allowed" differential forms via a local restriction along each splice component. For plane curves we show the following three guiding properties: (1) if $s_{0}$ is any pole of $Z(f, \omega ; s)$ with $\omega$ allowed, then $\exp \left(2 \pi i s_{0}\right)$ is a monodromy eigenvalue of $f$, (2) the "standard" form is allowed, (3) every monodromy eigenvalue of $f$ is obtained as in (1) for some allowed $\omega$ and some $s_{0}$.

For general $(X, 0)$ we prove (1) unconditionally, and (2)-(3) under an additional (necessary) assumption, which generalizes the semigroup condition of NeumannWahl. Several examples illustrate the definitions and support the basic assumptions.

14B05, 14H20, 32S40; 32S05, 14H50, 14J17, 32S25

\section{Introduction}

\subsection{The Monodromy Conjecture}

The Monodromy Conjecture of Igusa, Denef and Loeser $[7 ; 8]$ is one of the most fertile conjectures in singularity theory. It relates poles of Igusa/motivic/topological zeta functions to monodromy eigenvalues. For instance, for a local analytic isolated singularity $f:\left(\mathbb{C}^{n}, 0\right) \rightarrow(\mathbb{C}, 0)$ it predicts that if $s_{0}$ is a pole of the local topological zeta function of $f$, then $\exp \left(2 \pi i s_{0}\right)$ is an eigenvalue of the local monodromy operator acting on $H^{*}\left(F_{0}, \mathbb{C}\right)$, where $F_{0}$ is the Milnor fiber of $f$. In the definition of the topological zeta function not only some invariants of the local germ $f$ are codified, but 
in a subtle way also some numerical data of the standard differential form of $\left(\mathbb{C}^{n}, 0\right)$ lifted to an embedded resolution of $f$.

The conjecture was proved for $n=2$ by Loeser (originally in the context of $p-$ adic Igusa zeta functions) in [14]. There are by now various other partial results, eg Artal Bartolo et al [3; 4], Budur, Mustaţă and Teitler [6], Lemahieu and Van Proeyen [12], Lemahieu and Veys [13], Loeser [15] and Veys [26; 30], nevertheless the conjecture resists all attacks (even for $n=3$ ). The main obstacle is the lack of a conceptual bridge connecting the two invariants, the topological zeta function and the monodromy operator; the existing proofs of the particular cases basically compute both sides independently (using their special properties) and compare the two final data.

A possible way to find a more conceptual understanding and more tools is to extend the conjecture to a larger class. This leads us to the replacement of $\left(\mathbb{C}^{n}, 0\right)$ with a singular space, and of the standard differential form with some generalization of it. Although both types of generalizations are obstructed (see Sections 1.2-1.3), the main target of the present article is to find the right such extension when the ambient space is 2-dimensional. Since the two types of generalizations are independent, and have rather different effects, in order to understand their nature, at the first discussion we separate them.

\subsection{Extending the differential form}

There is a more direct motivation for the generalization of the standard form. It is easy to see on explicit examples that for any fixed germ $f:\left(\mathbb{C}^{n}, 0\right) \rightarrow(\mathbb{C}, 0)$, and by considering the "classical" topological zeta function, not all the eigenvalues of the monodromy operator are realized; actually quite few eigenvalues are obtained this way (in general). Hence, for any fixed $f$, it is natural to try to extend in some way this set of poles, such that the same procedure would yield all eigenvalues of $f$. We expect that such a construction could reveal the conceptual bridge mentioned above. A natural way to extend poles is using the local topological zeta functions associated with the original germ $f$ and with a set of analytic differential $n$-forms $\omega$ living in $\left(\mathbb{C}^{n}, 0\right)$.

We now describe these zeta functions; they are defined in terms of an embedded resolution $\pi: \tilde{X} \rightarrow \mathbb{C}^{n}$ of $f^{-1}(0) \cup \operatorname{div}(\omega)$. We denote by $E_{i}, i \in S$, the irreducible components (exceptional divisors and strict transforms) of the inverse image $\pi^{-1}\left(f^{-1}(0) \cup \operatorname{div}(\omega)\right)$ and by $N_{i}$ and $\nu_{i}-1$ the multiplicities of $E_{i}$ in the divisor of $\pi^{*} f$ and $\pi^{*} \omega$, respectively. We put $E_{I}^{\circ}:=\left(\bigcap_{i \in I} E_{i}\right) \backslash\left(\bigcup_{j \notin I} E_{j}\right)$ for $I \subset S$. Hence, the $E_{I}^{\circ}$ constitute a stratification of $\tilde{X}$ in locally closed subsets. 
1.2.1 Definition The (local) topological zeta function of $(f, \omega)$ at $0 \in \mathbb{C}^{n}$ is

$$
Z(f, \omega ; s):=\sum_{I \subset S} \chi\left(E_{I}^{\circ} \cap \pi^{-1}(0)\right) \prod_{i \in I} \frac{1}{v_{i}+s N_{i}},
$$

where $s$ is a variable.

This definition extends naturally the definition of Denef and Loeser from [7] valid for the standard form $\omega=d x_{1} \wedge \cdots \wedge d x_{n}$. Their original proof that the corresponding expression does not depend on the chosen resolution is by describing it as a kind of limit of $p$-adic Igusa zeta functions. Later they obtained the statement as a specialization of the intrinsically defined motivic zeta functions [8]. Another technique is applying the Weak Factorization Theorem (see Abramovich et al [1] and Włodarczyk [32]) to compare two different resolutions. For arbitrary $\omega$ one can proceed analogously. Hence $Z(f, \omega ; s)$ is a well-defined invariant of the pair $(f, \omega)$.

In the literature similar generalizations are already present (see for example Artal Bartolo et al [3; 4] and Veys [29]), however they are subject to the restriction $\operatorname{supp}(\operatorname{div}(\omega)) \subset f^{-1}(0)$. In the present article we release this condition. (In the original context of $p$-adic Igusa zeta functions; see eg Loeser [14, III 3.5].)

Although $Z(f, \omega ; s)$ is a sum of "local" contributions, in this sum many local candidate poles cancel, and usually it is hard to characterize those which survive.

1.2.2 We recall that the "classical" monodromy conjecture predicts the implication

$s_{0}$ is a pole of $Z(f, \omega ; s) \Rightarrow \exp \left(2 \pi i s_{0}\right)$ is a monodromy eigenvalue of $f$,

where $\omega$ is the standard form $d x_{1} \wedge \cdots \wedge d x_{n}$. The point is that for arbitrary analytic differential forms $\omega$ this implication is in general false. Even more, in [31] the second author showed that every given monodromy eigenvalue of $f$ is induced by a pole $s_{0}$ of some $Z(f, \omega ; s)$, but in general that zeta function has other "bad" poles, not inducing eigenvalues. This shows that one can indeed generate a lot of poles, but their relationship with the eigenvalues is uncontrolled. The next program targets exactly this uncertainty via the selection of forms with compatibility properties with the monodromy operator.

Partly initiated in [31] (see also our article [19]), we propose the following program.

1.2.3 Goals Define/identify a collection of allowed analytic forms $\omega$ (depending on $f$ ) such that

(1) if $s_{0}$ is any pole of $Z(f, \omega ; s)$, where $\omega$ is allowed, then $\exp \left(2 \pi i s_{0}\right)$ is a monodromy eigenvalue of $f$, 
(2) the standard form $\omega=d x_{1} \wedge \cdots \wedge d x_{n}$ is allowed,

(3) every monodromy eigenvalue of $f$ is obtained as in (1) for some allowed $\omega$ and some $s_{0}$.

A few remarks are in order. First, note that (1) and (2) imply the classical Monodromy Conjecture. Furthermore, (1) and (3) combined show that the set of eigenvalues of $f$ coincides with the set $\exp (2 \pi i P)$, where $P$ runs over all the poles of the zeta functions of $f$ and all allowed forms.

Note also that the "size" of the wished allowed forms is obstructed by both conditions (1) and (3). A larger set is obstructed more by (1), while if this set is too small then it may not realize in (3) all eigenvalues. In particular, its construction really requires a conceptual understanding of the geometry of the pole-eigenvalue bridge mentioned above.

Let us briefly support our goal by comparing with a more classical context. Recall that the topological zeta function is a kind of avatar of the $p$-adic Igusa zeta function, which is the meromorphic continuation of a $p$-adic integral associated to a $p$-adic function germ $f$ (with complex parameter/variable $s$ ). We could rephrase the above also for that zeta function, again with complex parameter/variable $s$. For the analogous complex integral associated to a complex function germ $f$, and involving compactly supported $C^{\infty}$ forms $\omega$, there are general theorems by Malgrange [16], Kashiwara [10] and Barlet [5], claiming the analogous statements of the goals above. Roughly, if $s_{0}$ is any pole of the zeta function of $f$ and any compactly supported $C^{\infty}$ form $\omega$, then $\exp \left(2 \pi i s_{0}\right)$ is a monodromy eigenvalue of $f$, and all eigenvalues are obtained this way. Our "standard form" can be compared with a $C^{\infty}$ form that is nonvanishing. For a detailed explanation and comparison, we refer to the introduction of [31]. However, the "exact comparison" in general fails, and it is still hidden what the analogue of compactly supported $C^{\infty}$ forms is in the holomorphic category.

One of the main results of this paper is an identification of a set of allowed forms realizing the Goals (1)-(3) above for $n=2$, that is for an arbitrary plane curve germ $f$. Our technique is to consider the so-called splice diagram of $f$, and its splice decomposition in star-shaped pieces. It is not difficult (and reasonably conceptual) to define the allowed forms and realize our goal when the diagram of $f$ itself is star-shaped; we then use this as inspiration for the general case, identifying allowedness "locally", that is, on all star-shaped subdiagrams; cf Section 4.

\subsection{Extension to a singular ambient space}

An important new feature in this paper is generalizing Goals 1.2.3 to a singular setting. More precisely, we will consider an analytic function germ $f$ defined on a normal 
surface germ $(X, 0)$ and study the analogue of the Goals (1)-(3) for this $f$. First we must identify the relevant (generalized) topological zeta function for such a pair $(X, f)$ and for an analytic form $\omega$. In fact, in all our combinatorial arguments, we will replace $(f, \omega)$ by two Weil divisors (for details and motivation see Section 1.5.1).

Of course, in order to have a well-defined analogue of Goals 1.2.3(2), we need to consider Gorenstein germs, which guarantees the existence of a "standard form". If the ambient space is two-dimensional, the Gorenstein condition simplifies at topologi$\mathrm{cal} /$ combinatorial level to the numerically Gorenstein condition, which is automatically guaranteed, for example, if the link is an integral homology sphere.

Nevertheless, we will need some further combinatorial restrictions. There is an example of Rodrigues [24] indicating that the "naive" extension of the Monodromy Conjecture to the Gorenstein singular setting might be obstructed. This example produces a set of integers $\left\{v_{i}\right\}_{i}$ and $\left\{N_{i}\right\}_{i}$ associated with the exceptional divisors (or, with vertices of the plumbing graph) which topologically are not obstructed to be the multiplicities of the standard form and of an analytic germ $f$, and they produce a counterexample to the Monodromy Conjecture. In Example 7.4.25 we even construct another such example involving a Gorenstein surface singularity with unimodular dual graph. One of the following two possibilities can solve this situation in order to have a chance for a positive continuation: either we impose some additional topological restrictions which eliminate any such counterexample, or we try to show that the analytic realization of the analytic germ (in the presence of the Gorenstein structure) guarantees these additional needed topological restrictions. The second possibility looks very difficult and is hopeless with the present tools of the theory, and it is not the goal of the present article to attack it. Therefore, in order to have an extended version, we stay with the first possibility.

The additional restriction we impose, in fact, is very natural; it is a modification of the semigroup condition of Neumann and Wahl [20], adapted to the present situation and to "divisors supported on a graph". This condition is automatically satisfied if the ambient space is smooth (and in several other cases too). This also emphasizes a subtle connection between the semigroup condition and the Monodromy Conjecture.

Our second main result extends the combinatorial definition of the allowed forms to the singular surface case when the link of the ambient space is an integral homology sphere, and establishes for them Goal (1) unconditionally, and (2) and (3) under the semigroup condition.

Allowed forms are again defined via the same local picture of the splice components. We emphasize that the definition of the allowed forms, the generalized semigroup condition, and the whole proof of Goals 1.2.3 is combinatorial: one uses only the Weil 
divisors of the functions and forms (and their analytic realizations will not be involved). In Section 1.5.1 we give some details about the formalism of zeta functions associated with Weil divisors, and in Section 1.5.3 we motivate the definition of allowed forms. An ambient germ with integral homology sphere link will be abbreviated by IHS germ.

\subsection{Plan of the paper and new results}

In Section 2 we recall the classical notion of splice diagrams for surface germs and for functions/divisors on them, and we incorporate in the picture also differential forms (and their generalizing divisors). Here we also introduce the extension of the semigroup condition of Neumann and Wahl in the presence of a divisor. Section 3 treats the concept of splicing of these diagrams. The relevant "splice formulae" are well known for functions on surface germs; we develop them for differential forms. Then we use these formulae to derive a splice formula for the topological zeta function; see Theorem 3.2.4. In Section 4 we define our allowed forms/divisors and investigate their crucial properties concerning restriction and extension along (sub)diagrams. In Section 5 we show that any pole of the zeta function associated to a function $f$ and any allowed form/divisor induces a monodromy eigenvalue of $f$ (first goal). The second goal (standard form is allowed) and third goal (any eigenvalue is induced by a pole of a zeta function of an allowed form) are proved for plane curve germs (unconditionally) in Section 6. Finally their generalized versions for functions on IHS germs (under the semigroup condition) are proven in Section 7.

Additionally, we list several examples in order to make the article more readable, and in order to emphasize the role of several key points in definitions or about needed restrictions. For example, Examples 4.1.7 and 7.4.24 show that the semigroup condition is necessary to have Goals (2) and (3), respectively, (at least in any topological treatment), while the discussion from Example 7.4.25 shows that if we drop the IHS assumption about the link of the ambient space we need to treat a much stronger (and presumably more technical) notion replacing the semigroup condition.

\subsection{Some more details and motivations}

Here we present the key motivations for the major restrictions and constructions of the article as a separate guide.

\subsubsection{Topological zeta function on a singular ambient surface We first explain} what the topological zeta function is on a singular ambient surface associated with two Weil divisors. 
There have been various generalizations of topological and motivic zeta functions to singular ambient varieties $X$ instead of $\mathbb{C}^{n}$ (see for example Veys [29]) and specifically for surfaces (see Rodrigues and the second author's works [24; 25; 28]). Before introducing the ones we will use, note that the zeta function $Z(f, \omega ; s)$ of Definition 1.2.1 depends in fact only on the effective divisors $F:=\operatorname{div}(f)$ and $W:=\operatorname{div}(\omega)$ on $\left(\mathbb{C}^{n}, 0\right)$, and not on the actual function $f$ and form $\omega$. In terms of these divisors, the numerical data $N_{i}$ and $v_{i}-1$ above are given as the multiplicities of $E_{i}$ in the divisors $\pi^{*} F$ and $K_{\pi}+\pi^{*} W$, respectively, where $K_{\pi}$ is the relative canonical divisor of $\pi$.

Therefore, it is natural to associate in a singular setting a topological zeta function to two Weil divisors on $X$, in terms of an embedded resolution $\pi$ of the union of their supports. For this we should be in a situation where there is a natural notion of pullback of Weil divisors, and where the relative canonical divisor $K_{\pi}$ exists. Both conditions are satisfied when $(X, 0)$ is an arbitrary normal surface germ (for the pullback see Section 2.2.2, while for $K_{\pi}$ see Equation (2.3.2)). Let $F:=\sum_{j \in J} N_{j} E_{j}$ be an effective nonzero Weil divisor, and $W:=\sum_{j \in J}\left(v_{j}-1\right) E_{j}$ an arbitrary Weil divisor on $X$, where $E_{j}, j \in J$, are (finitely many) irreducible Weil divisors. We only require that $\left(N_{j}, v_{j}\right) \neq(0,0)$ for $j \in J$, that is, a component $E_{j}$ that appears in $W$ with multiplicity -1 must appear in the support of $F$.

Let $\pi: \tilde{X} \rightarrow X$ be an embedded resolution of $\operatorname{supp}(F) \cup \operatorname{supp}(W)$. We denote again by $E_{i}, i \in S$, the irreducible components of its inverse image, and by $N_{i}$ and $v_{i}-1$ the multiplicities of $E_{i}$ in the divisors $\pi^{*} F$ and $K_{\pi}+\pi^{*} W$, respectively. Note that the $N_{i}$ and $v_{i}$ of exceptional components $E_{i}$ are in general rational numbers. The (local) topological zeta function of $(F, W)$ at $0 \in X$, denoted as $Z(F, W ; s)$, is defined by the same formula as in Definition 1.2.1. It is straightforward to verify that this expression does not depend on the chosen resolution $\pi$.

In our study below we will assume that the link of $X$ is an integral homology sphere; then in particular all $N_{i}$ and $v_{i}$ are integers. In such a context, more useful for us will be a formula for $Z(F, W ; s)$ in terms of the splice diagram associated to $(F, W)$; see Section 2.4. Roughly, the splice diagram is obtained from the dual minimal embedded resolution graph of $\operatorname{supp}(F) \cup \operatorname{supp}(W)$ by collapsing the strings to edges and modifying the decorations by a system of data which describes more trustworthily the needed linking numbers.

This description allows us to determine a splice formula showing the "almost additivity" of $Z(F, W ; s)$ with respect to the splice decomposition of the diagrams. This is another novelty of the article, which becomes a crucial tool in the main proofs.

1.5.2 Restrictions regarding $W$ It is an easy fact that in the resolution graph exceptional components of valency 1 or 2 do not contribute to the actual poles of the zeta 
function, and that those of valency at least 3 (corresponding to the nodes in the splice diagram) in general do contribute to the poles. Since precisely those last components contribute to the monodromy eigenvalues, it is very reasonable to restrict from the start the support of the desired allowed $W$ as follows. The map $\pi$ should be also an embedded resolution of $f^{-1}(0) \cup \operatorname{supp}(W)$, and more precisely $\operatorname{supp}(W)$ should consist only of components coinciding with components of $f$ and components whose strict transform intersects the exceptional locus in a component of valency 1 ; moreover such a component of valency 1 must intersect at most one component of $W$. In this way, one does not create new exceptional components of valency at least 3 and does not transform those of valency 1 or 2 into components of valency 3 , what would probably create undesired new poles. This restriction has a similar formulation in terms of splice diagrams too.

1.5.3 Allowed forms/divisors Next, we give the idea of the definition of allowed forms/divisors associated with a fixed function $f$ (or Weil divisor $F$ ), with some motivation. It will be illustrated via the plane curve germ given by the function $f=\left(y^{d_{1}}-x^{d_{2}}\right)\left(y^{d_{1}}+x^{d_{2}}\right)$, where $d_{1}>1, d_{2}>1$ and $\operatorname{gcd}\left(d_{1}, d_{2}\right)=1$. Below is its star-shaped splice diagram with node $E$, where the dashed arrows indicate the support of the strict transform of $W=\operatorname{div}(\omega)$, and the decorations along these arrows are the multiplicities of its components (for details, see Section 2).

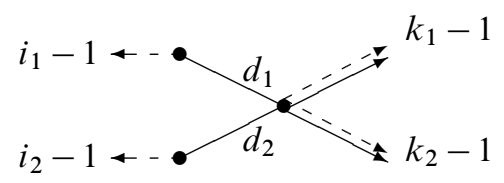

Then $N=2 d_{1} d_{2}$ is the vanishing order of $f$ along the node $E$.

(i) By A'Campo's formula, the monodromy eigenvalues of $f$ are, besides the trivial eigenvalue 1 , precisely the roots of the polynomial

$$
\Lambda(t)=\frac{\left(t^{N}-1\right)^{2}}{\left(t^{N / d_{1}}-1\right)\left(t^{N / d_{2}}-1\right)} .
$$

These are all $N$-th roots of unity that are not simultaneously $\left(N / d_{1}\right)$-th and $\left(N / d_{2}\right)-$ th roots of unity; in other words all $\exp \left(2 \pi i \frac{u}{N}\right)$ for which $d_{1} \nmid u$ or $d_{2} \nmid u$.

(ii) Using Section 2.4 one has

$$
Z(f, \omega ; s)=\frac{1}{v+s N}\left(-2+\frac{d_{1}}{i_{1}}+\frac{d_{2}}{i_{2}}+\frac{1}{k_{1}+s}+\frac{1}{k_{2}+s}\right),
$$


where (see (2.3.5))

$$
v=d_{1} d_{2}\left(k_{1}+k_{2}-2\right)+d_{2} i_{1}+d_{1} i_{2} .
$$

We now investigate the candidate pole $s_{0}:=-v / N$ of the zeta function. If $s_{0}$ is a pole of order one, one easily verifies that its residue $\mathcal{R}$ is not identically zero as function in the four variables $i_{1}, i_{2}, k_{1}, k_{2}$. Hence $s_{0}$ is a pole of $Z(s)$ as soon as the algebraic equation $\mathcal{R}=0$ is not satisfied. It is also straightforward to compute that $\mathcal{R}$ is identically zero in $k_{1}$ and $k_{2}$ if $i_{1}=d_{1}$ and $i_{2}=d_{2}$, and that generally $\mathcal{R}$ is not identically zero otherwise.

(iii) With respect to Goal (1), if we wish to put only "necessary" restrictions to realize it, the following is a very natural choice for allowed $W$. Note first that $d_{\ell} \mid v$ if and only if $d_{\ell} \mid i_{\ell}$ (by (1.5.4)). We call $W$ allowed if the following condition on $i_{1}$ and $i_{2}$ is satisfied: if $d_{1} \mid i_{1}$ and $d_{2} \mid i_{2}$, then $i_{1}=d_{1}$ and $i_{2}=d_{2}$. (There are no conditions on $k_{1}$ and $k_{2}$.) Therefore, for $W$ allowed, if $\exp \left(2 \pi i s_{0}\right)$ is not a root of $\Lambda$, then $d_{1} \mid i_{1}$ and $d_{1} \mid i_{2}$, hence $\mathcal{R}=0$.

Moreover with this definition the divisor $W=0$, corresponding to $i_{1}=i_{2}=k_{1}=$ $k_{2}=1$, is clearly allowed (Goal (2)), and Goal (3) is also satisfied. Indeed, fix a root $\exp \left(2 \pi i \frac{u}{N}\right)$ of $\Lambda(t)$. Since the numbers $d_{1}$ and $d_{2}$ are coprime, there exist integers $k_{1}, k_{2}, i_{1}, i_{2}$ (all positive if we desire so) such that $v$ in (1.5.4) satisfies $v \equiv u \bmod N$. The restrictions on the given $u$ imply that $d_{1} \nmid i_{1}$ or $d_{2} \nmid i_{2}$. Hence the constructed $W$ is allowed.

One can carry out without too much effort a similar analysis for any plane curve germ $f$, or, more generally, for any function $f$ on an IHS germ for which the splice diagram is star-shaped, identifying allowed forms $\omega$ /divisors $W$ satisfying our Goals 1.2.3; see Definition 4.1.1. For arbitrary $f$ the situation is at first sight combinatorially hopeless. Nevertheless, for them we use the concept of splicing of a general splice diagram into star-shaped building blocks, and we ask that the "restriction" of the desired $W$ to any such star-shaped building block satisfies the "natural" (already identified) conditions of allowedness.

Though conceptually appealing, it is not clear from the start that such a "local" definition of allowedness will do the job, a priori it is even not obvious that allowed forms/divisors exist on arbitrary diagrams. It will turn out that there are plenty of them, and at the end the proof of the first goal will be (combinatorially) quite conceptual.

We still want to mention one important point regarding the introduction of Weil divisors discussed in Section 1.5.1. In the singular setting, in order to deal with our goals concerning the zeta functions associated to a given function $f$, by our inductive splicing strategy, we consider the restriction of the divisor of $f$ to the star-shaped 
building blocks; the point is that usually the analytic realization of them is a difficult issue deviating from the original main objective. This shows that the introduction of Weil divisors is even necessary.

Acknowledgements The first author is partially supported by OTKA Grants. The second author is partially supported by FWO-Flanders project G.0318.06.

\section{Splice diagrams associated to normal surface singularities}

\subsection{Splice diagrams of surface-germs}

Let $(X, 0)$ be the germ of a complex normal surface singularity, and $M$ be its link. It is well-known that $M$ is an oriented plumbed 3-manifold, and any dual resolution graph might serve as a plumbing graph for $M$. Let $\pi: \tilde{X} \rightarrow X$ be a good resolution, that is, the exceptional divisor $E:=\pi^{-1}(0)$ is a normal crossings divisor on the smooth complex surface $\tilde{X}$. The topology of $\pi$ is codified in the dual graph $G=G_{\pi}(X)$ associated with the irreducible components $\left\{E_{i}\right\}_{i}$ of $E$ : each $E_{i}$ determines a vertex of $G$ with genus decoration $\left[g\left(E_{i}\right)\right]$ and self-intersection $\left(E_{i}, E_{i}\right)$, while the edges of $G$ correspond to intersection points $E_{i} \cap E_{j}$; cf Laufer [11]. Since $X$ is normal, $G$ is connected. Let $I(G)$ be the negative definite intersection form $\left(E_{i}, E_{j}\right)_{i, j}$. By plumbing construction one recovers from $G$ both $M$ and (the $C^{\infty}$-type of) $(\tilde{X}, E)$.

We recall that $M$ is a rational homology sphere if and only if $G$ is a tree and $g\left(E_{i}\right)=0$ for all $i$. Moreover, $M$ is an integral homology sphere if additionally $\operatorname{det}(-I(G))=1$.

If $M$ is an integral homology sphere, then $G$ can equivalently be codified in a more condensed form via its splice diagram $\Gamma=\Gamma_{\pi}(X)$; cf Eisenbud and Neumann [9]. The diagram $\Gamma$ is the tree obtained from $G$ by replacing each maximal string of $G$ by a single edge. Hence, $\Gamma$ is homeomorphic to $G$, but it has no vertices of valency 2 . Its vertices are either nodes (of valency $\geq 3$ ) or ends/boundary vertices (of valency 1 ); they correspond to the nodes/rupture vertices and boundary vertices of $G$ with valencies $\geq 3$ and 1, respectively. The decorations of $\Gamma$ are as follows. At each node $v$ of $\Gamma$ one inserts a weight $d_{v e}$ on each incident edge $e$. Let $G_{v e}$ be the connected component of $G \backslash\{v\}$ "in the direction of $e$ ", then $d_{v e}:=\operatorname{det}\left(-I\left(G_{v e}\right)\right)$. It is proved in [9, Chapter V] that the decorated graphs $G$ and $\Gamma$ determine each other.

The decorations $\left\{d_{v e}\right\}_{v, e}$ of $\Gamma$ satisfy the next compatibility conditions:

$$
\left\{\begin{array}{l}
\text { (a) } d_{v e} \geq 1 . \\
\text { (b) }\left\{d_{v e}\right\}_{e} \text { are pairwise coprime integers for any fixed node } v . \\
\text { (c) any "edge determinant" } q_{e} \text { is positive. }
\end{array}\right.
$$


Part (c) means the following: for any fixed edge $e$ with end-nodes $v$ and $w$, let the decorations at $v$ be $d_{v e}$ and $\left\{d_{v e_{i}}\right\}_{i}$, and similarly $d_{w e}$ and $\left\{d_{w e_{j}^{\prime}}\right\}_{j}$ at $w$. Then (see [9, Section 24])

$$
q_{e}:=d_{v e} d_{w e}-\prod_{i} d_{v e_{i}} \prod_{j} d_{w e_{j}^{\prime}}>0 .
$$

If $\pi$ is the minimal good resolution, then $\Gamma$ also is minimal, in the sense that all the decorations $d_{v e}$ are strictly greater than 1 , provided that $e$ connects $v$ with a boundary vertex. If $G$, or $\Gamma$, is not minimal, then such a restriction does not hold. By "splice calculus", one can delete such an edge with decoration 1 and the supported boundary vertex, getting a new equivalent diagram. All these equivalent diagrams represent the same 3-manifold $M$. The nodes (and the corresponding star-shaped subgraphs around them) correspond exactly to the (minimal or nonminimal) Jaco-Shalen-Johannson decomposition of $M$ (depending on the minimality of $\Gamma$ ), each star-shaped subgraph describing a Seifert piece.

2.1.3 The diagram $\Gamma$ (or $G$ ) contains the same amount of information as the link $M$, hence working with it we disregard completely the analytic structure of $(X, 0)$. In the sequel we regard $\Gamma$ as an abstract splice diagram which satisfies (2.1.1). In fact, any such diagram can be realized by some singularity link, but the corresponding analytic structure(s) can be hard to determine and are irrelevant from the point of view of many invariants.

In this correspondence, in fact, there is an "easy case", namely when $(X, 0)$ is smooth and $M$ is the 3-sphere $S^{3}$ : this is happening if and only if in any (maybe nonminimal) splice diagram which represents them the following fact holds: for any node $v$ at most two of the integers $\left\{d_{v e}\right\}_{e}$ can be strictly greater than 1 (that is, any Seifert piece is an $S^{3}$ ).

2.1.4 Semigroup condition for $\Gamma$ It is convenient to introduce some other combinatorial invariants of a splice diagram $\Gamma$ as well. If $v$ and $w$ are two vertices of $\Gamma$, we set $\ell_{v w}$ for the product of the edge weights that are adjacent to, but not on, the path from $v$ to $w$. Furthermore, for each node $v$, let $d_{v}$ be the product of edge weights adjacent to $v$.

For any node $v$ and adjacent edge $e$, let $\Gamma_{v e}$ be the connected component of $\Gamma \backslash\{v\}$ in the direction of $e$. We say, following [20], that the node $v$ and adjacent edge $e$ satisfy the semigroup condition if

$d_{v}$ is in the semigroup generated by the $\ell_{v w}$, where $w$ is a boundary vertex of $\Gamma$ in $\Gamma_{v e}$. 
By definition, a splice diagram $\Gamma$ satisfies the semigroup condition, if all pairs $(v, e)$ as above satisfy the semigroup condition.

\subsection{Splice diagrams of function-germs/divisors}

Assume that $f:(X, 0) \rightarrow(\mathbb{C}, 0)$ is the germ of an analytic function on an IHS germ $(X, 0)$. If $\pi: \tilde{X} \rightarrow X$ is an embedded resolution of the pair $\left(X, f^{-1}(0)\right)$, then the topology of $f$ is described by the embedded resolution graph of $f$. This consists of the dual graph $G_{\pi}(X, f)$ of the exceptional divisors decorated by the self-intersections, and supplemented by the following data: each irreducible component of the strict transform intersecting an irreducible exceptional divisor $E_{i}$ is codified by an arrowhead supported by that vertex of $G$ which corresponds to $E_{i}$. Additionally, each vertex and arrowhead inherits a multiplicity decoration, the vanishing order of $f \circ \pi$ along the corresponding irreducible divisor.

Clearly, the $\operatorname{divisor} \operatorname{div}(f \circ \pi)$ on $\tilde{X}$ is a principal divisor, hence $\left(\operatorname{div}(f \circ \pi), E_{i}\right)=0$ for any $i$. This (and the fact that $\operatorname{det}(I(G)) \neq 0$ ) shows that all the multiplicities of the strict transforms of $f=0$ determine $\operatorname{div}(f \circ \pi)$ completely (compare with (2.2.3)). In fact, this property identifies $\operatorname{div}(f \circ \pi)$ as the pullback of the divisor $f=0$ on $X$.

More generally, a divisor $F$ on $\tilde{X}$ supported on $E$ and on some noncompact transversal slices of $E$ is called $P$-divisor of $\tilde{X}$ if $\left(F, E_{i}\right)=0$ for any $i$. If we start with an arbitrary Weil divisor $F^{\prime}$ on $X$ and $\pi$ is an embedded resolution of the pair $\left(X, F^{\prime}\right)$, then there is an unique $P$-divisor $F$ on $\tilde{X}$ whose arrow-multiplicities agree with the multiplicities of the components of $F^{\prime}$. This $F$ will be called the pullback of $F^{\prime}$. On the other hand, if $F$ is a $P$-divisor on $\tilde{X}$, projecting down its noncompact components (by keeping their multiplicities) we get a Weil divisor $F^{\prime}$ on $X$ such that $F$ is the pullback of $F^{\prime}$. Hence, $F$ and $F^{\prime}$ determine each other (thus we will sometimes write $F$ for both of them).

A $P$-divisor is codified in the graph $G$ similarly as the principal divisors via its arrowheads and multiplicity system, and it is uniquely determined by the arrowheads and their multiplicities. This pair is denoted by $G_{\pi}(X, F)$.

In all of our topological-combinatorial discussions, we regard $G_{\pi}(X, F)$ as a combinatorial object, a plumbing representation of a pair $\left(M, M \cap F^{\prime}\right)$. We do not ask the analytic realization of $F^{\prime}$ as a principal divisor (and even if we do in some discussions, we always consider analytic realizations and not algebraic ones).

2.2.1 The splice diagram $\Gamma=\Gamma_{\pi}(X, f)$ associated with $(X, f)$ (or more generally, $\Gamma_{\pi}(X, F)$ associated with $\left.G_{\pi}(X, F)\right)$ is constructed similarly as above, but now the 
nodes are those vertices which have valency $\geq 3$ including the edges supporting arrowheads. Moreover, the new splice diagram contains arrowheads and multiplicity decorations as well (see [9]).

For the arrowheads of the minimal splice diagram we use the following principle. If an arrowhead $a$ of $G$ is supported by a vertex $v$ of $G_{\pi}(X, F)$ with valency $\geq 3$ (including the edges supporting arrowheads) then $v$ becomes a node of $\Gamma$ and $a$ becomes an arrowhead of $\Gamma$ supported by $v$. The weight of the edge at $v$ supporting such an arrowhead is either 1 or is missing. Next, assume that the arrowhead $a$ of $G_{\pi}(X, F)$ is supported by a vertex $v$ of $G$ of valency 2 . This means that $v$ is a boundary vertex of $G_{\pi}(X)$ with an arrowhead. If we forget about the arrowheads and we determine $\Gamma$ from $G_{\pi}(X)$, then $v$ becomes a boundary vertex of $\Gamma_{\pi}(X)$. Then, reconsidering the arrowheads, this boundary vertex in $\Gamma_{\pi}(X, F)$ is replaced by an arrowhead. Summed up: in minimal diagrams, all the arrowheads of $\Gamma_{\pi}(X, F)$ are supported by nodes, nevertheless they have two different interpretations: if the weight of an edge (at the node $v$ ) supporting an arrowhead is $\geq 2$ then the corresponding strict transform intersects the corresponding boundary curve of $G$, while if the weight is 1 (or it is missing) then the strict transform intersects that exceptional component which corresponds to the node. (In the language of knots: weight $\geq 2$ gives a special Seifert fiber, while weight 1 a generic Seifert fiber in the corresponding Seifert piece.)

Nevertheless, sometimes we also allow nonminimal representations (which appear naturally when we splice the diagrams). Namely, the following calculus provides equivalent diagrams:
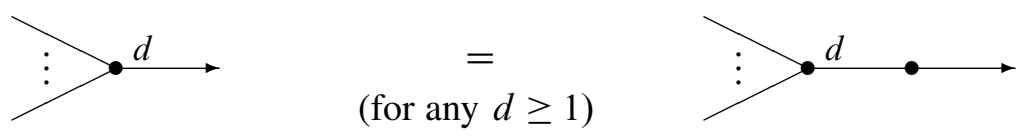

Also, $\Gamma_{\pi}(X, F)$ inherits the multiplicity of each arrowhead and node from $G_{\pi}(X, F)$ (with the same geometric interpretation). In the case of principal divisors, $f$ defines an isolated singularity if and only if all arrowhead-multiplicities are 1.

We will use the notation $\mathcal{V}$ for the vertices, $\mathcal{N}$ for the nodes, $\mathcal{B}$ for the boundary vertices and $\mathcal{E}$ for the edges of $\Gamma_{\pi}(X, F)$. Moreover, we call special edges those connecting two nodes, denoted by $\mathcal{E}^{s}$. The arrowheads will be denoted by $\mathcal{A}_{F}$, the multiplicities by $\left(N_{w}\right), w \in \mathcal{V} \cup \mathcal{A}_{F}$.

Again, we can regard $\Gamma_{\pi}(X, F)$ as an abstract graph, we do not ask about the analytic realization of the pair $(X, F)$ (although, if the graph satisfies (2.1.1) and $N_{a}>0$ for all $a \in \mathcal{A}_{F}$, then some analytic realization exists; cf [9, Section 24].) 
2.2.2 Recall that for any $P$-divisor the multiplicities of the arrowheads (and the combinatorics of the splice diagram without the other multiplicities) determine all the multiplicities of the vertices. (In the case of the graph $G$, this is done via $I(G)^{-1}$.) In the language of $\Gamma$ one has the following. Let $v$ be a fixed vertex, and let $a$ be an arrowhead. Then define $\ell_{v a}$ as the product of the edge weights that are adjacent to, but not on, the path from $v$ to $a$. Then the multiplicity $N_{v}$ of any vertex $v$ is given by (cf $[9$, Section 10])

$$
N_{v}=\sum_{a \in \mathcal{A}_{F}} N_{a} \ell_{v a}
$$

In particular, if $F^{\prime}=\sum_{a} N_{a} F_{a}^{\prime}$ is a Weil divisor on $X$ with the $F_{a}^{\prime}$ irreducible, and $\left\{F_{a}\right\}_{a \in \mathcal{A}_{F}}$ are the strict transforms and $F_{v}=E_{v}$ the exceptional curves, then the pullback $F=\pi^{*}\left(F^{\prime}\right)$ of $F^{\prime}$ is represented in the splice diagram by $\sum_{v \in \mathcal{A}_{F} \cup \mathcal{V}} N_{v} F_{v}$.

2.2.4 Compatibility conditions The splice diagram $\Gamma_{\pi}(X, F)$ also satisfies the compatibility conditions (2.1.1). If one deletes all the arrowheads and decorations of $\Gamma_{\pi}(X, F)$ associated with $F$ we recover a possible (maybe nonminimal) splice diagram of $X$. Nevertheless, by this simplification, some of the nodes might disappear.

2.2.5 Semigroup condition for $\Gamma_{\pi}(X, F)$ In the presence of a divisor, the semigroup condition Section 2.1.4 will be modified as follows. We say that $\Gamma_{\pi}(X, F)$ satisfies the semigroup condition if all pairs $(v, e)$ satisfy Section 2.1.4, provided that $v$ is a node with adjacent edge $e$, and the connected part of $\Gamma_{\pi}(X, F) \backslash\{v\}$ in the direction of $e$ contains no arrowheads.

2.2.6 Remark (1) The splice diagram associated with the minimal embedded resolution of a plane curve singularity always satisfies the semigroup condition. Indeed, there is only one subdiagram (see below), where the condition is not satisfied trivially: the nodes of this subdiagram are not sitting on geodesic paths connecting two arrowheads of the diagram.

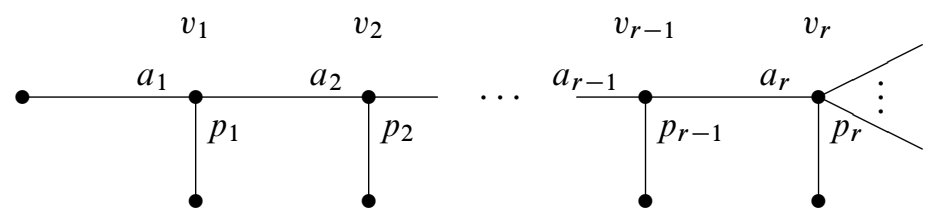

For this part, the semigroup condition follows from the positivity of the edge determinants and the fact (used iteratively several times) that for coprime positive integers $a$ and $p$, each integer larger than $a p$ belongs to the semigroup generated by $a$ and $p$. (For a more general argument, see Section 7.1.) 
(2) If $F=0$, we recover the semigroup condition of Section 2.1.4. On the other hand, the semigroup condition of $\Gamma_{\pi}(X, F)$, and of the diagram obtained from $\Gamma_{\pi}(X, F)$ by deleting the information regarding $F$, are independent. This fact is exemplified next. Note that in the semigroup condition the position of the arrowheads is important, while the multiplicity system of $F$ is irrelevant, hence we will omit the multiplicities from the next diagrams.

(3) It is possible that $\Gamma_{\pi}(X)$ does not satisfy the semigroup condition, but $\Gamma_{\pi}(X, F)$ for some $F$ does. Take for instance any $P$-divisor $F$ with enough arrowheads such that $\Gamma \backslash\{v\}$ contains an arrowhead in the direction of $e$ for each pair $(v, e)$.

(4) On the other hand, it is possible that $\Gamma_{\pi}(X)$ satisfies the semigroup condition, but $\Gamma_{\pi}(X, F)$ does not, due to the appearance of the new nodes which support the arrowheads. Take for example the following resolution graph and the corresponding splice diagram.
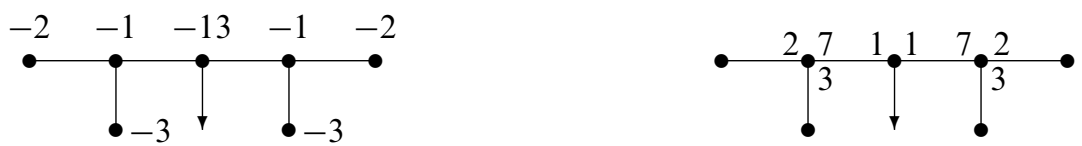

Then $\Gamma_{\pi}(X, F)$ does not satisfy the semigroup condition at the central node, although if we delete the arrowhead then $\Gamma_{\pi}(X)$ does since that node disappears.

\subsection{Splice diagrams and differential forms}

We still consider an IHS singularity $(X, 0)$, and a function germ $f$ or a nonzero effective Weil divisor $F^{\prime}$ on $X$. We fix an embedded resolution $\pi: \tilde{X} \rightarrow X$ of $\left(X, f^{-1}(0)\right)$ or $\left(X, F^{\prime}\right)$ as in Section 2.2.

Next, we also wish to incorporate in the picture a differential (meromorphic) 2-form $\omega$ or a Weil divisor $W$. The basic models for us are the following situations.

- Assume that $(X, 0)$ is smooth, hence $f$ determines a plane curve singularity. Then classically one considers $\omega_{0}=d x \wedge d y$ (for some local coordinates $(x, y)$ of $(X, 0))$, or, more generally, $\omega=g \omega_{0}$ for some local analytic germ $g$ on $(X, 0)$. In this case the pullback $\widetilde{\omega}:=\pi^{*}(\omega)$ is clearly holomorphic on $\widetilde{X}$.

- Generalizing to the singular setting, we assume that $(X, 0)$ is Gorenstein and that $\omega_{0}$ is a nowhere vanishing holomorphic 2-form on $X \backslash\{0\}$. Then, for any holomorphic germ $g$ on $X$, the pullback $\widetilde{\omega}:=\pi^{*}\left(g \omega_{0}\right)$ has a meromorphic extension over the exceptional curve. If $(X, 0)$ is rational then it is holomorphic, otherwise it might have poles. 
We will enrich the diagrams with the vanishing orders of $\operatorname{div}(\widetilde{\omega})$ along the corresponding irreducible divisors. Again, this only depends on $\operatorname{div}(\widetilde{\omega})$, not on $\widetilde{\omega}$ itself; so we rather incorporate from the start a Weil divisor $W^{\prime}$ on $X$. In the special cases above $\operatorname{div}(\omega)=\operatorname{div}(g)=W^{\prime}$ and $\operatorname{div}(\widetilde{\omega})=K_{\pi}+\pi^{*} W^{\prime}$. Also, it will be natural in our context to restrict the possible support of $W^{\prime}$; we assume that the following facts hold:

(1) $\pi$ is also an embedded resolution of $\operatorname{supp}\left(F^{\prime}\right) \cup \operatorname{supp}\left(W^{\prime}\right)$.

(2) if $W^{\prime}:=\sum_{k}\left(i_{k}-1\right) W_{k}^{\prime}$ is the irreducible decomposition of $W^{\prime}$, then the strict transform of each $W_{k}^{\prime}$ either is identical with the strict transform $F_{a}$ of one of the $F_{a}^{\prime}$, or, it intersects a boundary component of $G_{\pi}(X, F)$. Moreover, each boundary component can intersect at most one of the strict transforms of the $W_{k}^{\prime}$.

We denote the $P$-divisor $\pi^{*}\left(W^{\prime}\right)$ by $W$. In our diagrams, the strict transform $W_{k}$ of a $W_{k}^{\prime}$ that intersects $E_{i}$ will be denoted by a dashed arrowhead, attached to the vertex corresponding to $E_{i}$. If $W_{k}$ agrees with one of the $F_{a}$, then the dashed arrowhead doubles the associated ordinary arrowhead, while if a boundary vertex does not support any ordinary arrowhead but supports a $W_{k}$, then its dashed arrow is attached to this vertex. We will denote by $\mathcal{A}_{W}$ the dashed arrowheads of $W$. The "double" arrowheads are given by $\mathcal{A}_{F} \cap \mathcal{A}_{W}$.

Again, we will identify the following diagrams:

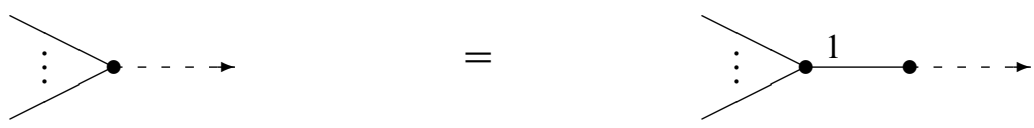

Finally, we have to add to the decorations the multiplicities of the components of $K_{\pi}+W$. By technical (and traditional) reasons, the multiplicity of a dashed arrowhead is denoted as above by $i_{a}-1$, while the multiplicity of a vertex $v$ by $v_{v}-1$. Recall that, in general, we do not impose for the integers $i_{a}$ and $v_{v}$ to be positive. We denote this enriched diagram as $\Gamma_{\pi}(X, F, W)$.

Note that in the three different levels $\Gamma_{\pi}(X), \Gamma_{\pi}(X, F)$ and $\Gamma_{\pi}(X, F, W)$, the valencies of a fixed vertex $v$ of $\Gamma$ are not the same; these three valencies will be denoted by $\delta_{v}, \delta_{v}^{\prime}$ and $\delta_{v}^{\prime \prime}$ respectively.

2.3.1 In order to identify the coefficients $v_{v}-1$ of $K_{\pi}+W$, we only must describe the coefficients of the $E_{v}$ in $K_{\pi}$, since those of $W$ are as in Section 2.2.2.

The divisor $K=K_{\pi}$ is determined in the graph $G_{\pi}(X)$ by the adjunction relations

$$
\left(K+E, E_{i}\right)=\delta_{i}-2,
$$


where here $\delta_{i}$ is the valency of the vertex $i$ in $G_{\pi}(X)$. Let $L$ be the lattice $H_{2}(\tilde{X}, \mathbb{Z})$ with the intersection form $I(G)$. Then for each vertex $i$ of $G$ one can define $E_{i}^{*} \in L$ with $\left(E_{i}^{*}, E_{j}\right)=-\delta_{i j}$ (the negative of the Kronecker delta), ie the sign-modified dual basis of $L$. (Since $\operatorname{det}(-I(G))=1$, they are well-defined.) Therefore, (2.3.2) reads as $K+E=\sum_{i}\left(2-\delta_{i}\right) E_{i}^{*}$. Since all the valency 2 vertices of $G$ are irrelevant, the relation descends naturally to the level of $\Gamma=\Gamma_{\pi}(X)$ (this means that the multiplicities of $K+E$ along the vertices of $\Gamma$ are those given on the right):

$$
K+E=\sum_{w \in \mathcal{V}}\left(2-\delta_{w}\right) E_{w}^{*} .
$$

Each $E_{w}^{*}$, considered as divisor supported on $E$, together with a noncompact irreducible divisor intersecting $E_{w}$ in a smooth point of $E$, form a $P$-divisor. Hence, we can use (2.2.3) and we obtain that the multiplicity of $K+E$ along any node $v$ of $\Gamma$ (or in the presence of a divisor $F$, along any node of $\left.\Gamma_{\pi}(X, F)\right)$ is

$$
\sum_{w \in \mathcal{V}}\left(2-\delta_{w}\right) \ell_{v w}
$$

In the presence of the divisor $W^{\prime}=\sum_{a \in \mathcal{A}_{W}}\left(i_{a}-1\right) W_{a}^{\prime}$ on $X$, again (2.2.3) then yields

$$
v_{v}=\sum_{w \in \mathcal{V}}\left(2-\delta_{w}\right) \ell_{v w}+\sum_{a \in \mathcal{A}_{W}}\left(i_{a}-1\right) \ell_{v a}
$$

We warn the reader about the following fact. The sum (2.3.4) is associated with $\Gamma_{\pi}(X)$. Therefore, in the applications later, even if we start with some $\Gamma_{\pi}(X, F)$, those ordinary arrowheads associated with $F$ with weight of their supporting edge greater than 1 should be replaced by boundary vertices (hence the summation index $\mathcal{V}$ should be the set of vertices of $\left.\Gamma_{\pi}(X)\right)$. This is valid for the left sum of (2.3.5) too.

2.3.6 Notation In all the next combinatorial formulas associated with a splice diagram $\Gamma_{\pi}(X, F, W)$, the resolution $\pi$ or the geometric source of the diagram is irrelevant.

In particular, in the sequel $\Gamma(F, W)$ means a splice diagram with two $P$-divisors $F$ and $W$. If $W=0$ then we just write $\Gamma(F)$. The divisor $W$ will always be linked with $K$ (determined in $\Gamma$ by Section 2.3.1) in the expression $K+W$.

\subsection{Topological zeta functions of diagrams}

In [27] the second author derived a formula for the topological zeta function of a plane curve germ $f$ in terms of its so-called relative log canonical model; this can be interpreted as being in terms of $\Gamma_{\pi}(X, f)$ (where $\pi$ is minimal). The same proof 
yields a similar formula in our more general context of Section 2.3, with the divisors $F^{\prime}$ and $W^{\prime}$ on the germ $(X, 0)$. In fact one associates in this way a zeta function to a decorated diagram $\Gamma(F, W)$; cf Section 2.3.6. We want to formalize this, since our technique to study the topological zeta function is in fact a "splicing formula" for zeta functions of decorated splice diagrams.

Therefore, let us consider a decorated splice diagram $\Gamma(F, W)$ as in Section 2.3.6. Moreover, it is convenient to associate multiplicities $N_{a}$ and $i_{a}-1$ to all $a \in \mathcal{A}_{F} \cup \mathcal{A}_{W}$, that is, we put $N_{a}=0$ for $a \in \mathcal{A}_{W} \backslash \mathcal{A}_{F}$ and $i_{a}=1$ for $a \in \mathcal{A}_{F} \backslash \mathcal{A}_{W}$.

2.4.1 Definition Let $\Gamma=\Gamma(F, W)$ be such a diagram. We require for each $a \in$ $\mathcal{A}_{F} \cup \mathcal{A}_{W}$ that $\left(N_{a}, i_{a}\right) \neq(0,0)$. For a node $v$, let $\left(\mathcal{A}_{F} \cup \mathcal{A}_{W}\right)_{v}$ and $\mathcal{B}_{v}$ be the (ordinary and/or dashed) arrowheads and boundary vertices, respectively, attached at $v$. Denote the weight at $v$ on the incident edge in the direction of such an arrowhead $a$ or boundary vertex $w$ by $d_{v a}$ and $d_{v w}$, respectively. For any $w \in \mathcal{B}_{v}$, let $i_{w}-1$ be the decoration of the dashed arrowhead supported by $w$; if such an arrowhead does not exist then set $i_{w}=1$. For a special edge $e$, let $v$ and $w$ denote its end vertices, and $q_{e}$ the edge determinant (2.1.2). Then the zeta function $Z(\Gamma)$ of the diagram $\Gamma$ is

$$
\begin{aligned}
Z(\Gamma)=Z(\Gamma ; s):=\sum_{v \in \mathcal{N}} \frac{1}{v_{v}+s N_{v}}\left(2-\delta_{v}^{\prime \prime}\right. & \left.+\sum_{w \in \mathcal{B}_{v}} \frac{d_{v w}}{i_{w}}+\sum_{a \in\left(\mathcal{A}_{F} \cup \mathcal{A}_{W}\right)_{v}} \frac{d_{v a}}{i_{a}+s N_{a}}\right) \\
& +\sum_{e \in \mathcal{E}^{s}} \frac{q_{e}}{\left(v_{v}+s N_{v}\right)\left(v_{w}+s N_{w}\right)} .
\end{aligned}
$$

\section{Splicing the diagrams and their invariants}

\subsection{Splicing the diagrams}

The main advantage of the splice diagrams is that they describe in an ideal way the splice (nonminimal JSJ-) decomposition of the 3-manifold $M$ into its Seifert pieces: while doing this operation, the decorations follow rather simple rules. The behavior of the multiplicities $\left\{N_{w}\right\}_{w}$ associated with a principal divisor $f$ is classical, it was developed in [9].

It is easy to see that any $P$-divisor follows the same formula. On the other hand, the rules for the numbers $\left\{v_{v}\right\}_{v}$ are slightly more involved, and we were not able to find them in the literature (though, see the "simpler" situation considered in [19]). In this subsection, we will present these splice formulae.

Section 3.1.1 treats the case $\Gamma=\Gamma_{\pi}(X)$, Section 3.1.2 the case $\Gamma_{\pi}(X, f)$ and its generalization $\Gamma(F)$, while Section 3.1.5 the case $\Gamma(F, W)$. 
3.1.1 Splicing $\Gamma$ First, recall that splicing along the edge $e$ with end-nodes $v_{L}$ and $v_{R}$ (left/right) is the operation which replaces the left diagram $\Gamma$ into the two diagrams $\Gamma_{L}$ and $\Gamma_{R}$ (containing $v_{L}$ and $v_{R}$ respectively), with two new end vertices $\bar{v}_{L}$ and $\bar{v}_{R}$. (All the other parts of the diagrams are kept unmodified.) If either $d$ or $d^{\prime}$ is 1 , then the corresponding leg in $\Gamma_{L}$ or $\Gamma_{R}$ can be deleted in the minimal representation, but we prefer to keep it. In this way, the valency of the vertices $v_{L}$ and $v_{R}$ stays unmodified. Moreover, when we equip such a leg later with an arrowhead, it cannot be deleted.

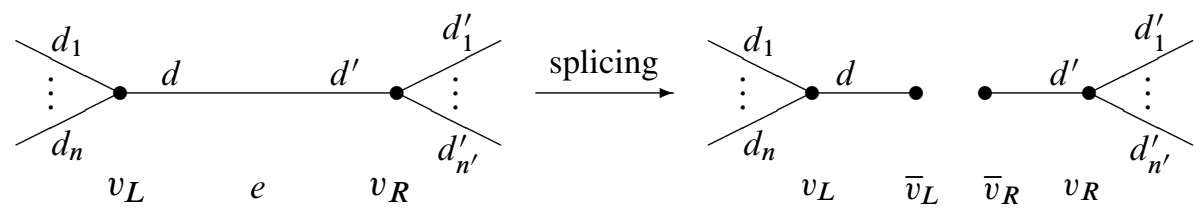

(The diagrams $\Gamma_{L}$ and $\Gamma_{R}$ correspond again to dual graphs of certain IHS normal surface singularities. If $\Gamma$ represents $M=S^{3}$, then both $\Gamma_{L}$ and $\Gamma_{R}$ represent $S^{3}$; see Section 2.1.3.)

3.1.2 Splicing $\Gamma(F)$ Next, we analyze the behavior of the multiplicity system determined by a function $f$ or a $P$-divisor $F$. Let $\mathcal{A}:=\mathcal{A}_{F}$ be the index set of arrowheads; it can be written as a disjoint union $\mathcal{A}_{L} \cup \mathcal{A}_{R}$, according to the position of the arrowheads. First, assume that both $\mathcal{A}_{L}$ and $\mathcal{A}_{R}$ are nonempty. If $a \in \mathcal{A}_{L}$, then let $\ell_{e a}$ be the product of the edge weights, all of them in $\Gamma_{L}$, that are adjacent to, but not on, the path from $v_{R}$ to $a$. Symmetrically, one defines the integers $\ell_{e a}$ for $a \in \mathcal{A}_{R}$. Then $\Gamma(F)$ has the following splice decomposition:
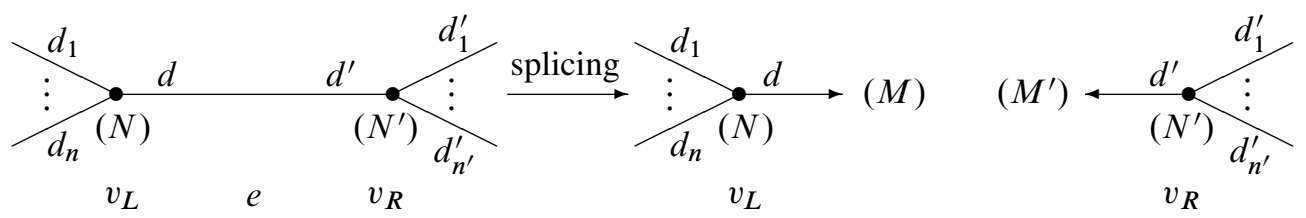

where $(\mathrm{cf}[9,(10.6)])$

$$
M=\sum_{a \in \mathcal{A}_{R}} N_{a} \ell_{e a} \quad \text { and } \quad M^{\prime}=\sum_{a \in \mathcal{A}_{L}} N_{a} \ell_{e a} .
$$

If all the arrowheads of $\Gamma$ are in one side, say $\mathcal{A}_{L}=\varnothing$, then one has the new situation, where $M$ is computed by the same formula as in (3.1.3):
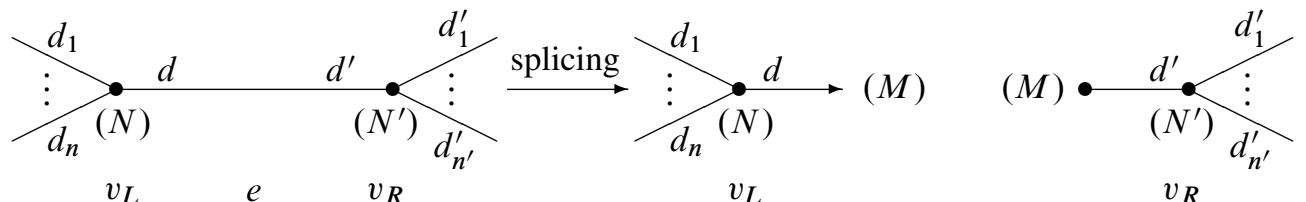
We denote the "total" inherited divisors on $\Gamma_{L}$ and $\Gamma_{R}$ by $F_{L}$ and $F_{R}$, respectively. They can be identified with $P$-divisors of the diagrams $\Gamma_{L}$ and $\Gamma_{R}$, respectively.

3.1.4 Remark When $F=\operatorname{div}(f)$ is a plane curve germ, the left and right graphs above correspond again to dual graphs associated to plane curve germs. In particular, the arrowheads with multiplicities $M$ and $M^{\prime}$ correspond to components of these new germs.

3.1.5 Splicing $\Gamma(F, W)$ Finally, let us analyze the behavior of the divisor of a $2-$ form $\omega$ or, more generally, $K+W$ for some $P$-divisor $W$. Since the splicing of $W$ is covered by the previous step (valid for any $P$-divisor), we have to understand what happens to $K$ only.

Let us consider the splicing of $\Gamma$ along $e$ as in Section 3.1.1. Any invariant associated with $\Gamma$ has its analogue for $\Gamma_{L}$ and $\Gamma_{R}$. We wish to compare the divisors of the pullbacks of the forms $\omega_{0, \Gamma}$ with those of $\omega_{0, \Gamma_{L}}$ and $\omega_{0, \Gamma_{R}}$ - if they exist analytically; and, more generally $K_{\Gamma}$ with $K_{\Gamma_{L}}$ and $K_{\Gamma_{R}}$ (a combinatorial, always well-posed question). For any $v \in \mathcal{V}\left(\Gamma_{L}\right)$, we denote the dual basis element computed in $\Gamma_{L}$ by $E_{v, \Gamma_{L}}^{*}$. Moreover, we separate the vertices of $\Gamma_{L}$ inherited from $\Gamma$ : we set $\overline{\mathcal{V}}\left(\Gamma_{L}\right):=\mathcal{V}\left(\Gamma_{L}\right) \backslash\left\{\bar{v}_{L}\right\}$ (cf the notation of Section 3.1.1), and similarly for $\Gamma_{R}$. Let us rewrite (2.3.3) into

$$
(K+E)_{\Gamma}=\sum_{v \in \overline{\mathcal{V}}\left(\Gamma_{L}\right)}\left(2-\delta_{v}\right) E_{v}^{*}+\sum_{v \in \overline{\mathcal{V}}\left(\Gamma_{R}\right)}\left(2-\delta_{v}\right) E_{v}^{*} .
$$

Recall that $E_{v}^{*}$ behaves as a $P$-divisor associated with one arrowhead supported on $v$. So, by (2.2.3), for any $v \in \overline{\mathcal{V}}\left(\Gamma_{L}\right)$ the restrictions satisfy $\left.E_{v}^{*}\right|_{\overline{\mathcal{V}}\left(\Gamma_{L}\right)}=\left.E_{v, \Gamma_{L}}^{*}\right|_{\overline{\mathcal{V}}\left(\Gamma_{L}\right)}$ (ie, the multiplicities along $\overline{\mathcal{V}}\left(\Gamma_{L}\right)$ agree). Hence

$$
\left.(K+E)_{\Gamma}\right|_{\overline{\mathcal{V}}\left(\Gamma_{L}\right)}=\left.\left((K+E)_{\Gamma_{L}}-E_{\bar{v}_{L}, \Gamma_{L}}^{*}+\sum_{v \in \overline{\mathcal{V}}\left(\Gamma_{R}\right)}\left(2-\delta_{v}\right) E_{v}^{*}\right)\right|_{\overline{\mathcal{V}}\left(\Gamma_{L}\right)} .
$$

Clearly, all multiplicities of both $E_{\Gamma}$ and $E_{\Gamma_{L}}$ along $\overline{\mathcal{V}}\left(\Gamma_{L}\right)$ are one, hence they cancel:

$$
\left.K_{\Gamma}\right|_{\overline{\mathcal{V}}\left(\Gamma_{L}\right)}=\left.\left(K_{\Gamma_{L}}-E_{\bar{v}_{L}, \Gamma_{L}}^{*}+\sum_{v \in \overline{\mathcal{V}}\left(\Gamma_{R}\right)}\left(2-\delta_{v}\right) E_{v}^{*}\right)\right|_{\overline{\mathcal{V}}\left(\Gamma_{L}\right)} .
$$

Using again (2.2.3), the sum can be replaced by a $P$-divisor of $\Gamma_{L}$. Indeed, set

$$
i=i_{e, L}:=\sum_{v \in \overline{\mathcal{V}}\left(\Gamma_{R}\right)}\left(2-\delta_{v}\right) \ell_{e v}
$$


where $\ell_{e v}$ (for any $v \in \overline{\mathcal{V}}\left(\Gamma_{R}\right)$ ) is the product of the edge weights of $\Gamma$, all of them in $\Gamma_{R}$, that are adjacent to, but not on, the path from $v_{L}$ to $v$. Furthermore, let $G_{L}$ be the $P$-divisor on $\Gamma_{L}$ determined by one arrowhead with multiplicity one supported on $\bar{v}_{L}$. Then (2.2.3) and (3.1.7) imply

$$
\left.K_{\Gamma}\right|_{\overline{\mathcal{V}}\left(\Gamma_{L}\right)}=\left.\left(K_{\Gamma_{L}}+(i-1) G_{L}\right)\right|_{\overline{\mathcal{V}}\left(\Gamma_{L}\right)} .
$$

If the forms above exist, and if $G_{L}$ is the pullback divisor of a function $g_{L}$, then

$$
\left.\operatorname{div}\left(\pi^{*} \omega_{0, \Gamma}\right)\right|_{\overline{\mathcal{V}}\left(\Gamma_{L}\right)}=\left.\operatorname{div}\left(\pi_{L}^{*}\left(g_{L}^{i-1} \cdot \omega_{0, \Gamma_{L}}\right)\right)\right|_{\overline{\mathcal{V}}\left(\Gamma_{L}\right)} .
$$

Obviously, there is a symmetric identity for the restriction on $\overline{\mathcal{V}}\left(\Gamma_{R}\right)$. On diagrams:
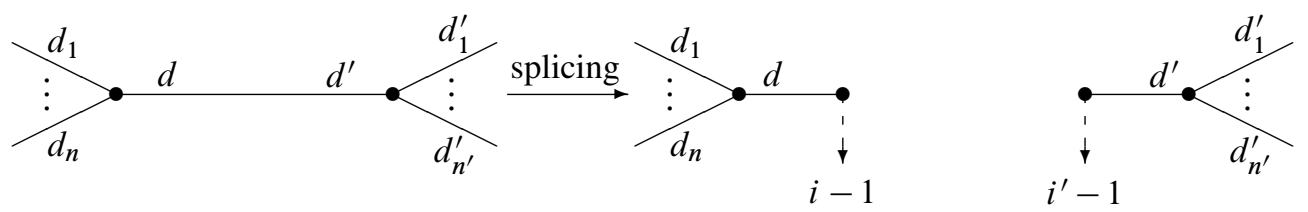

When we incorporate also the divisor $W$, the equations (3.1.8) and (3.1.9) respectively extend to

$$
i:=\sum_{v \in \overline{\mathcal{V}}\left(\Gamma_{R}\right)}\left(2-\delta_{v}\right) \ell_{e v}+\sum_{a \in \mathcal{A}_{W, R}}\left(i_{a}-1\right) \ell_{e a},
$$

where $\ell_{e a}$ is the product of the edge weights of $\Gamma$, all of them in $\Gamma_{R}$, that are adjacent to, but not on, the path from $v_{L}$ to the corresponding dashed arrow in $\Gamma_{R}$, and

$$
\left.\left(K_{\Gamma}+W\right)\right|_{\overline{\mathcal{V}}\left(\Gamma_{L}\right)}=\left.\left(K_{\Gamma_{L}}+(i-1) G_{L}+W_{L}^{+}\right)\right|_{\overline{\mathcal{V}}\left(\Gamma_{L}\right)},
$$

where $W_{L}^{+}$is induced by $W$ on $\Gamma_{L}$, as in Section 3.1.2. We will reserve the notation $W_{L}$ rather for $(i-1) G_{L}+W_{L}^{+}$, in order to have the expression

$$
\left.\left(K_{\Gamma}+W\right)\right|_{\overline{\mathcal{V}}\left(\Gamma_{L}\right)}=\left.\left(K_{\Gamma_{L}}+W_{L}\right)\right|_{\overline{\mathcal{V}}\left(\Gamma_{L}\right)} .
$$

Note that (usually) the vertices $\bar{v}_{L}$ and $\bar{v}_{R}$ have valency 2 (counting all the arrowheads), hence in the formulas considered in the next sections they will be irrelevant.

In the presence of a $P$-divisor $F$, with both $\mathcal{A}_{F, L}$ and $\mathcal{A}_{F, R}$ nonempty, the above diagram modifies into

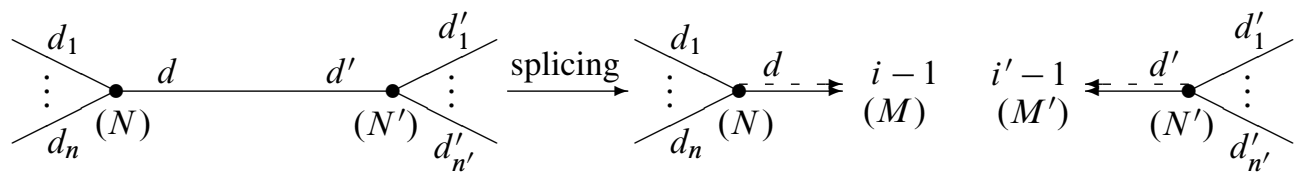

where $M$ and $M^{\prime}$ are determined as in (3.1.3). 
If $\mathcal{A}_{F, L}=\varnothing$, then one has
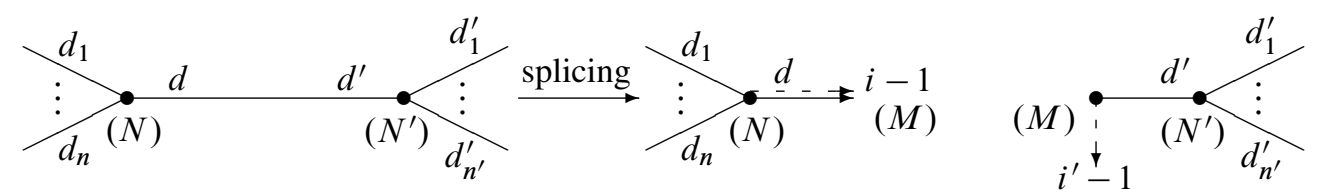

3.1.14 Remark (1) Assume that we start with $W=0$. Then both $W_{L}^{+}$and $W_{R}^{+}$ are zero, but in general $W_{L}$ and $W_{R}$ are not. Furthermore, even if $W$ is effective (in particular, if $W=0$ ), the induced divisors $W_{L}$ and/or $W_{R}$ are in general not effective.

In a different language: even if we start with the "standard 2-from" $\omega_{0}$ (instead of the more general $\left.g \omega_{0}\right)$ - like in the traditional framework of, say, topological or motivic zeta functions - once an inductive splice-decomposition argument is used, we are forced to enlarge the class of our forms: in (3.1.10) the standard form decomposes in the splice component into a generalized form of type $g \omega_{0}$. Also, one can see that even if we start with a holomorphic form, the forms on the splice components, usually, are not holomorphic (that is, they are meromorphic).

(2) Even if we have a precise analytic realization of a diagram $\Gamma$, it is not clear what the relations are connecting this analytic structure and the eventual analytic realizations of $\Gamma_{L}$ and $\Gamma_{R}$. In general, there is no analytic construction known by the authors which would define a natural analytic structure with topology $\Gamma_{L}$ starting from the original $(X, 0)$.

In the presence of functions and forms the situation becomes even more difficult. In that case it might happen that even if we know that $\Gamma(F, W)$ is analytically realized as $\Gamma_{\pi}(X, f, \omega)$, after splicing the two combinatorial packages might not be realized analytically (for example, the "correction term" $(i-1) G_{L}$ is maybe not the divisor of a function).

Nevertheless, if $\Gamma$ represents $S^{3}$, ie if any realization of $\Gamma$ is smooth, then all the functions and (meromorphic) forms will exist.

3.1.15 Example Consider the following diagram $\Gamma(F)$; compare also with Remark 2.2.6(4):

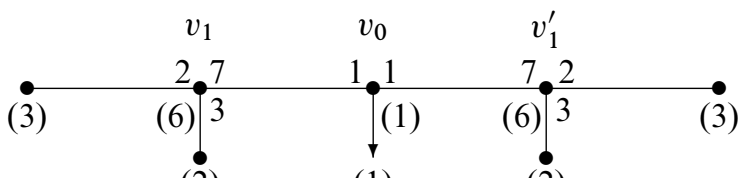

(2) (1)

(2) 
We denote the nodes by $v_{1}, v_{0}$ and $v_{1}^{\prime}$, and their $v$-numbers by $v_{1}, v_{0}$ and $v_{1}^{\prime}$. Then $v_{1}=v_{1}^{\prime}=-13$ and $v_{0}=-2$. Splicing the diagram $\Gamma(F, W=0)$ we get the three star-shaped subgraphs $\Gamma_{1}, \Gamma_{0}$ and $\Gamma_{1}^{\prime}$ :

$$
v_{1}=-13 \quad v_{0}=-2 \quad v_{1}^{\prime}=-13
$$

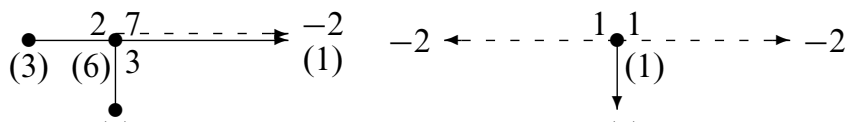

(2)

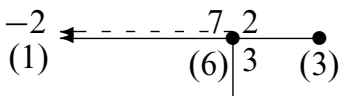

(2)

The zeta function $Z(\Gamma ; s)$ associated with $\Gamma(F, W=0)$ (cf Definition 2.4.1) is

$$
Z(\Gamma ; s)=2 \frac{4}{6 s-13}+\frac{1}{s-2}\left(-1+\frac{1}{s+1}\right)+2 \frac{1}{(s-2)(6 s-13)} .
$$

\subsection{Splicing the topological zeta function}

We will analyze the splicing behavior of the topological zeta function of a graph $\Gamma(F, W)$. Let us consider again the splicing of the diagram $\Gamma(F, W)$ along the edge $e$ as in Section 3.1.5, where we insert the relevant integers $(N, v-1)$ for both vertices $v_{L}$ and $v_{R}$ :
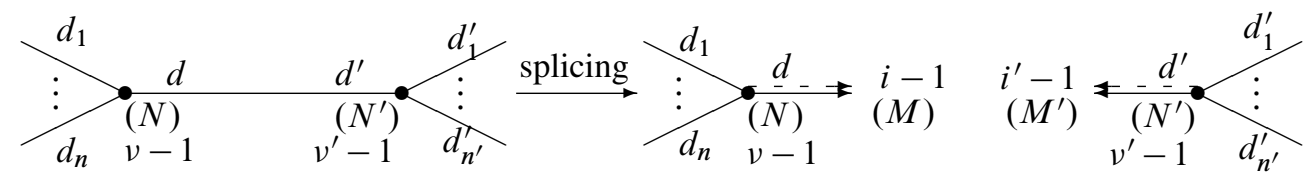

If $\mathcal{A}_{F, L}=\varnothing$, then replace this diagram with the adapted one as in the last diagram of Section 3.1.5. In this case of $\mathcal{A}_{F, L}=\varnothing$ one always has $M^{\prime}=0$.

Note that, to be able to define $Z\left(\Gamma_{L}\right)$ and $Z\left(\Gamma_{R}\right)$, we need that the condition $\left(N_{a}, i_{a}\right) \neq$ $(0,0)$ for all arrowheads is also valid after splicing; see Definition 2.4.1. For the moment we just assume this. In the context of allowed divisors we will show in Lemma 4.2.1 that it is always true.

The contribution of $e$ to $Z(\Gamma)$ turns out to be the sum of the contribution of the "right leg" of $\Gamma_{L}$ to $Z\left(\Gamma_{L}\right)$ and the contribution of the "left leg" of $\Gamma_{R}$ to $Z\left(\Gamma_{R}\right)$, minus an easy correction term, as shown below. This then yields a simple splicing formula for topological zeta functions. We start with the following numerical relation.

3.2.1 Lemma We use the notation of Section 3.1.5, as indicated on the diagram above, and put also $q:=d d^{\prime}-\left(\prod_{j=1}^{n} d_{j}\right)\left(\prod_{j=1}^{n^{\prime}} d_{j}^{\prime}\right)$ for the edge determinant of $e$. If 
$\mathcal{A}_{F, L}=\varnothing$, set $M^{\prime}=0$. Then we have the equality

$$
\begin{gathered}
\frac{q}{(v+s N)\left(v^{\prime}+s N^{\prime}\right)}=\frac{d}{(v+s N)(i+s M)}+\frac{d^{\prime}}{\left(v^{\prime}+s N^{\prime}\right)\left(i^{\prime}+s M^{\prime}\right)} \\
-\frac{1}{(i+s M)\left(i^{\prime}+s M^{\prime}\right)} .
\end{gathered}
$$

Moreover, if two of the pairs $(v, N),\left(v^{\prime}, N^{\prime}\right),(i, M)$ and $\left(i^{\prime}, M^{\prime}\right)$ are linearly dependent, then any other choice of two pairs are also linearly dependent.

Proof The equations (2.2.3) and (3.1.3), respectively (2.3.5) and (3.1.11), imply

$$
\left\{\begin{array} { l } 
{ N = ( \prod _ { j = 1 } ^ { n } d _ { j } ) M + d M ^ { \prime } , } \\
{ N ^ { \prime } = ( \prod _ { j = 1 } ^ { n ^ { \prime } } d _ { j } ^ { \prime } ) M ^ { \prime } + d ^ { \prime } M , }
\end{array} \quad \text { and } \quad \left\{\begin{array}{l}
v=\left(\prod_{j=1}^{n} d_{j}\right) i+d i^{\prime}, \\
v^{\prime}=\left(\prod_{j=1}^{n^{\prime}} d_{j}\right) i^{\prime}+d^{\prime} i .
\end{array}\right.\right.
$$

Hence (as polynomials in $s$ )

$$
\left\{\begin{array}{l}
\left(\prod_{j=1}^{n} d_{j}\right)(i+s M)=v+s N-d\left(i^{\prime}+s M^{\prime}\right), \\
\left(\prod_{j=1}^{n^{\prime}} d_{j}^{\prime}\right)\left(i^{\prime}+s M^{\prime}\right)=v^{\prime}+s N^{\prime}-d^{\prime}(i+s M) .
\end{array}\right.
$$

Multiplying the left and right hand sides of (3.2.3), and using the defining formula of $q$, we obtain

$$
\begin{aligned}
& (v+s N)\left(v^{\prime}+s N^{\prime}\right) \\
& \quad-d\left(v^{\prime}+s N^{\prime}\right)\left(i^{\prime}+s M^{\prime}\right)-d^{\prime}(v+s N)(i+s M)+q(i+s M)\left(i^{\prime}+s M^{\prime}\right)=0 .
\end{aligned}
$$

This is clearly equivalent to (3.2.2). The linear dependency statements follow easily from (3.2.3), using that $q \neq 0$.

One of our main new results here is the next splice decomposition formula for $Z(\Gamma)$.

3.2.4 Theorem (1) Consider the splicing of the diagram $\Gamma$ as in the last diagram of Section 3.1.5. Again, if $\mathcal{A}_{F, L}=\varnothing$, set $M^{\prime}=0$. Then

$$
Z(\Gamma)=Z\left(\Gamma_{L}\right)+Z\left(\Gamma_{R}\right)-\frac{1}{(i+s M)\left(i^{\prime}+s M^{\prime}\right)} .
$$

(2) The contribution of $v_{L}$ in $Z(\Gamma)$ has $-v / N$ as a pole of order 2 if and only if the contribution of $v_{L}$ in $Z\left(\Gamma_{L}\right)$ has $-v / N$ as a pole of order 2 .

(3) Suppose that $-v / N$ is not a pole of order 2 of $Z(\Gamma)$. Then the contributions of $v_{L}$ to the residue of $Z(\Gamma)$ and to the residue of $Z\left(\Gamma_{L}\right)$ at $-v / N$ are exactly the same. 
Proof (1) This is a direct consequence of (3.2.2), since the other contributions to $Z(\Gamma)$ appear in a disjoint way as the other contributions to $Z\left(\Gamma_{L}\right)$ or $Z\left(\Gamma_{R}\right)$.

(2) First note that the coefficient of any expression $1 /(v+s N)^{2}$ in the topological zeta function formula is always positive, hence there are no cancelations among them. Consequently, the statement follows immediately from the linear dependency considerations in Lemma 3.2.1.

(3) The difference between the contributions to both residues is

$$
\frac{1}{N}\left(\frac{q}{v^{\prime}+s N^{\prime}}-\frac{d}{i+s M}\right)
$$

evaluated in $s=-v / N$, and this is zero because of (3.2.2).

3.2.5 Example With the notation of Example 3.1.15 one has

$$
Z\left(\Gamma_{1} ; s\right)=Z\left(\Gamma_{1}^{\prime} ; s\right)=\frac{1}{6 s-13}\left(4+\frac{7}{s-1}\right), \quad Z\left(\Gamma_{0} ; s\right)=\frac{1}{s-2}\left(-3+\frac{1}{s+1}\right) .
$$

One verifies that indeed, according to Theorem 3.2.4(1),

$$
Z(\Gamma ; s)=Z\left(\Gamma_{1} ; s\right)+Z\left(\Gamma_{0} ; s\right)+Z\left(\Gamma_{1}^{\prime} ; s\right)-2 \frac{1}{(-1)(s-1)},
$$

and in the sum $Z(\Gamma ; s)$ the pole $s=1$ "disappears".

\subsection{Splicing the monodromy zeta function and Alexander polynomial}

Let $f:(X, 0) \rightarrow(\mathbb{C}, 0)$ be the germ of a holomorphic function as in Section 2.2, let $F_{0}$ be its Milnor fiber, $h_{i}: H_{i}\left(F_{0}, \mathbb{C}\right) \rightarrow H_{i}\left(F_{0}, \mathbb{C}\right)$ the algebraic monodromy $(i=0,1)$, $\Delta_{i}(t):=\operatorname{det}\left(t I-h_{i}\right)$ the characteristic polynomial of $h_{i}$, and finally, $\zeta(t)=\Delta_{1} / \Delta_{0}$ the monodromy zeta function associated with $f$ at 0 .

It is well-known ( $\mathrm{cf}[9,(11.3)])$ that the zeta function can be computed from the splice diagram $\Gamma=\Gamma_{\pi}(X, f)$ as follows:

$$
\zeta(t)=\prod_{v \in \mathcal{V}(\Gamma)}\left(t^{N_{v}}-1\right)^{\delta_{v}^{\prime}-2},
$$

where, for each vertex $v, N_{v}$ denotes its multiplicity and $\delta_{v}^{\prime}$ its valency in $\Gamma_{\pi}(X, f)$. The zeta-function is "almost" multiplicative with respect to the splice decomposition. In order to have a uniform statement, we consider the Alexander polynomial (in one variable) - cf $[9,(12.1)]$ - as follows:

$$
\Lambda(t):= \begin{cases}\zeta(t) & \text { if } \# \mathcal{A}\left(\Gamma_{\pi}(X, f)\right) \geq 2, \\ \Delta_{1}(t) & \text { if } \# \mathcal{A}\left(\Gamma_{\pi}(X, f)\right)=1 .\end{cases}
$$


The formula (3.3.1) provides $\Lambda(t)$ too, since, if $\# \mathcal{A}=1$, then $\Lambda=\zeta \cdot \Delta_{0}$, and $\Delta_{0}(t)=t^{N_{a}}-1$, where $N_{a}$ is the multiplicity of the unique arrowhead $a$. (In general, $F_{0}$ has $d$ connected components, hence $\Delta_{0}(t)=t^{d}-1$, where $d:=\operatorname{gcd}_{a \in \mathcal{A}}\left(N_{a}\right)$.) In particular, $\Lambda(t)$ can be recovered from the diagram $\Gamma=\Gamma_{\pi}(X, f)$; let us write $\Lambda_{\Gamma}(t)$ for this expression.

Clearly, $\Lambda_{\Gamma}(t)$ depends only on the divisor of $f$, hence the above formula defines $\Lambda_{\Gamma}(t)$ for any $P$-divisor $F$ and $\Gamma(F)$. Moreover, assume that the splice diagram $\Gamma(F)$ has the splice decomposition $\Gamma_{L}$ and $\Gamma_{R}$ as in Section 3.1.2, without considering or asking any analytic realization. Then, analyzing the splice decompositions of Section 3.1.2 and the formula (3.3.1), we easily get the following.

\subsubsection{Proposition $\Lambda_{\Gamma}(t)=\Lambda_{\Gamma_{L}}(t) \cdot \Lambda_{\Gamma_{R}}(t)$.}

3.3.4 Example With the notation of Example 3.1.15 one has $\Lambda_{\Gamma_{1}}(t)=\Lambda_{\Gamma_{1}^{\prime}}(t)=$ $t^{2}-t+1, \Lambda_{\Gamma_{0}}(t)=1$, and their product is indeed $\Lambda(t)=\Delta_{1}(t)=\left(t^{2}-t+1\right)^{2}$.

The advantage (at least in the present paper) of $\Lambda$ compared with $\zeta$ is that $\Lambda$ is a polynomial, hence we do not have to deal with possible cancelations of the roots and poles in the multiplicative formula of Proposition 3.3.3.

\section{Allowed forms/ $\boldsymbol{P}$-divisors}

\subsection{Definition}

In the original setting of a plane curve $f$ we want to pin down a class of 2-forms $\omega$ such that we can realize the goals of Goals 1.2.3 from the introduction. More generally, starting with an effective divisor $F$ on a IHS germ $(X, 0)$, we look for an appropriate class of Weil divisors $W$.

From the point of view of splicing, our definition of allowed forms/divisors below is quite natural. It is not difficult to identify a natural class of divisors $W$ that do the job on a "basic building block", ie a star-shaped graph. We use this as guideline to identify our allowed divisors on a general graph, just demanding that we obtain allowed divisors on all star-shaped subgraphs after (repeated) splicing.

Again, the restriction is combinatorial, depending only on the splicing graph; hence, we will treat allowed $P$-divisors $W$ of graphs $\Gamma(F)$.

4.1.1 Definition Let $\Gamma=\Gamma(F)$ be a diagram as in Section 2.2; see also Section 2.3.6. The set of decorated dashed arrows $\sum_{a \in \mathcal{A}_{W}}\left(i_{a}-1\right) W_{a}$, ie, the associated $P$-divisor $W$, is allowed for $\Gamma$ (or, the diagram $\Gamma(F, W)$ is allowed), if the following conditions are satisfied: 
(1) $i_{a} \neq 0$ for $a \in \mathcal{A}_{W} \backslash \mathcal{A}_{F}$, that is, $\left(N_{a}, i_{a}\right) \neq(0,0)$ for all $a \in \mathcal{A}_{F} \cup \mathcal{A}_{W}$.

(2) Suppose that $\Gamma$ is star-shaped. Let the central node be connected to $n$ boundary vertices whose supporting edges have decorations $\left\{d_{\ell}\right\}_{\ell=1}^{n}$, and with $r$ other incident edges connecting with arrowheads, doubled by dashed arrows or not ( $r \geq 1$ always).

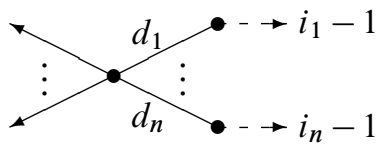

$r$ arrowheads which might be doublearrows

$n$ boundary vertices

Then the decorations $i_{1}-1, \ldots, i_{n}-1$ of the dashed arrows at these boundary vertices are subject to the following restrictions provided that $r=1$ or $r=2$ :

- For $r=1$, if $d_{\ell} \mid i_{\ell}$ for at least $n-1$ indexes $\ell \in\{1, \ldots, n\}$, then $i_{\ell}=d_{\ell}$ for at least $n-1$ indexes $\ell \in\{1, \ldots, n\}$;

- For $r=2$, if $d_{\ell} \mid i_{\ell}$ for all $1 \leq \ell \leq n$, then $i_{\ell}=d_{\ell}$ for all these indexes $\ell$.

(3) For arbitrary $\Gamma$ we require that the induced decorations on each star-shaped subdiagram of $\Gamma$, obtained after repeated splicing as in Section 3.1.5, satisfy the restrictions (2).

4.1.2 Remark (a) In (2) there are thus no conditions on the decorations $i_{a}$ for the arrowheads given by $a \in \mathcal{A}_{F}$, that is, those associated to the other $r$ edges.

(b) The value $i_{\ell}=1$ is possible. It corresponds to no dashed arrow, or formally to a dashed arrow with decoration zero. Also, for the boundary vertices from the right, if $d_{\ell}=1$ for some $\ell$, and the corresponding leg is not represented as above, but with its minimal diagram as in Section 2.3, then the above definition applies for these dashed arrowheads too (with $d_{\ell}=1$ ).

(c) One can formulate the restrictions in (2) simultaneously for all $r$ as follows:

If $d_{\ell} \mid i_{\ell}$ for at least $n+r-2$ indexes $\ell \in\{1, \ldots, n\}$, then $i_{\ell}=d_{\ell}$ for at least $n+r-2$ of the indexes $\ell$. (This assumption is empty if $r \geq 3$.)

(d) We assumed implicitly in (2) that $n \geq 1$. When $n=0$ the conditions are empty.

(e) A priori it is not clear at all that there exist allowed $W$ on a general graph $\Gamma(F)$. We will construct plenty of them later.

4.1.3 Definition Let $(X, 0)$ be an IHS surface germ, and $F^{\prime}$ a (nonzero) effective Weil divisor on it. A Weil divisor $W^{\prime}$ of $(X, 0)$ is allowed for the pair $\left(X, F^{\prime}\right)$ if there exists an embedded resolution $\pi: \tilde{X} \rightarrow X$ of $F^{\prime}$ such that the diagram $\Gamma_{\pi}(X, F, W)$ is allowed. 
This notion is well defined, in the sense that it is invariant under "extra" blowing-ups, as shown below.

4.1.4 Proposition We use the notation from Definition 4.1.3. Suppose that the diagram $\Gamma:=\Gamma_{\pi}(X, F, W)$ is allowed. Let $h: \tilde{X}_{1} \rightarrow \tilde{X}$ be a blowing-up in some point of $\tilde{X}$. Then the diagram $\Gamma_{1}:=\Gamma_{\pi \circ h}\left(X, F_{1}, W_{1}\right)$ (with obvious notation) is also allowed.

Proof We only have to investigate the spliced star-shaped subgraphs of $\Gamma_{1}$ that are new or different with respect to $\Gamma$. If the centre $P$ of the blowing-up $h$ is either a point of a boundary curve, or an intersection point of two components (exceptional or strict transform), we are done because then $\Gamma_{1}=\Gamma$. We are left with the following two cases for $P$.

Case $1 P$ is a point of $E_{j}^{\circ}$ (that is, a generic point of $E_{j}$ ), where the vertex corresponding to $E_{j}$ has valency 2 in $G_{\pi}(X, F, W$ ) (so it does not occur explicitly in $\Gamma$ ):

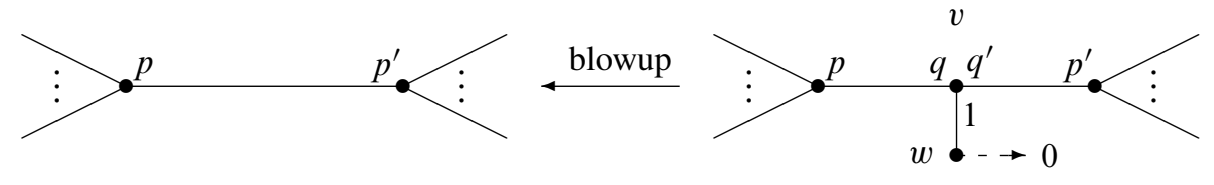

(Above we did not insert the information about $F$; and one of the nodes of the diagram before blowup can be replaced by a boundary vertex with or without dashed arrows.)

It is not difficult to verify that the spliced star-shaped subgraphs around the "old" nodes in $\Gamma$ and $\Gamma_{1}$ are the same. Moreover, the new spliced star-shaped subgraph of $\Gamma_{1}$ around $v$ satisfies the definition of allowedness. Indeed, in both cases $r=1$ or $r=2$, the fact that the decorations associated to the boundary vertex $w$ satisfy $d_{1}=i_{1}=1$ finishes the verification.

Case $2 P$ is a point of $E_{v}^{\circ}$, where $v$ is a node in $\Gamma$.

In this case the only novelty in $\Gamma_{1}$ is an extra edge at the node $v$ supporting a boundary vertex, again with edge decoration $d_{\ell}=1$ and associated number $i_{\ell}=1$. This again does not affect the allowedness condition for the star-shaped subgraph around $v$.

4.1.5 Remark It is possible that a divisor $W^{\prime}$ on $(X, 0)$ is not allowed in the diagram associated with the minimal embedded resolution $\pi$ of $\left(X, F^{\prime}\right)$, but is allowed in some $\Gamma_{\pi}(X, F, W)$ associated with some nonminimal $\pi$. Consider for example the situation

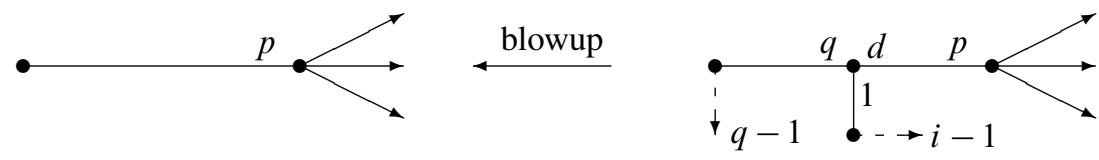


where $\pi$ is obtained from the minimal embedded resolution $\pi_{\min }$ by composing with one blowing-up, and $i \in \mathbb{Z}_{>1}$. The component of the strict transform of $W^{\prime}$ with multiplicity $i-1$ intersects the exceptional divisor of $\pi_{\min }$ in a component $E_{j}$ of valency 2, and this was not permitted; cf Section 2.3.

4.1.6 Remark Assume that $F$ has only one arrowhead with multiplicity 1 . Then for any $W$, the dashed arrowhead with multiplicity $i-1$ which doubles the arrowhead of $F$ has an almost irrelevant geometric contribution. Indeed, its only effect is the following: in any ratio $v_{v} / N_{v}$ it has a global integral $(i-1)$-shift. In particular, in such a situation (having connections with monodromy in mind), we might take $i-1=0$ without restricting the generality of the discussion.

4.1.7 Example Let us continue the discussion of Example 3.1.15. Having in mind Remark 4.1.6, the general form $W$ will have the following dashed arrowheads:

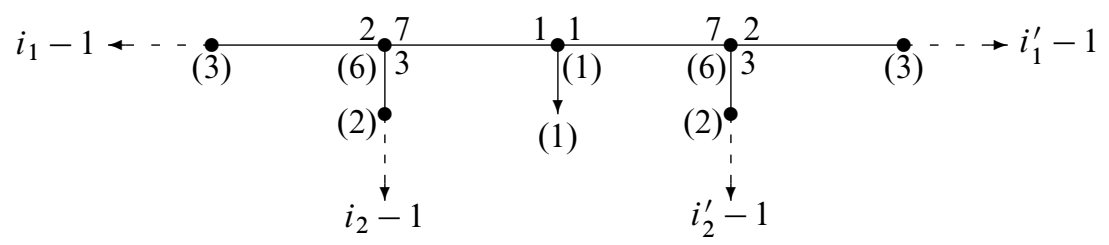

The splice decomposition provides:

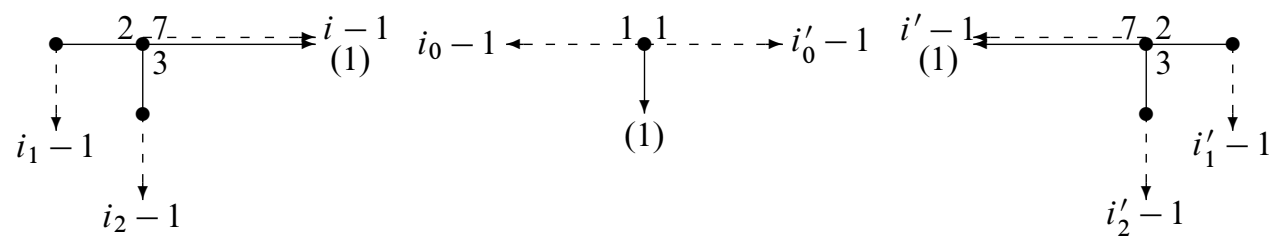

In the above picture $i-1=i_{0}^{\prime}-1=-2+3\left(i_{1}^{\prime}-1\right)+2\left(i_{2}^{\prime}-1\right)$ and $i^{\prime}-1=i_{0}-1=$ $-2+3\left(i_{1}-1\right)+2\left(i_{2}-1\right)$.

Assume that $W$ is allowed. This imposes the following numerical conditions.

- In the middle graph $\Gamma_{0}$ we impose: either $3 i_{1}+2 i_{2}=7$ or $3 i_{1}^{\prime}+2 i_{2}^{\prime}=7$.

- In $\Gamma_{1}$ one gets: if $2 \mid i_{1}$ or $3 \mid i_{2}$ then either $2=i_{1}$ or $3=i_{2}$. Note that if $3 i_{1}+2 i_{2}=7$ then $2 \nmid i_{1}$ and $3 \nmid i_{2}$. There is a symmetric restriction in $\Gamma_{1}^{\prime}$ too: if $2 \mid i_{1}^{\prime}$ or $3 \mid i_{2}^{\prime}$ then either $2=i_{1}^{\prime}$ or $3=i_{2}^{\prime}$.

In particular, the zero form $W=0$ is not allowed. 


\subsection{Restricting and extending allowed divisors}

Consider the splicing of a given diagram $\Gamma(F)$ along a special edge $e$ as in Section 3.1.2. A basic idea in the definition of an allowed $W$ for $\Gamma(F)$ is that the induced $W_{L}$ and $W_{R}$ should be allowed for $\Gamma_{L}\left(F_{L}\right)$ and $\Gamma_{R}\left(F_{R}\right)$, respectively. This is almost clear from the nature of the definitions.

There is potentially a problem when (say) $\mathcal{A}_{F, L}=\varnothing$, since then a new dashed arrowhead at a boundary vertex of $\Gamma_{R}\left(F_{R}\right)$ is created, and it could have associated decoration $i^{\prime}=0$; see the last picture in Section 3.1.5. Indeed, in Definition 4.1.1(3) we asked for each star-shaped subdiagram to satisfy condition (2), but we didn't ask (1). In the next lemma we will verify that (1) will be automatically satisfied.

4.2.1 Lemma When $\mathcal{A}_{F, L}=\varnothing$ and $i_{a} \neq 0$ for all $a \in \mathcal{A}_{W, L}$, then $i^{\prime} \neq 0$. In particular, allowed divisors on a graph always "restrict" to allowed divisors on spliced subdiagrams.

Proof By induction on the number of nodes in $\Gamma_{L}$ it is sufficient to prove that $i^{\prime} \neq 0$ when $\Gamma_{L}$ is star-shaped. Let $i_{1}-1, \ldots, i_{n}-1$ be the multiplicities of the dashed arrowheads along the edges with decorations $d_{1}, \ldots, d_{n}$. Denote $D:=\prod_{\ell=1}^{n} d_{\ell}$. Then, using (3.1.11),

$$
i^{\prime}=(1-n) D+\sum_{\ell=1}^{n} \frac{D}{d_{\ell}} i_{\ell} .
$$

Suppose that $i^{\prime}=0$. Then $d_{\ell}$ divides $\left(D / d_{\ell}\right) i_{\ell}$, hence divides $i_{\ell}$ too, for all $\ell$. By the definition of allowedness we then know that $i_{\ell}=d_{\ell}$ for at least $n-1$ of the $i_{\ell}$; say for $i_{2}, \ldots, i_{n}$. Thus

$$
0=i^{\prime}=(1-n) D+(n-1) D+D i_{1} / d_{1}
$$

and hence $i_{1}=0$, contradicting the assumptions. For the second statement, note that $i_{a}=1$ for any $a \in \mathcal{A}_{F} \backslash \mathcal{A}_{W}$, while $i_{a} \neq 0$ for $a \in \mathcal{A}_{W} \backslash \mathcal{A}_{F}$ since $W$ is allowed.

A crucial question in our setting is the converse: can we "extend" an allowed $W^{\text {b }}$ on $\Gamma_{R}\left(F_{R}\right)$ to $\Gamma(F)$, that is, can we construct an allowed $W$ on $\Gamma(F)$ for which $W_{R}=W^{\mathrm{b}}$ ?

It is enough to study this question when $\Gamma_{L}\left(F_{L}\right)$ is star-shaped, since we can then proceed further inductively.

4.2.2 Proposition Let $\Gamma(F)$ be an arbitrary diagram as in Section 2.2. Splice $\Gamma(F)$ along a special edge $e$ such that $\Gamma_{L}\left(F_{L}\right)$ is star-shaped. Then the map

$$
\Psi:\{\text { allowed } W \text { for } \Gamma(F)\} \rightarrow\left\{\text { allowed } W^{b} \text { for } \Gamma_{R}\left(F_{R}\right)\right\}, \quad W \mapsto W_{R},
$$


is surjective if there is at least one arrowhead in $\Gamma(F)$ on the right of $e$, or the number of arrowheads on the left of $e$ is different from one and two.

Proof Take an allowed $W^{\mathrm{b}}$ for $\Gamma_{R}\left(F_{R}\right)$. Let $i^{\prime}-1$ be the decoration of the dashed arrowhead at the left of $v_{R}$.

Let $i_{1}-1, \ldots, i_{n}-1(n \geq 2)$ be the (still to be determined) decorations of the dashed arrowheads of some $W$, that we want to construct in the preimage of $W^{b}$ by $\Psi$. Let $j-1$ be the decoration of the new induced dashed arrowhead at $v_{L}$ for $\Gamma_{L}\left(F_{L}\right)$. Note that $j$ is fixed in the sense that it is uniquely determined in terms of $W^{b}$ by the formula (3.1.11). (And, when there are no arrowheads on the right of $e$, we have that $j \neq 0$ by the argument in the proof of Lemma 4.2.1.)

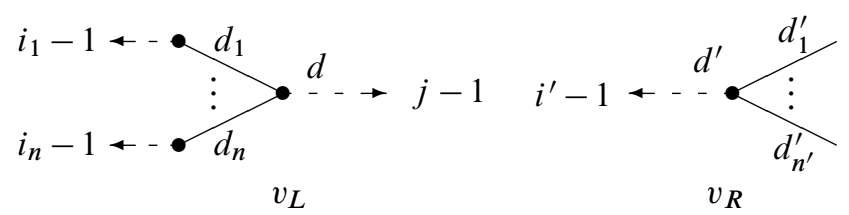

(In this diagram we did not insert the information regarding the divisor $F$, the corresponding arrowhead positions might determine different cases; see below.)

Denote $D:=\prod_{\ell=1}^{n} d_{\ell}$. Then $(\operatorname{cf}(3.1 .11))$ we are searching for $i_{1}, \ldots, i_{n}$ with

$$
i^{\prime}=(1-n) D+\sum_{\ell=1}^{n} \frac{D}{d_{\ell}} i_{\ell} .
$$

Since $\operatorname{gcd}_{\ell}\left\{D / d_{\ell}\right\}=1$, we know that there exist $i_{1}, \ldots, i_{n} \in \mathbb{Z}$ satisfying (4.2.3). We have to verify that this can be done compatibly with the restrictions on the $i_{\ell}$ and $j$, when exactly one or two (ordinary) arrowheads are among the legs in $\Gamma_{L}\left(F_{L}\right)$.

Note first that, by (4.2.3), we have for any $\ell=1, \ldots, n$ that $d_{\ell} \mid i^{\prime}$ if and only if $d_{\ell} \mid i_{\ell}$. With the given assumptions on the arrowheads in $\Gamma(F)$, we encounter two cases.

(1) There is exactly one arrowhead on $\Gamma_{L}\left(F_{L}\right)$, and it coincides with the dashed $(j-1)$-arrowhead. Then we suppose that $d_{\ell} \mid i^{\prime}$ for at least $n-1$ of the $i_{\ell}$, say for $i_{1}, \ldots, i_{n-1}$. (Otherwise nothing has to be verified.)

(2) There are exactly two arrowheads on $\Gamma_{L}\left(F_{L}\right)$, and they coincide with the dashed $(j-1)-$ and $\left(i_{n}-1\right)$-arrowheads. Then we suppose that $d_{\ell} \mid i^{\prime}$ for $\ell=1, \ldots, n-1$.

In each of these cases we take $i_{\ell}=d_{\ell}$ for $\ell=1, \ldots, n-1$ and then, in order to satisfy (4.2.3), we take $i_{n}$ given by $i^{\prime}=D i_{n} / d_{n}$. This way we thus constructed an allowed $W$ for $\Gamma(F)$ that "restricts" to $W^{b}$. 
4.2.4 Remark (a) Consider in the proof above the excluded cases. First assume that there are exactly two arrowheads on $\Gamma_{L}\left(F_{L}\right)$, namely when they coincide with the dashed $\left(i_{n-1}-1\right)-$ and $\left(i_{n}-1\right)$-arrowheads. To verify allowedness we have to suppose that $d \mid j$ and $d_{\ell} \mid i^{\prime}$ for $\ell=1, \ldots, n-2$. Then, in order to get an allowed extension we should have that $d=j$, but this is not true in general.

Similarly, assume that $\Gamma_{L}\left(F_{L}\right)$ has exactly one arrowhead which coincides with the dashed $\left(i_{n}-1\right)$-arrowhead. Then in the situation $d\left|j, d_{\ell}\right| i^{\prime}$ for $\ell \leq n-2$ but $d_{n-1} \nmid i^{\prime}$ one gets an allowed extension only if $d=j$.

These cases motivate the restrictions of Proposition 4.2.2.

This discussion shows the following addendum to Proposition 4.2.2:

4.2.5 Addendum With the above notation, in the following cases an extension is still possible:

(i) either $d \nmid j$ or $d=j$;

(ii) the only arrowhead coincides with the dashed $\left(i_{n}-1\right)$-arrowhead and $d_{\ell} \mid i^{\prime}$ for $\ell \leq n-1$.

(b) Let $\Gamma(F)$ be an arbitrary diagram. Let $\Gamma_{\mathcal{A}}$ be that minimal connected subdiagram of $\Gamma$ which contains those nodes which either support at least one arrowhead of $F$, or sit on a (geodesic) path connecting two arrowheads of $F$, and those boundary vertices which are supported by these nodes. The connected components of $\Gamma \backslash \Gamma_{\mathcal{A}}$ are denoted by $\left\{\Gamma_{j}\right\}_{j \in \mathcal{J}}$, and each $\Gamma_{j}$ is connected to $\Gamma_{\mathcal{A}}$ at the vertex $v_{j}$ of $\Gamma_{\mathcal{A}}$. (For example, if $\Gamma$ is the minimal diagram of a plane curve singularity, then $|\mathcal{J}| \leq 1$.) Then one has the following facts.

- Any allowed $P$-divisor supported on a star-shaped subdiagram centered at any node of $\Gamma_{\mathcal{A}}$ can be extended by Proposition 4.2.2 to an allowed $P$-divisor of the whole $\Gamma(F)$. In particular,

any $\Gamma(F)$ always admits allowed divisors $W$.

- Any allowed $P$-divisor on a star-shaped diagram centered at a vertex $v$ in $\Gamma_{j}$ can be extended "away from $v_{j}$ ". In order to extend it "in the direction of $v_{j}$ " one needs some extra conditions (like in Addendum 4.2.5).

\section{Allowed forms/divisors induce eigenvalues}

\subsection{Poles give eigenvalues}

In this section we prove that the poles of the topological zeta function associated to any $\Gamma(F)$ and allowed divisor $W$ provide eigenvalues for the monodromy zeta function. 
5.1.1 Lemma Let $S$ be the star-shaped diagram as in Definition 4.1.1 with $r=1$ or $r=2$ ordinary arrowheads, equipped with decorations as below. We assume that $i_{\ell}=d_{\ell}$ for $\ell=1, \ldots, n-1$ (and $i_{n} \neq 0$ ) if $r=1$, and $i_{\ell}=d_{\ell}$ for $\ell=1, \ldots, n$ if $r=2$. Thus $W$ is allowed. When $r=2$, we assume also that $-v_{v} / N_{v}$ is not a pole of order 2 of $Z(S)$, that is, $v_{v} / N_{v} \neq k_{1} / N_{1}$ and $v_{v} / N_{v} \neq k_{2} / N_{2}$. Then, in all the above situations, $-v_{v} / N_{v}$ is not a pole of $Z(S)$.

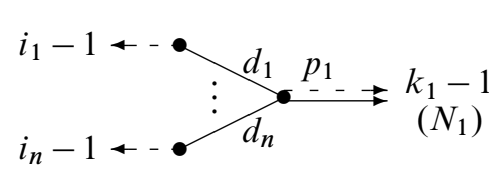

$v$

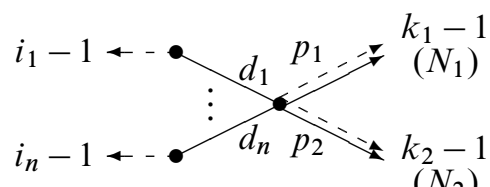

$v$

Proof We consider first the case $r=2$. By (2.3.5) and (2.2.3) we have, with $D=$ $\prod_{\ell} d_{\ell}$,

$$
\begin{aligned}
v_{v} & =\sum_{\ell=1}^{n} \frac{D p_{1} p_{2}}{d_{\ell}} i_{\ell}-n D p_{1} p_{2}+D p_{2} k_{1}+D p_{1} k_{2}, \\
N_{v} & =D\left(p_{2} N_{1}+p_{1} N_{2}\right),
\end{aligned}
$$

respectively. With our assumptions this simplifies to

$$
\begin{aligned}
v_{v} & =D\left(p_{2} k_{1}+p_{1} k_{2}\right), \\
N_{v} & =D\left(p_{2} N_{1}+p_{1} N_{2}\right) .
\end{aligned}
$$

The residue of $-v_{v} / N_{v}$ is (up to a factor $N_{v}$ )

$$
\begin{aligned}
\left.\left(-n+\sum_{\ell=1}^{n} \frac{d_{\ell}}{i_{\ell}}+\frac{p_{1}}{k_{1}+s N_{1}}+\frac{p_{2}}{k_{2}+s N_{2}}\right)\right|_{\left(s=-v_{v} / N_{v}\right)} & \\
& =\frac{p_{1}}{k_{1}-\left(v_{v} / N_{v}\right) N_{1}}+\frac{p_{2}}{k_{2}-\left(v_{v} / N_{v}\right) N_{2}} .
\end{aligned}
$$

This expression being zero is equivalent to

$$
\frac{v_{v}}{N_{v}}=\frac{p_{2} k_{1}+p_{1} k_{2}}{p_{2} N_{1}+p_{1} N_{2}},
$$

which follows from (5.1.3).

When $r=1$, we have by (2.3.5) and (2.2.3) that

$$
v_{v}=\sum_{\ell=1}^{n} \frac{D p_{1}}{d_{\ell}} i_{\ell}-(n-1) D p_{1}+D k_{1} \quad \text { and } \quad N_{v}=D N_{1} \text {, }
$$


simplifying with our assumptions to

$$
v_{v}=D\left(\frac{p_{1}}{d_{n}} i_{n}+k_{1}\right) \text { and } N_{v}=D N_{1} .
$$

Thus $v_{v} / N_{v} \neq k_{1} / N_{1}$ (since $i_{n} \neq 0$ ) and $-v_{v} / N_{v}$ is not a pole of order 2 . The fact that its residue is zero is a similar easy computation as above.

5.1.6 Theorem Let $(X, 0)$ be an IHS germ, and $f$ an analytic function on $X$. Let $W$ be an arbitrary allowed divisor for $(X, f)$. If $s_{0}$ is a pole of the topological zeta function $Z(f, W ; s)$, then $\exp \left(2 \pi i s_{0}\right)$ is a monodromy eigenvalue of $f$ at some point of $\{f=0\}$ (in one of the homology groups).

Proof Fix an embedded resolution $\pi$ of $f$ such that $W$ is allowed for it. We use the usual notation associated to $\Gamma:=\Gamma_{\pi}(X, f, W)$. We consider three subcases.

(1) There is a component $F_{a}$ of $\{f=0\}$ such that $s_{0}=-i_{a} / N_{a}$. Then $\exp \left(2 \pi i s_{0}\right)$ is an eigenvalue of $f$ at a point of $F_{a}$ close to 0 (since all $N_{a}$-th roots of unity are eigenvalues at such a point).

(2) There is no $F_{a}$ as in (1) and let $s_{0}$ be a pole of order 1 of $Z(f, W ; s)$. Then $s_{0}=-v_{v} / N_{v}$ for some node $v$, such that the contribution of $v$ to the residue of $Z(f, W ; s)$ at $s_{0}$ is nonzero. Consider after repeated splicing the induced star-shaped diagram $S$ around $v$; by Theorem 3.2.4 we know that the residue of $Z(S)$ at $s_{0}$ is exactly this contribution.

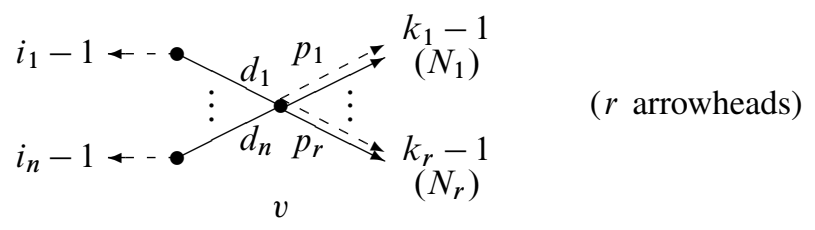

From Section 3.3 we compute

$$
\zeta_{S}(t)=\frac{\left(t^{N_{v}}-1\right)^{r+n-2}}{\prod_{\ell=1}^{n}\left(t^{N_{v} / d_{\ell}}-1\right)},
$$

and by (2.3.5), denoting $D:=\prod_{\ell=1}^{n} d_{\ell}$, we have

$$
v_{v}=\sum_{\ell=1}^{n} \frac{D}{d_{\ell}}\left(\prod_{j=1}^{r} p_{j}\right) i_{\ell}+D \cdot \text { (some integer). }
$$

We distinguish three possibilities for $r$ in order to show that $\exp \left(2 \pi i s_{0}\right)$ is always a root of $\Lambda_{S}(t)$. 
- If $r \geq 3$, then, via (5.1.7), $\exp \left(2 \pi i s_{0}\right)$ is clearly a root of $\Lambda_{S}(t)=\zeta_{S}(t)$.

- If $r=2$, suppose that $\exp \left(2 \pi i s_{0}\right)$ is not a root of $\Lambda_{S}(t)=\zeta_{S}(t)$. Then, by (5.1.7), $v_{v} / d_{\ell}$ must be an integer for all $\ell=1, \ldots, n$. By (5.1.8) this is equivalent to $d_{\ell} \mid i_{\ell}$ for all these $\ell$. By allowedness we conclude that then $i_{\ell}=d_{\ell}$ for all $\ell$. Lemma 5.1.1 then contradicts that the residue at $s_{0}$ is nonzero.

- If $r=1$, suppose that $\exp \left(2 \pi i s_{0}\right)$ is not a root of $\Lambda_{S}(t)=\Delta_{S}(t)$. Then, analogously, for at least $n-1$ of the numbers $i_{1}, \ldots, i_{n}$ we have that $\frac{v_{v}}{d_{\ell}}$ must be an integer, or, equivalently, $d_{\ell} \mid i_{\ell}$. Then by allowedness $i_{\ell}=d_{\ell}$ for $n-1$ of these numbers, and again Lemma 5.1.1 contradicts that the residue at $s_{0}$ is nonzero.

We conclude that $\exp \left(2 \pi i s_{0}\right)$ is indeed always a root of $\Lambda_{S}(t)$, hence of $\Lambda_{\Gamma}(t)$, and thus that $\exp \left(2 \pi i s_{0}\right)$ is a monodromy eigenvalue of $f$ at 0 .

(3) There is no $F_{a}$ as in (1) and let $s_{0}$ be a pole of order 2. This implies that $s_{0}=-v_{v} / N_{v}=-v_{w} / N_{w}$ for two nodes $v$ and $w$, connected by a special edge $e$. Equivalently (cf Theorem 3.2.4),

$$
s_{0}=-\frac{v_{v}}{N_{v}}=-\frac{k}{N}
$$

for the central node $v$ and an arrowhead with decorations $\left(N_{a}, i_{a}\right)=(N, k)$ in some star-shaped spliced subdiagram of $\Gamma$.

Let $C$ be the connected part of $\Gamma$ containing $v$ and consisting of nodes $w$ with $s_{0}=-v_{w} / N_{w}$ only. Either at least one node $w$ in $C$ has at least three attached (ordinary) arrowheads in the induced star-shaped subgraph $S_{w}$ after splicing, and then $\exp \left(2 \pi i s_{0}\right)$ is a root of $\Lambda_{S_{w}}(t)=\zeta_{S_{w}}(t)$; or all nodes $w$ in $C$ have exactly one or two attached arrowheads in $S_{w}$. So we are left with this second possibility. We distinguish two subcases for such a node $v$. For the two diagrams and notation; see just before the proof of Lemma 5.1.1.

- For $r=1$, using (2.2.3) and (2.3.5) (see also (5.1.4)), we have from (5.1.9) that

$$
\frac{v_{v}}{N_{v}}=\frac{\sum_{\ell=1}^{n}\left(D p_{1} / d_{\ell}\right) i_{\ell}-(n-1) D p_{1}+D k_{1}}{D N_{1}}=\frac{k_{1}}{N_{1}} \text {. }
$$

This simplifies to $\sum_{\ell=1}^{n}\left(D / d_{\ell}\right) i_{\ell}-(n-1) D=0$, implying that $d_{\ell} \mid i_{\ell}$ for all $\ell=1, \ldots, n$. By allowedness we then have $i_{\ell}=d_{\ell}$ for say $\ell=2, \ldots, n$. Hence the previous equality reduces to $i_{1}=0$, contradicting that $W$ is allowed. Hence this case cannot occur. 
- For $r=2$, we may assume also that $v$ is an "extremity" of $C$, that is, that $v$ is only connected to one other node of $C$, say $v_{v} / N_{v}=k_{1} / N_{1} \neq k_{2} / N_{2}$. Now (2.2.3) and (2.3.5) (see also (5.1.2)) together with (5.1.9) yield

$$
\frac{v_{v}}{N_{v}}=\frac{\sum_{\ell=1}^{n}\left(D p_{1} p_{2} / d_{\ell}\right) i_{\ell}-n D p_{1} p_{2}+D p_{2} k_{1}+D p_{1} k_{2}}{D\left(p_{2} N_{1}+p_{1} N_{2}\right)}=\frac{k_{1}}{N_{1}} .
$$

Suppose that $\exp \left(2 \pi i s_{0}\right)$ is not a root of $\Lambda_{S_{v}}(t)$. By the same arguments as in case (2) we get that $d_{\ell} \mid i_{\ell}$ for all $\ell=1, \ldots, n$. By allowedness we now have that $i_{\ell}=d_{\ell}$ for all $\ell$, and then the previous equality reduces to

$$
\frac{p_{2} k_{1}+p_{1} k_{2}}{p_{2} N_{1}+p_{1} N_{2}}=\frac{k_{1}}{N_{1}} \text {. }
$$

This is equivalent to $k_{1} / N_{1}=k_{2} / N_{2}\left(=-s_{0}\right)$, contradicting that $v$ is an extremity of $C$.

Thus $\exp \left(2 \pi i s_{0}\right)$ is a root of $\Lambda_{S_{v}}(t)$ for some node $v$, hence also of $\Lambda_{\Gamma}(t)$.

5.1.10 Remark Although we formulated the previous Theorem 5.1.6 for an analytic function $f$, it has a purely combinatorial version (with the same proof) valid for diagrams.

Start with a diagram $\Gamma(F)$ and set the possible "eigenvalues of the monodromies at different points and in different homologies" to be

$$
\text { Eig }:=\left\{\lambda: \Delta_{1}(\lambda)=0\right\} \cup \bigcup_{a \in \mathcal{A}_{F}}\left\{\lambda: \lambda^{N_{a}}=1\right\} .
$$

Then, for any allowed $W$ and pole $s_{0}$ of the zeta function $Z(F, W ; s)$, we have that $\exp \left(2 \pi i s_{0}\right)$ belongs to Eig.

5.1.11 Example Let us continue the main Example 4.1.7 further.

Using Example 3.3.4 we get that the eigenvalues are the roots of $(t-1)\left(t^{2}-t+1\right)$. Theorem 5.1.6 says that if $W$ is allowed and $s_{0}$ is a pole of $Z(s)$, then $\exp \left(2 \pi i s_{0}\right)$ is 1 or a primitive $6-$ th root of unity.

(a) First we show that we can find easily nonallowed forms $W$ such that the corresponding pole will not provide an eigenvalue. This proves that some kind of restriction regarding the divisors $W$ is necessary.

Consider in Example 3.1.15 a general form $W$, not necessarily allowed. Then $v_{1}=$ $-13+21\left(i_{1}-1\right)+14\left(i_{2}-1\right)+18\left(i_{1}^{\prime}-1\right)+12\left(i_{2}^{\prime}-1\right)$, which is congruent with $3 i_{1}+2 i_{2}$ modulo $N_{v_{1}}=6$. We wish to get, for example, $v_{1} \equiv 3(\bmod 6)$, hence we 
might take $i_{1}=1$ and $i_{2}=6$. Assume also that $i_{1}^{\prime}=i_{2}^{\prime}=1$. Then $v_{1}=57, v_{0}=8$ and $v_{1}^{\prime}=47$. Then computing the zeta function we realize that $s_{0}=-57 / 6$ is a pole, but $\exp \left(2 \pi i s_{0}\right)=-1 \notin$ Eig.

(b) It is not hard to find divisors $W$ which are not allowed, but such that nevertheless their poles provide only eigenvalues. Take for example the trivial form $W=0$. Then by Example 4.1.7 it is not allowed, but using the expression for $Z(s)$ from Example 3.1.15 we can conclude that the poles provide eigenvalues.

(c) In order to emphasize the subtlety of the statement of Theorem 5.1.6 (and of its proof) we "will try to find a counterexample" of this fact. Namely, let us take $i_{1}=1$, $i_{2}=3$ and write $I^{\prime}$ for the expression $3 i_{1}^{\prime}+2 i_{2}^{\prime}$. Note that $W$ is allowed exactly when $I^{\prime}=7$; cf Example 4.1.7.

By a computation $v_{1}=6 I^{\prime}-15, v_{0}=I^{\prime}-3$ and $v_{1}^{\prime}=7 I^{\prime}-24$. Moreover, the zeta function $Z(s)$ is

$$
\begin{aligned}
& \frac{2}{6 I^{\prime}-15+6 s}+\frac{-1+I^{\prime} /\left(i_{1}^{\prime} i_{2}^{\prime}\right)}{7 I^{\prime}-24+6 s} \\
& \quad+\frac{1}{I^{\prime}-3+s}\left(-1+\frac{1}{s+1}+\frac{1}{6 I^{\prime}-15+6 s}+\frac{1}{7 I^{\prime}-24+6 s}\right) .
\end{aligned}
$$

In particular, $s_{0}=\left(15-6 I^{\prime}\right) / 6$ is a candidate pole of $Z(s)$, such that $\exp \left(2 \pi i s_{0}\right)=$ $-1 \notin$ Eig.

The point is that, for any $I^{\prime}$, the residue of this candidate pole is zero, that is, this is a fake candidate pole, not a pole. In particular, for $I^{\prime}=7$ we get no contradiction, and for some other special choices of $I^{\prime}$ we get plenty of nonallowed forms for which all the poles provide eigenvalues. For example, for $I^{\prime} \equiv 0(\bmod 6)$ all the poles are integers.

(d) Let us consider the allowed form as in (c) given by $i_{1}=1, i_{2}=3$ and $I^{\prime}=7$. Then $Z$ has a pole $s_{0}$ with $\exp \left(2 \pi i s_{0}\right)=\exp (-2 \pi i / 6)$.

The other root $\exp (2 \pi i / 6)$ of $t^{2}-t+1$ can be realized by the following allowed form. Consider $i_{1}=1, i_{2}=1$ and $I^{\prime}=7$. Then $v_{1}=-1, v_{0}=0, v_{1}^{\prime}=1$ and

$$
Z(s)=\frac{4}{6 s-1}-\frac{1}{s+1}+\frac{1}{6 s+1}\left(-1+\frac{7}{i_{1}^{\prime} i_{2}^{\prime}}\right)+\frac{12}{(6 s-1)(6 s+1)} .
$$

One immediately verifies that all candidate poles are indeed poles. Hence, in fact, the poles of this unique zeta function hit all eigenvalues of Eig. 


\section{Plane curves}

In this section we treat the case when $(X, 0)$ is smooth, that is, $f$ is a plane curve singularity. We will fix some local coordinates $(x, y)$ of $(X, 0)$.

Although the results of Section 7 generalize some of the statements of the present section, we prefer to provide some details in this particular case too, since some of the (much shorter) arguments might be of interest for specialists of plane curve germs. Moreover, we also show how the classical situation $\left(W^{\prime}=0\right)$ is included in our general treatment.

There is another reason to separate the plane curve case. The proof of the abundance of the allowed forms for general $(X, 0)$ (which allows to realize all the monodromy eigenvalues) will be proved under a technical assumption regarding $\Gamma(F)$ (namely, the semigroup condition). Although this condition is satisfied by splice diagrams of plane curve singularities (cf Remark 2.2.6(1)), it is natural to see how the case of plane curves runs independently of this condition, just using their standard properties.

\subsection{The standard form is allowed}

In this section we verify that the standard form is allowed, and hence we reprove the "classical monodromy conjecture" for plane curve singularities.

6.1.1 Proposition The standard differential form $d x \wedge d y$ (corresponding to the divisor $W^{\prime}=0$ on $\left.X\right)$ is allowed for any (plane) curve singularity $f$ on $(X, 0)=$ $\left(\mathbb{C}^{2}, 0\right)$.

Proof We use the minimal embedded resolution $\pi: \tilde{X} \rightarrow X$ of $f$ and show that the diagram $\Gamma:=\Gamma_{\pi}(X, F=\operatorname{div}(f), W=0)$ is allowed. Note that thus the decorations $i_{a}-1=0$ for all $a \in \mathcal{A}_{W}$.

Recall that on any star-shaped subdiagram of $\Gamma$ without boundary vertex the allowedness condition is trivially satisfied. If a star-shaped subdiagram contains exactly one boundary vertex, which by assumption is a boundary vertex of $\Gamma$ too (before the splice operation), then the corresponding leg decoration (being $>1$ ) does not divide the associated $i_{a}(=1)$, hence the allowedness condition is satisfied again.

It is well known that other star-shaped subdiagrams can arise from at most one connected part of $\Gamma$ that has the following form:

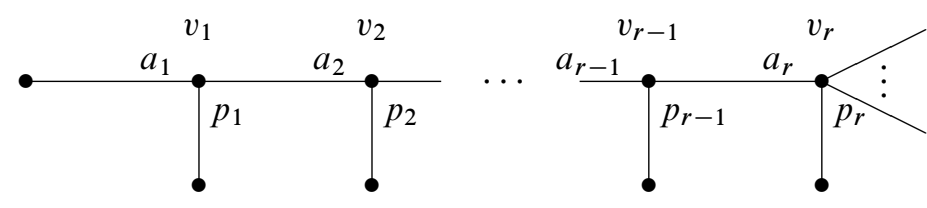


This has the following type of splice subdiagrams (where $2 \leq k \leq r-1$ ):
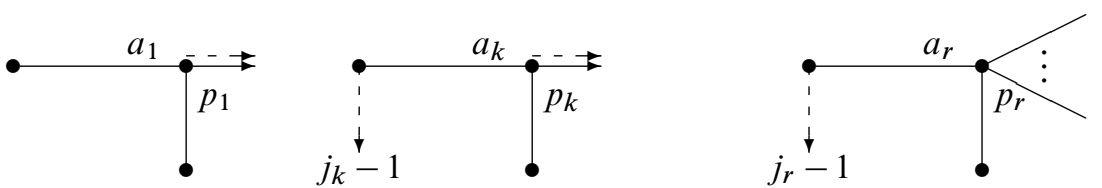

Here the leg with decoration $p_{r}$ is optional. If it does not occur, we put formally $p_{r}=1$. When $r=1$ it must occur, otherwise we are in the situation discussed just before. We will use the positivity of the edge determinants, saying in this case that $a_{k}>a_{k-1} p_{k-1} p_{k}$ for $k=2, \ldots, r$.

For the first diagram the allowedness is automatically satisfied.

In the spliced star-shaped subdiagrams around the vertices $v_{k}, 2 \leq k \leq r$, it is a priori possible that $a_{k} \mid j_{k}$ for some $k$. If furthermore $j_{k} \neq a_{k}$, the allowedness condition would be violated. We will show however that $-a_{k}<j_{k}<0$ for $k=2, \ldots, r$, and this will finish the proof.

More precisely, we verify by induction that $j_{k}<0$ and $\left|j_{k}\right|<a_{k} / p_{k}$ for $k=2, \ldots, r$. First by (3.1.8) we have that $j_{2}=p_{1}+a_{1}-a_{1} p_{1}$. Hence $j_{2}<0$ and $\left|j_{2}\right|<a_{1} p_{1}<$ $a_{2} / p_{2}$.

Take now $k \in\{2, \ldots, r-1\}$. In this case (3.1.11) yields

$$
j_{k+1}=p_{k} j_{k}+a_{k}-a_{k} p_{k}
$$

The induction hypothesis says that $j_{k}<0$ and $\left|p_{k} j_{k}\right|<a_{k}$. Consequently also $j_{k+1}<0$ and $\left|j_{k+1}\right|<a_{k} p_{k}<a_{k+1} / p_{k+1}$.

This together with Theorem 5.1.6 give an alternative proof of the classical monodromy conjecture for curves (see Loeser [14] for the original proof of a stronger result in the context of $p$-adic zeta functions, and Rodrigues [24] for a direct proof).

6.1.2 Corollary For any plane curve singularity $f$, if $s_{0}$ is a pole of the topological zeta function $Z(f ; s)$, then $\exp \left(2 \pi i s_{0}\right)$ is a monodromy eigenvalue of $f$ at some point of $\{f=0\}$.

\subsection{A technical lemma}

In the remaining part of this section we show that any monodromy eigenvalue of a given $f$ can be generated by poles of different $Z(f, W)$ with $W$ allowed. The proof is given in several steps. This section contains two technical partial steps, Lemma 6.2.1 and 
Proposition 6.2.5, targeting those subdiagrams from where the extension of the allowed forms is harder (compare with Remark 4.2.4(b)). The main result is Theorem 6.3.1.

We formulate and prove the following lemma in the context of an arbitrary diagram $\Gamma(F)$ (corresponding to an effective divisor on an IHS germ $(X, 0)$ ). The proof in the plane curve case is not easier, and we will need the general statement in the next section as well.

6.2.1 Lemma Suppose that $\Gamma=\Gamma(F)$ is star-shaped. Let the central node be connected to $n$ boundary vertices and $r$ arrowheads whose supporting edges have decorations $\left\{d_{\ell}\right\}_{\ell=1}^{n}$ and $\left\{p_{\ell}\right\}_{\ell=1}^{r}$, respectively. Here $r \geq 1, n \geq 0$, and $r+n \geq 3$.

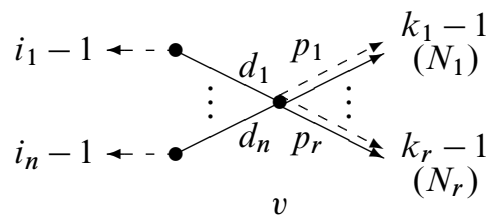

Let $\lambda$ be a root of the Alexander polynomial $\Lambda_{\Gamma}(t)$ of $\Gamma$. Then there exist infinitely many (even infinitely many effective) allowed $P$-divisors $W$ for $\Gamma$ (corresponding to the decorated dashed arrows on the diagram) admitting a pole $s_{0}$ of the topological zeta function $Z(\Gamma(F, W) ; s)$, such that $\exp \left(2 \pi i s_{0}\right)=\lambda$.

Proof Denote $D:=\prod_{\ell=1}^{n} d_{\ell}$ and $P:=\prod_{\ell=1}^{r} p_{\ell}$. For any $P$-divisor $W$, that is, for any set of decorations $k_{1}, \ldots, k_{r}, i_{1}, \ldots, i_{n}$ (with the $i_{\ell} \neq 0$ ), we have that $Z(s):=Z(\Gamma(F, W) ; s)$ has the form

$$
\frac{1}{v+s N}\left(2-r-n+\sum_{\ell=1}^{n} \frac{d_{\ell}}{i_{\ell}}+\sum_{\ell=1}^{r} \frac{p_{\ell}}{k_{\ell}+s N_{\ell}}\right)
$$

where

(6.2.2) $N=D \sum_{\ell=1}^{r} \frac{P}{p_{\ell}} N_{\ell} \quad$ and $\quad v=D \sum_{\ell=1}^{r} \frac{P}{p_{\ell}} k_{\ell}+P \sum_{\ell=1}^{n} \frac{D}{d_{\ell}} i_{\ell}-(r+n-2) D P$.

If the candidate pole $-v / N$ is equal to some $k_{\ell} / N_{\ell}$, then it is a pole of order two. Otherwise, we consider its residue, which is (up to a factor $N$ ) equal to

$$
\mathcal{R}:=2-r-n+\sum_{\ell=1}^{n} \frac{d_{\ell}}{i_{\ell}}+\sum_{\ell=1}^{r} \frac{p_{\ell}}{k_{\ell}-(v / N) N_{\ell}} .
$$


One easily verifies that this expression is not identically zero as function in the $r+n(\geq 3)$ variables $i_{\ell}$ and $k_{\ell}$. Hence $-v / N$ is a pole of $Z(s)$ as soon as the algebraic equation $\mathcal{R}=0$ is not satisfied.

We consider three cases (depending on the value of $r$ ) for the roots of $\Lambda_{\Gamma}(t)$, which is given by

$$
\frac{\left(t^{N}-1\right)^{r+n-2}}{\prod_{\ell=1}^{n}\left(t^{N / d_{\ell}}-1\right)},
$$

except when $r=1$, where we must multiply this expression by $t^{N_{1}}-1$.

- If $r \geq 3$, then its roots are all the $N$-th roots of unity.

- If $r=2$, then its roots are all $N$-th roots of unity that are not $\left(N / d_{\ell}\right)$-th roots of unity simultaneously for all $\ell=1, \ldots, n$. In other words all $\exp \left(2 \pi i \frac{u}{N}\right)$ for which $u \not \equiv 0 \bmod d_{\ell}$ for at least one $d_{\ell}$.

- If $r=1$, then its roots are all $N$-th roots of unity that are not $\left(N / d_{\ell}\right)$-th roots of unity simultaneously for (at least) $n-1$ indexes $\ell=1, \ldots, n$. In other words all $\exp \left(2 \pi i \frac{u}{N}\right)$ for which $u \not \equiv 0 \bmod d_{\ell}$ for at least two different $d_{\ell}$.

Fix a root $\lambda=\exp \left(2 \pi i \frac{u}{N}\right)$ of $\Lambda_{\Gamma}(t)$. Since the numbers $p_{1}, \ldots, p_{r}, d_{1}, \ldots, d_{n}$ are pairwise coprime, there exist integers $k_{1}, \ldots, k_{r}, i_{1}, \ldots, i_{n}$ (all positive if we desire so) such that $v$ in (6.2.2) satisfies $v \equiv u \bmod N$. When $r=2$ or $r=1$, the restrictions on the given $u$ imply that $d_{\ell} \nmid i_{\ell}$ for at least one or at least two indexes $\ell$, respectively. Hence in each case the constructed $W$ is allowed for $\Gamma$.

Since we can choose the numbers $k_{1}, \ldots, k_{r}, i_{1}, \ldots, i_{n}$ freelymod $N$, it is clear that we can find infinitely many such sets (in $\mathbb{Z}$ or in $\mathbb{Z}_{>0}$ ) that satisfy $\mathcal{R} \neq 0$, and hence $s_{0}=-v / N$ is then a pole of $Z(s)$ satisfying $\exp \left(2 \pi i s_{0}\right)=\lambda$.

6.2.3 Remark Assume that above $r=1$ with $p_{1}=1$ and $n=2$. Set $s_{0}=-v / N$. The fact that $\lambda=\exp \left(2 \pi i s_{0}\right)$ is a root of $\Lambda_{\Gamma}(t)$ is equivalent to $d_{1} \nmid i_{1}$ and $d_{2} \nmid i_{2}$. On the other hand, $\mathcal{R}=0$ if and only if $\left(d_{1}-i_{1}\right)\left(d_{2}-i_{2}\right)=0$. Hence, if $\lambda$ is a root, then $s_{0}$ is a pole of $Z(s)$ (for any allowed $W$ ); see also [19, (3.4)].

6.2.4 Now we return to plane curve singularities and we target that subdiagram of the minimal splice diagram whose star-shaped components after splicing have only one ordinary arrowhead; see also Remark 4.2.4(b).

6.2.5 Proposition Consider the subdiagram (given below) of the minimal embedded resolution diagram $\Gamma=\Gamma_{\pi}(X, F)$ of a plane curve germ, determining a $P$-divisor $F$. Here $r \geq 2$ and the leg with decoration $p_{r}$ is optional. 
Fix $k \in\{1, \ldots, r-1\}$, consider the star-shaped subdiagram $S_{k}=\Gamma\left(F_{k}\right)$ around $v_{k}$ and fix a root $\lambda$ of the Alexander polynomial $\Lambda_{S_{k}}(t)$. Then there exist infinitely many allowed divisors $W_{k}$ for $S_{k}$, such that if $\left(N_{k}, v_{k}-1\right)$ denote the decorations of $v_{k}$ as above associated with $S_{k}$ and $W_{k}$, then

(0) $s_{0}=-v_{k} / N_{k}$ is a pole of $Z\left(F_{k}, W_{k} ; s\right)$,

(1) $\exp \left(2 \pi i s_{0}\right)=\lambda$,

(2) $W_{k}$ can be extended to an allowed divisor on the whole diagram $\Gamma$.

The subdiagram of $\Gamma$ is

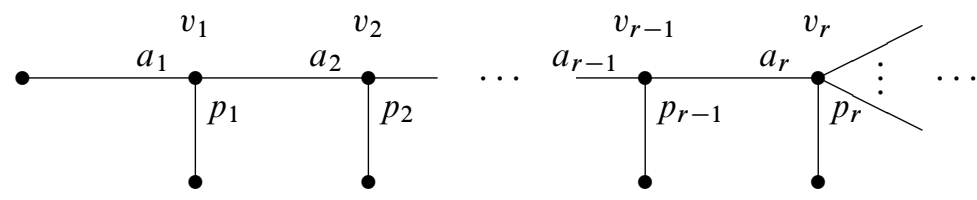

with spliced star-shaped subdiagrams (where we insert the wanted $W_{k}$ 's too):

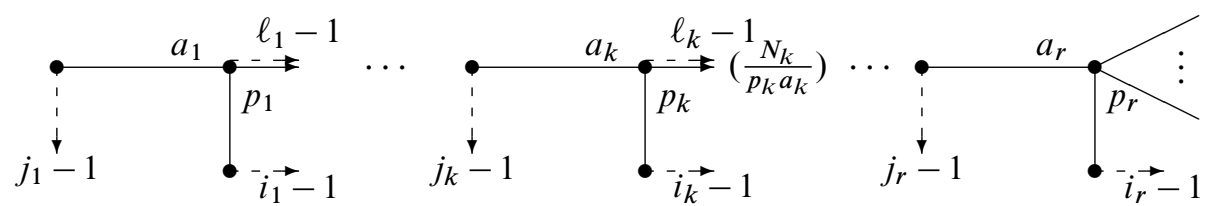

Proof From Lemma 6.2.1 we know that infinitely many $W_{k}$ satisfying (0) and (1) exist, but in order to satisfy also (2), we will specify choices. Write $\lambda$ as $\lambda=$ $\exp \left(2 \pi i\left(u / N_{k}\right)\right)$, where (since it is a root of $\left.\Lambda_{S_{k}}(t)\right) a_{k} \nmid u$ and $p_{k} \nmid u$. We choose the decorations $i_{k}, j_{k}$ and $\ell_{k}$ such that

$$
v_{k}=i_{k} a_{k}+j_{k} p_{k}+\left(\ell_{k}-1\right) a_{k} p_{k} \equiv u\left(\bmod N_{k}\right),
$$

and, moreover

$$
0<i_{k} a_{k}+j_{k} p_{k}-a_{k} p_{k}<a_{k} p_{k} .
$$

This is possible since $a_{k} p_{k} \mid N_{k}$, and since we can choose $\ell_{k}$ freely $\bmod N_{k} /\left(a_{k} p_{k}\right)$. By Remark 6.2.3 we know that $-v_{k} / N_{k}$ is a pole of $Z\left(F_{k}, W_{k} ; s\right)$.

We claim that we can choose inductively $\left\{i_{m}, \ell_{m}\right\}$ for $m=k+1, \ldots, r-1$ such that $\left|j_{m+1}\right|<a_{m} p_{m}$ for $m=k, \ldots, r-1$. Then for these $m$ this yields $\left|j_{m+1}\right|<a_{m} p_{m}<$ $a_{m+1}$ and thus $a_{m+1} \nmid j_{m+1}$. By Remark 4.2.4 this ensures that we can extend $W_{k}$ further to obtain an allowed divisor $W$ on the whole diagram $\Gamma$. 
We now prove the claim. By (3.1.11) we have

$$
\ell_{m}=i_{m+1}+p_{m+1}\left(\ell_{m+1}-1\right)
$$

for $m=k, \ldots, r-2$, and

$$
j_{m+1}=i_{m} a_{m}+j_{m} p_{m}-a_{m} p_{m}
$$

for $m=k, \ldots, r-1$. In particular, we know already from (6.2.6) that $\left|j_{k+1}\right|<a_{k} p_{k}$. When some $\left\{i_{m}, \ell_{m}\right\}$ is constructed we take each time $i_{m+1}$ and $\ell_{m+1}$ in (6.2.7) such that $1 \leq i_{m+1} \leq p_{m+1}$. Then it follows from (6.2.8) and the inductive argument (and the positivity of the edge determinant) that indeed $\left|j_{m+1}\right|<a_{m} p_{m}$ for all $m=$ $k+1, \ldots, r-1$.

\subsection{Abundance of the allowed forms}

Now we are ready to prove the theorem regarding the abundance of the allowed forms.

6.3.1 Theorem Let $(X, 0)$ be a smooth surface germ and $f$ an analytic function on $X$, determining a (plane) curve singularity. Let $\lambda$ be a monodromy eigenvalue of $f$ at a point of $\{f=0\}$. Then there exist infinitely many allowed $P$-divisors $W$ for $(X, \operatorname{div}(f))$, and for each of them a pole $s_{0}$ of the topological zeta function $Z(f, W ; s)$ such that $\exp \left(2 \pi i s_{0}\right)=\lambda$.

Proof Let $\Gamma(F)$ be the diagram of the minimal embedded resolution of $f$.

(1) Suppose first that $\lambda$ is a monodromy eigenvalue at a point $b \in\{f=0\}$, with $b \neq 0$. Writing $\operatorname{div}(f)=\sum_{a \in \mathcal{A}_{F}} N_{a} F_{a}$, this means that $\lambda$ is a $N_{a}$-th root of unity for some $N_{a}$. Fix such an $a \in \mathcal{A}_{F}$; so $\lambda=\exp \left(2 \pi i\left(-u / N_{a}\right)\right.$ ) for some (fixed) $u \in\left\{1, \ldots, N_{a}\right\}$.

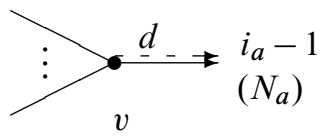

Consider the star-shaped subdiagram $S_{v}=\Gamma\left(F_{v}\right)$ around the vertex $v$, to which the arrowhead $a$ is attached. Choose an allowed $P$-divisor $W_{v}$ for $S_{v}$ with decoration $i_{a} \equiv$ $u \bmod N_{a}$, such that moreover $i_{a} / N_{a} \neq i_{a^{\prime}} / N_{a^{\prime}}$ for all (eventual) other arrowheads $a^{\prime}$ on $S_{v}$. (Here we have infinitely many such choices.) Then either $-i_{a} / N_{a}$ is a pole of order two of $Z\left(F_{v}, W_{v} ; s\right)$, or the contribution of $a$ to this zeta function is

$$
\frac{d}{\left(v_{v}+s N_{v}\right)\left(i_{a}+s N_{a}\right)}
$$

and hence the residue of $-i_{a} / N_{a}$ is nonzero. 
We surely can extend $W_{v}$ to an allowed divisor $W$ on the whole of $\Gamma(F)$ (see Remark 4.2.4), and doing so we do not use the value $i_{a}$. (For this see the proof of Proposition 4.2.2; with the notation of that proof, from $\Gamma_{R}$ only $i^{\prime}$ was used.) If $-i_{a} / N_{a}$ is not a pole of order two and $i_{a} / N_{a}$ would be equal to some value $v_{w} / N_{w}, w \in\left(\mathcal{N} \cup \mathcal{A}_{F}\right) \backslash\{a\}$, then we can add to $i_{a}$ some multiple of $N_{a}$ in order to avoid this. This way we are sure that $-i_{a} / N_{a}$ is a pole of $Z(f, W ; s)$.

(2) Suppose now that $\lambda$ is not as in (1); hence it is a root of the Alexander polynomial $\Lambda_{\Gamma(F)}(t)$. By Proposition 3.3.3 there is at least one node $w \in \mathcal{N}$ such that $\lambda$ is a root of $\Lambda_{S_{w}}(t)$, where $S_{w}=\Gamma\left(F_{w}\right)$ is the star-shaped subdiagram around $w$. By Lemma 6.2.1 and Proposition 6.2.5 there exist infinitely many allowed $P$-divisors $W_{w}$ for $S_{w}$, and for each of them a pole $s_{0}$ of $Z\left(F_{w}, W_{w} ; s\right)$ such that $\exp \left(2 \pi i s_{0}\right)=\lambda$ and $W_{w}$ can be extended to an allowed divisor $W$ on the whole diagram $\Gamma(F)$. Indeed, the possible obstruction to extend $W_{w}$, as described in Remark 4.2.4, is removed in Proposition 6.2.5.

If $s_{0}$ is a pole of order two of $Z\left(F_{w}, W_{w} ; s\right)$, then it is a pole of order two of $Z(s)=$ $Z(f, W ; s)$ too, hence we are done. Otherwise, there is a potential problem when the following situation occurs: $s_{0}$ is not a pole of order two, $s_{0}=-v_{w} / N_{w}$ for a subset $\mathcal{N}^{\prime} \subset \mathcal{N}$ containing at least two nodes $w$ of $\Gamma(F)$, such that for each $w \in \mathcal{N}^{\prime}$ the local residue-contribution (to the total residue of $\left.s_{0}\right) \mathcal{R}_{w} \neq 0$, and $\sum_{w \in \mathcal{N}^{\prime}} \mathcal{R}_{w}=0$. In this case $s_{0}$ is not a pole of $Z(s)$, although it is a pole of several $Z\left(F_{w}, W_{w} ; s\right)$.

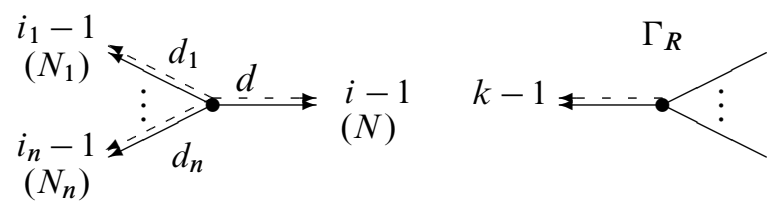

Take an "extreme" node $v \in \mathcal{N}^{\prime}$ (the node of the left diagram above), meaning that it is a boundary vertex of the full subdiagram of $\Gamma$ generated by $\mathcal{N}^{\prime}$. Consider the star-shaped subdiagram $S_{v}$ of $\Gamma=\Gamma(F, W)$ around $v$, where the edge $e$ with decoration $d$ is in the direction of the other nodes in $\mathcal{N}^{\prime}$, and the diagram $\Gamma_{R}$, obtained after splicing $\Gamma$ along $e$. (It is not necessary to have arrowheads at all the legs of $S_{v}$, as it is indicated in the above diagram; in those cases we put formally $N_{\ell}=0$ or $N=0$.)

Denote $D:=\prod_{j=1}^{n} d_{j}$. By (2.3.5) and (3.1.11) (or from (6.3.2) below) we know that $v_{v}$ depends only on $i$ and $k=\sum_{j=1}^{n}\left(D / d_{j}\right) i_{j}-(n-1) D$, and not on the actual (separate) values of $i_{1}, \ldots, i_{n}$. The residue-contribution $\mathcal{R}_{v}$ though depends on these values.

We claim that we can modify $i_{1}, \ldots, i_{n}$ keeping $k$ (and hence $v_{v}$ ) fixed, but changing $\mathcal{R}_{v}$, such that the newly created divisor (determined by these new $i_{1}, \ldots, i_{n}$ ) has the following properties: it agrees with the old $P$-divisor on $\Gamma_{R}$, and can be extended from $S_{v} \cup \Gamma_{R}$ further "to the left" to a new allowed P-divisor $\widetilde{W}$ on the whole diagram. 
In that way the new value becomes $\sum_{v \in \mathcal{N}^{\prime}} \mathcal{R}_{v} \neq 0$. If "on the left" there are no nodes $v^{\prime}$ with "new value" $v_{v^{\prime}} / N_{v^{\prime}}=s_{0}$, we are done since then this sum is the (total) residue of $s_{0}$ for $Z(s)$. We are still done if the sum of $\sum_{v \in \mathcal{N}^{\prime}} \mathcal{R}_{v}$ and all new residue-contributions of these $v^{\prime}$ is nonzero. Otherwise, we repeat the argument, replacing $v$ in the claim by such a new (extreme) node $v^{\prime}$. This process must stop by finiteness of the diagram.

(3) We now prove the claim. We start with two observations.

(i) Since there are at least two nodes in $\mathcal{N}^{\prime}$, we can always assume that there is an ordinary arrowhead at the leg of $S_{v}$ with decoration $d$, that is, that $N \neq 0$. Then, with the terminology of Remark 4.2.4(b), we can extend any $P$-divisor on $\Gamma_{R}$ "unconditionally" to the left. More precisely, the specific situation/problem of Proposition 6.2.5 will never occur; we can always simply follow the procedure in the proof of Proposition 4.2.2.

(ii) We must be sure that we can modify $i_{1}, \ldots, i_{n}$ while extending from $\Gamma_{R}$, considering the extension procedure described in the proof of Proposition 4.2.2. In this procedure there is no room to modify $i_{1}, \ldots, i_{n}$ only if (after renumbering) $i_{\ell}$ must be chosen as $i_{\ell}=d_{\ell}$ for $\ell \leq n-1$. But in this case we would have that $\mathcal{R}_{v}=0$ (see Lemma 5.1.1), contradicting our assumption.

By (2.3.5), (3.1.11) and (2.2.3) we have

$$
\begin{aligned}
v_{v} & =\sum_{j=1}^{n} d\left(D / d_{j}\right) i_{j}+D i-(n-1) d D=d k+D i, \\
N_{v} & =\sum_{j=1}^{n} d\left(D / d_{j}\right) N_{j}+D N .
\end{aligned}
$$

We have further that

$$
N_{v} \mathcal{R}_{v}=1-n+\sum_{j=1}^{n} \frac{d_{j}}{i_{j}-\left(v_{v} / N_{v}\right) N_{j}}+\frac{d}{i-\left(v_{v} / N_{v}\right) N} .
$$

Case $n \geq 3$ We replace the triple $\left(i_{1}, i_{2}, i_{3}\right)$ by $\left(i_{1}+x d_{1}, i_{2}+y d_{2}, i_{3}-(x+y) d_{3}\right)$ where $x, y \in \mathbb{Z}$. Then $v_{v}$ does not change, but the three corresponding terms in $N_{v} \mathcal{R}_{v}$ are replaced by

$$
\frac{d_{1}}{i_{1}+x d_{1}-\left(v_{v} / N_{v}\right) N_{1}}+\frac{d_{2}}{i_{2}+y d_{2}-\left(v_{v} / N_{v}\right) N_{2}}+\frac{d_{3}}{i_{3}-(x+y) d_{3}-\left(v_{v} / N_{v}\right) N_{3}} .
$$

It is easy to see that this expression is not constant as function in $x$ and $y$; hence we can choose appropriate $x$ and $y$ in $\mathbb{Z}$ such that the "new" $\mathcal{R}_{v}$ is different from the original one. (Note that divisibility of $i_{\ell}$ by $d_{\ell}$ does not change, so we don't destroy 
allowedness.) We then extend this new $P$-divisor from $S_{v} \cup \Gamma_{R}$ further to an allowed divisor on the whole diagram.

Case $\boldsymbol{n}=\mathbf{2}$ We replace the pair $\left(i_{1}, i_{2}\right)$ by $\left(i_{1}+x d_{1}, i_{2}-x d_{2}\right)$ where $x \in \mathbb{Z}$. Again $v_{v}$ does not change, and now the two corresponding terms in $N_{v} \mathcal{R}_{v}$ are replaced by

$$
\frac{d_{1}}{i_{1}+x d_{1}-\left(v_{v} / N_{v}\right) N_{1}}+\frac{d_{2}}{i_{2}-x d_{2}-\left(v_{v} / N_{v}\right) N_{2}} \text {. }
$$

When this expression is not constant in $x$, we conclude as above. It is constant in $x$ if and only if it is identically zero if and only if

$$
d_{1} i_{2}+d_{2} i_{1}-\frac{v_{v}}{N_{v}}\left(d_{1} N_{2}+d_{2} N_{1}\right)=0
$$

Suppose this identity holds. Then the formulas for $v_{v}$ and $N_{v}$ above easily yield that $v_{v} / N_{v}=(i-d) / N$. But then the (original) $N_{v} \mathcal{R}_{v}$ would be equal to

$$
-1+\frac{d}{i-\left(v_{v} / N_{v}\right) N}=0,
$$

contradicting the assumption.

6.3.3 Remark For plane curve singularity germs $f$, the associated allowed $W$ in Theorem 6.3.1 are always divisors of differential forms $\omega$. From the proof of the more general Theorem 7.4.23, we will see that there exist moreover infinitely many effective allowed divisors $W$ doing the job in the theorem, corresponding here in the plane curve case to holomorphic differential forms $\omega$.

\section{Diagrams $\Gamma(F)$ with the semigroup condition}

\subsection{The semigroup condition}

Let us fix a diagram $\Gamma(F)$. The reader is invited to recall the definition of the semigroup condition associated with $\Gamma(F)$ from Section 2.2.5.

The semigroup condition of $\Gamma(F)$ is equivalent with the following property: for any edge $e$ (as in the following diagram; see also Section 3.1.5), such that $\mathcal{A}_{F, L}=\varnothing$, $d^{\prime}$ is in the semigroup $\mathcal{S}_{e}$ generated by $\left\{l_{e w}\right\}_{w}$ where the index $w$ runs over all the boundary vertices of $\Gamma_{L}$. (For the definition of $l_{e w}$ see Section 3.1.2.)

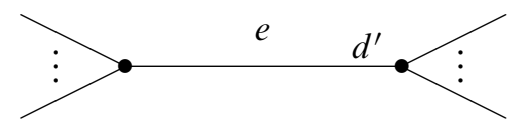


This condition appears naturally in the context of splice quotient singularities, introduced by Neumann and Wahl $[20 ; 21]$. For some special diagrams this condition is automatically satisfied. For example, if $\Gamma$ represents a rational germ (which in the context of IHS germs is equivalent with the fact that $\Gamma$ represents either the smooth or the $E_{8}$ germ), and the diagram is not necessarily minimal and $F$ is arbitrary, then $\Gamma(F)$ has the semigroup condition. Another case is when $\Gamma$ is minimal and it represents a minimally elliptic (automatically Gorenstein) singularity. These facts follow from the "End Curve Theorem" of Neumann and Wahl [22] and Okuma [23].

It is convenient to denote by $\mathcal{S}\left\langle g_{1}, \ldots, g_{t}\right\rangle$ the subsemigroup of $\mathbb{N}$ generated by $g_{1}, \ldots g_{t}$.

\subsection{Preliminary arithmetical properties}

Here we gather some arithmetical properties which will be useful in the proofs of the main results of this section (listed in the next subsection).

7.2.1 Lemma Let $d_{1}, \ldots, d_{n}$ be pairwise coprime positive integers, and denote $D:=\prod_{j=1}^{n} d_{j}$. Then the following two facts hold.

(a) There exist no positive integers $m_{j}$ such that

$$
\sum_{j=1}^{n} m_{j} \frac{D}{d_{j}}=(n-1) D .
$$

(b) If $d \in \mathcal{S}\left\langle D / d_{1}, \ldots, D / d_{n}\right\rangle, d>0$ and $d \mid D$, then $\left(D / d_{j}\right) \mid d$ for some $j \in$ $\{1, \ldots, n\}$.

Proof For (a), in such an equality we would have that $d_{j} \mid m_{j}$ for all $j=1, \ldots, n$. But then the left hand side would be at least $n D$. In (b), by assumption, we can write $d$ in the form

$$
d=\sum_{j=1}^{n} m_{j} \frac{D}{d_{j}},
$$

where all $m_{j}$ are nonnegative integers, and also $d=\prod_{j=1}^{n} \bar{d}_{j}$ with $\bar{d}_{j} \mid d_{j}$ for all $j$. Since the $d_{j}$ are pairwise coprime, (7.2.2) shows that $\bar{d}_{j} \mid m_{j}$ for all $j$. Writing $d$ as $\bar{d}_{j} \prod_{\ell \neq j} \bar{d}_{\ell}$, we conclude that $d$ divides $m_{j} D / d_{j}$ for all $j$. If at least two of the numbers $m_{j}$ would be nonzero, say $m_{1} \neq 0$ and $m_{2} \neq 0$, we obtain the contradiction

$$
d \geq m_{1} \frac{D}{d_{1}}+m_{2} \frac{D}{d_{2}} \geq d+d .
$$

Hence exactly one $m_{j}$ is nonzero, implying then that $\left(D / d_{j}\right) \mid d$. 
Recall that a diagram $\Gamma$ is called minimal if all the decorations $d_{v e}$ are strictly greater than 1 , provided that $e$ connects the node $v$ with a boundary vertex.

7.2.3 Proposition Let $\Gamma(F)$ be a splice diagram as in Section 2.2 (hence with $\left.W^{\prime}=0\right)$ and minimal in the above sense. Let $e$ be an edge connecting two nodes such that $\mathcal{A}_{F, L}=\varnothing$, and set $i^{\prime}-1$ the multiplicity of the induced dashed arrowhead at $v_{R}$ after splicing $\Gamma$ along $e$ as in Section 3.1.5.

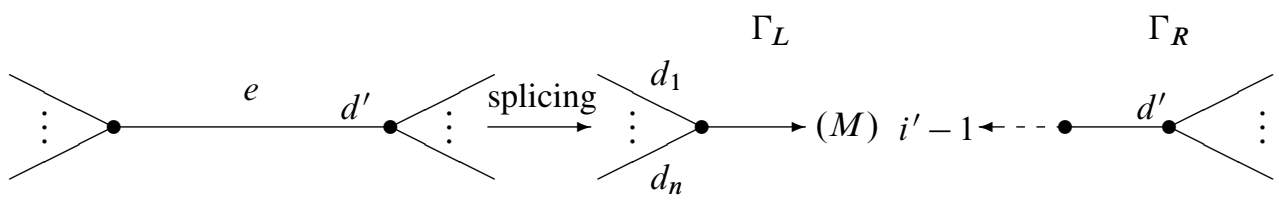

Then

(1) $i^{\prime}<0$.

(2) Assume that the semigroup condition is satisfied in $\Gamma(F)$ (at least for the edge $e$ and the edges $e_{L}$ sitting in $\Gamma_{L}$ ). (This means that $d^{\prime}$ is in the semigroup $\mathcal{S}_{e}$ generated by $\left\{l_{e w}\right\}_{w}$ where the index $w$ runs over all the boundary vertices of $\Gamma_{L}$, and there are similar inclusions for all edges $e_{L}$ of $\Gamma_{L}$.) Then $-i^{\prime} \notin \mathcal{S}_{e}$. Hence, $d^{\prime} \nmid i^{\prime}$.

Proof (1) Set $D:=\prod_{j=1}^{n} d_{j}$. We proceed by induction on the number of nodes in $\Gamma_{L}$.

Suppose first that $v_{L}$ is the only node of $\Gamma_{L}$. By Equation (3.1.8) we have that $i^{\prime}=\sum_{j=1}^{n} D / d_{j}-(n-1) D$. When $n=2$, this is $d_{1}+d_{2}-d_{1} d_{2}$ and thus negative. When $n>2$, then by minimality $d_{j} \geq 2$, hence $\sum 1 / d_{j} \leq n / 2<n-1$, therefore $i^{\prime}<0$ again.

We suppose now that $\Gamma_{L}$ contains at least two nodes. From (3.1.8) we can write $i^{\prime}$ as

$$
i^{\prime}=\sum_{j=1}^{n} \frac{D}{d_{j}}\left(\sum_{w \in \mathcal{V}_{j}}\left(2-\delta_{w}\right) \ell_{e_{j} w}\right)-(n-1) D,
$$

where for $j=1, \ldots, n$ the set $\mathcal{V}_{j}$ consists of the vertices of $\Gamma_{L}$ connected (geodesically) to $v_{L}$ through the edge $e_{j}$ with weight $d_{j}$, and $\ell_{e_{j}} w$ is the product of all the decorations adjacent to, but not on, the path from $w$ to $e_{j}$. For all $j$ this sum is either equal to 1 (when $e_{j}$ ends at a boundary vertex), or negative by induction. Since at least one sum is negative, we conclude that $i^{\prime}<0$. 
(2) Denote by $\mathcal{B}_{L}$ and $\mathcal{N}_{L}$ the boundary vertices and nodes, respectively, in $\Gamma_{L}$. We will show the following claim. Let

$$
I:=\sum_{w \in \mathcal{B}_{L}} m_{w} \ell_{e w}+\sum_{w \in \mathcal{N}_{L}}\left(2-\delta_{w}\right) \ell_{\text {ew }},
$$

where all $m_{w} \in \mathbb{Z}_{>0}$. Then $I \neq 0$ and, if $I<0$, then $-I \notin \mathcal{S}_{e}$.

Since $i^{\prime}=I$ when all $m_{w}=1$, and $i^{\prime}<0$ by part (1), the statement then follows.

We now prove the claim, again by induction on the cardinality of $\mathcal{N}_{L}$. If $\mathcal{N}_{L}=\left\{v_{L}\right\}$ then

$$
I=\sum_{j=1}^{n} m_{j} \frac{D}{d_{j}}-(n-1) D,
$$

and this is nonzero by Lemma 7.2.1(a). If $I<0$ and $-I \in \mathcal{S}_{e}$, then $\sum_{j=1}^{n} k_{j}\left(D / d_{j}\right)=$ $(n-1) D$ for some positive integers $k_{j}$, contradicting again Lemma 7.2.1(a).

Let now $\mathcal{N}_{L}$ have at least two elements. Suppose again that $I<0$ and $-I \in \mathcal{S}_{e}$. Then analogously we get that

$$
\sum_{w \in \mathcal{B}_{L}} k_{w} \ell_{e w}=\sum_{w \in \mathcal{N}_{L}}\left(\delta_{w}-2\right) \ell_{e w}
$$

for some positive integers $k_{w}$. We separate $v_{L}$ (with $\delta_{v_{L}}=n+1$ and $\ell_{e v_{L}}=D$ ) on the right hand side, and rewrite this equality as

$$
\sum_{j=1}^{n} \frac{D}{d_{j}}\left[\sum_{w \in \mathcal{B}_{L}^{(j)}} k_{w} \ell_{e_{j} w}+\sum_{w \in \mathcal{N}_{L}^{(j)}}\left(2-\delta_{w}\right) \ell_{e_{j} w}\right]=(n-1) D,
$$

where $\mathcal{B}_{L}^{(j)} \cup \mathcal{N}_{L}^{(j)}=\mathcal{V}_{j}$. By induction the square bracket is nonzero. Moreover, (7.2.4) shows that $d_{j}$ divides the $j$-th square bracket for all $j$. Applying the induction hypothesis on all these terms (and the assumption $d_{j} \in \mathcal{S}_{e_{j}}$ ) yields that all these square brackets are positive. But this contradicts Lemma 7.2.1(a).

We still have to show that $I \neq 0$. Assuming that $I=0$ yields the same expression as in (7.2.4), with the $k_{w}$ replaced by the original $m_{w}$. And then we obtain a contradiction by the same argument.

7.2.5 Remark Let us give the "Milnor number interpretation" of the statement Proposition 7.2.3(1). Consider the splice diagram $\Gamma_{L}$, but replace the multiplicity $M$ of the unique arrowhead by 1 . This represents a fibered knot; let $S$ be its fiber. It is a connected punctured Riemann surface. Let its first Betti number be $\mu$ (the Milnor 
number). Clearly, $\mu$ is even. Since $\Gamma_{L}$ is minimal and nonempty, $\mu \neq 0$ (its proof is basically our proof of (1)). On the other hand, by [2], $i^{\prime}=\chi(S)=1-\mu$, hence $i^{\prime}<0$. The second part also has some "classical" interpretation. Start again with the fact $-i^{\prime}=\mu-1$, and assume that the above diagram represents a plane curve singularity. Then $\mathcal{S}_{e}$ is exactly the semigroup $\mathcal{S}$ of the plane curve, and it is a classical fact that $\mu-1$ is the largest integer not in $\mathcal{S}$.

The point is that in any generalization of $\mu-1 \notin \mathcal{S}$ for more general $\Gamma_{L}$ (as our (2) does) one needs some restriction about $\Gamma_{L}$ : for example, if we have two nodes, the second one in $\mathcal{V}_{1}$, and $d_{1}=1$, then $\mathcal{S}_{e}=\mathbb{N}$.

\section{3 $W=0$ is allowed}

7.3.1 Theorem Let $F$ be a (nonzero) effective divisor on an IHS germ $(X, 0)$, such that the minimal embedded resolution diagram $\Gamma_{\pi}(X, F)$ satisfies the semigroup condition. Then the divisor $W=0$ is allowed for the pair $(X, F)$.

Proof Denoting by $\pi$ this minimal embedded resolution, we will show that the diagram $\Gamma=\Gamma_{\pi}(X, F, W=0)$ is allowed. Note that thus the decorations $i_{a}-1=0$ for all $a \in \mathcal{A}_{W}$.

Recall again that on any star-shaped subdiagram of $\Gamma$ without boundary vertex the allowedness condition is trivially satisfied. If a star-shaped subdiagram contains a boundary vertex, that is an original boundary vertex of $\Gamma$, then the corresponding leg decoration (being $>1$ by minimality) does not divide the associated $i_{a}(=1)$.

If a star-shaped subdiagram contains a boundary vertex, that is created after splicing, that diagram looks like the right diagram in the statement of Proposition 7.2.3, where $i^{\prime}-1$ is the decoration of the constructed dashed arrow attached to that boundary vertex, and $d^{\prime}$ is the corresponding edge weight. Since we showed in Proposition 7.2.3 that $d^{\prime} \nmid i^{\prime}$, the allowedness condition is verified in this case too (hence everywhere) by Addendum 4.2.5.

7.3.2 Example Recall that in Example 4.1.7 we presented a minimal diagram $\Gamma(F)$ for which $W=0$ is not allowed. Hence some kind of restriction is indeed necessary in order to guarantee the allowedness of $W=0$.

7.3.3 Corollary Let $(X, 0)$ be a Gorenstein IHS germ, with nowhere vanishing 2form $\omega_{0}$ on $X \backslash\{0\}$. Let $f$ be a function germ on $(X, 0)$ such that the minimal embedded resolution diagram $\Gamma_{\pi}(X, f)$ satisfies the semigroup condition.

If $s_{0}$ is a pole of the topological zeta function $Z(f ; s)=Z(\operatorname{div}(f), W=0 ; s)$, then $\exp \left(2 \pi i s_{0}\right)$ is a monodromy eigenvalue of $f$ at some point of $\{f=0\}$. 
Proof Immediate from Theorem 7.3.1 and Theorem 5.1.6.

7.3.4 Remark In [24, Theorem 2.2], Rodrigues showed without requiring the semigroup condition that even in the singular setting $\exp \left(2 \pi i s_{0}\right)$ is a monodromy eigenvalue of $f$ provided that the pole $s_{0}$ satisfies $s_{0} \leq 0$. We wish to emphasize that this is a rather strong assumption in the context of singular ambient spaces. Indeed, if we consider a minimal resolution of a noncanonical surface singularity, then the canonical cycle $K$ is nef, and all its coefficients $v-1$ are (strictly) negative, hence the corresponding values $-v / N$ are all nonnegative. We can create negative poles when we have to blow up the minimal resolution in order to get a good resolution of $(X, F)$, so by subgraphs which behave like graphs of plane curve singularities. Usually there are only a few poles like this (although, for plane curves all of them are negative by the very same argument).

\subsection{All eigenvalues are realized by poles}

The main result of the section is based on the following technical proposition.

7.4.1 Proposition Let $(X, 0)$ be an IHS germ and $f$ an analytic function on $X$, such that the minimal embedded resolution diagram $\Gamma=\Gamma_{\pi}(X, f)$ satisfies the semigroup condition. With the notation of Remark 4.2.4(b), let us fix a node $v_{0}$ in $\Gamma_{\mathcal{A}}$, and another node $v_{m}$ not in $\Gamma_{\mathcal{A}}$ at "distance" $m \geq 1$ from $v_{0}$. Consider the diagram $\Gamma_{0}$ given below obtained from $\Gamma$ by cutting via splice-decomposition all the nodes not sitting on the geodesic path connecting $v_{0}$ with $v_{m}$. Here the legs with decorations $d_{0,2}, \ldots, d_{0, n_{0}}$ are optional, and all boundary vertices are either original boundary vertices of $\Gamma$, or are obtained after splicing.
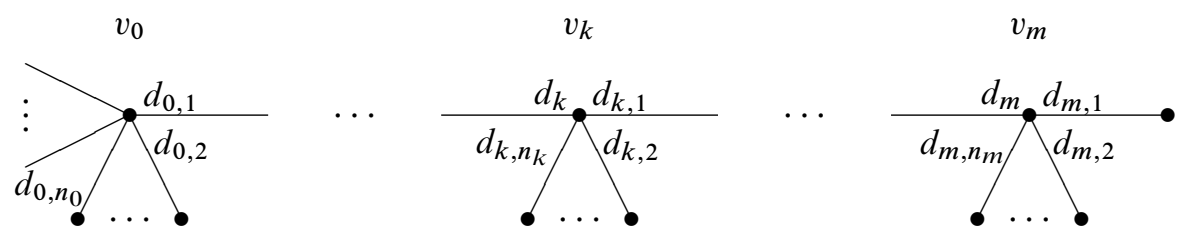

Splice this diagram at the edge $\left(v_{k-1} v_{k}\right), 0<k \leq m$, and denote that splice component which contains $v_{k}, \ldots, v_{m}$ by $\Gamma_{k}=\Gamma_{k}\left(F_{k}\right)$. Let the decoration of the dashed arrowhead not in $\Gamma_{1}$, associated with the splicing along $\left(v_{0} v_{1}\right)$, be $i_{0,1}-1$ (see the picture below).

Fix a root $\lambda$ of the Alexander polynomial $\Lambda_{\Gamma_{m}}(t)$ associated with the star-shaped diagram $\Gamma_{m}$. Then there exist infinitely many allowed divisors $W_{m}$ for $\Gamma_{m}$, such that if $\left(N_{m}, v_{m}-1\right)$ denote the decorations of $v_{m}$ as above associated with $\Gamma_{m}$ and $W_{m}$, then 
(1) $s_{0}=-v_{m} / N_{m}$ is a pole of $Z\left(F_{m}, W_{m} ; s\right)$, with $\exp \left(2 \pi i s_{0}\right)=\lambda$,

(2) $W_{m}$ extends to an allowed divisor on $\Gamma_{1}$, such that $d_{0,1} \nmid i_{0,1}$.

Moreover, infinitely many of these allowed (extended) $W_{m}$ on $\Gamma_{1}$, as well as their further extensions (in the sense of Remark 4.2.4(b)) on the whole of $\Gamma$, may be chosen to be effective.

Note that the above additional nondivisibility property (2) is the key assumption in Addendum 4.2.5.

Proof We proceed in several steps. During the proof $\lambda$ is fixed.

7.4.2 We fix notation for the wanted $W_{m}$ and its extensions on the spliced star-shaped subdiagrams of $\Gamma_{1}$. Moreover, we also consider the decoration $i_{0,1}$ which is part of a potential extension to $\Gamma_{0}$, but it is completely determined by the extension on $\Gamma_{1}$.
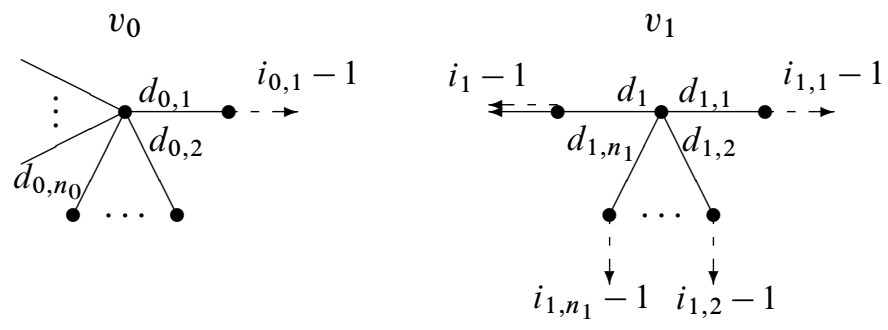

We also set $D_{k}:=\prod_{\ell=1}^{n_{k}} d_{k, \ell}$ and $D_{k}^{*}:=\prod_{\ell=2}^{n_{k}} d_{k, \ell}(1 \leq k \leq m)$. Note that $n_{k} \geq 2$. The semigroup condition for $\Gamma$ implies that for any $k>0$ one has

(7.4.3) $\quad d_{k-1,1}$

$$
\left.\in \mathcal{S}\left\langle\frac{D_{k}}{d_{k, 2}}, \ldots, \frac{D_{k}}{d_{k, n_{k}}}, \frac{D_{k}^{*} D_{k+1}}{d_{k+1,2}}, \ldots, \frac{D_{k}^{*} D_{k+1}}{d_{k+1, n_{k+1}}}, \frac{D_{k}^{*} D_{k+1}^{*} D_{k+2}}{d_{k+2,2}}, \ldots\right\rangle\right) .
$$

The wanted divisor will be constructed by induction. From Lemma 6.2.1 we know that infinitely many $W_{m}$, even infinitely many effective $W_{m}$, satisfying (1) exist (see also Section 7.4.7). Here $W_{m}$ identifies $v_{m}$ by

$$
v_{m}=i_{m-1,1} d_{m}+i_{m} D_{m}
$$

Then, we analyze how an allowed divisor $W_{k+1}$ from $\Gamma_{k+1}$ can be extended over $\Gamma_{k}$. Along this procedure we will use the following identities "around $v_{k}$ " satisfied by any 
extension:

$$
\begin{aligned}
i_{k+1} & =-\left(n_{k}-1\right) d_{k} D_{k}^{*}+i_{k} D_{k}^{*}+\sum_{\ell \geq 2} i_{k, \ell} d_{k} D_{k}^{*} / d_{k, \ell}, \\
i_{k-1,1} & =-\left(n_{k}-1\right) D_{k}+\sum_{\ell \geq 1} i_{k, \ell} D_{k} / d_{k, \ell} .
\end{aligned}
$$

In this procedure we need a deeper understanding of the extensions (compared with Section 4.2), and we need to consider divisors with some special properties, we will call them "strict". The decorations of their nodes satisfy some additional conditions, as it is explained next.

Assume that $W_{k}$ is an allowed divisor on $\Gamma_{k}$ for some $k \geq 1$. The decorations of $W_{k}$ will distinguish the nodes as follows. For some $k^{\prime} \in\{k, \ldots, m\}$, the node $v_{k^{\prime}}$ is called flexible if there are at least two indexes $\ell \in\left\{1, \ldots, n_{k^{\prime}}\right\}$ for which $d_{k^{\prime}, \ell} \nmid i_{k^{\prime}, \ell}$. For $k^{\prime} \in\{k, \ldots, m-1\}$, if $v_{k^{\prime}}$ is not flexible, but $d_{k^{\prime}, \ell}=i_{k^{\prime}, \ell}$ for all $\ell \in\left\{2, \ldots, n_{k^{\prime}}\right\}$ then it is called rigid. Note that not all nonflexible nodes are rigid (see the cases discussed in Remark 4.2.4).

In this proof the nodes of all allowed divisors will be either flexible or rigid.

7.4.7 First we construct an allowed divisor $W_{m}$ which satisfies (1) and is flexible at $v_{m}$. We search for $i_{m}, i_{m, 1}, \ldots, i_{m, n_{m}}$ such that they satisfy the allowedness at $v_{m}$, (7.4.4), (7.4.6) for $k=m$, and $\exp \left(-2 \pi i v_{m} / N_{m}\right)$ is root of the Alexander polynomial $\Lambda_{\Gamma_{m}}(t)$. Since $D_{m} \mid N_{m}$, the last condition implies that

$$
d_{m, \ell} \nmid v_{m} \text { for at least two indexes } \ell \in\left\{1, \ldots, n_{m}\right\} .
$$

We proceed as follows. For any $v_{m}$ with (7.4.8) we find $i_{m-1,1}$ and $i_{m}$ satisfying (7.4.4). This is possible since $\operatorname{gcd}\left(d_{m}, D_{m}\right)=1$. Then we find integers $\left\{i_{m, \ell}\right\}_{\ell=1}^{n_{m}}$ satisfying (7.4.6). This, again, is possible since $\operatorname{gcd}_{\ell}\left(D_{m} / d_{m, \ell}\right)=1$. Since $d_{m, \ell} \mid v_{m}$ if and only if $d_{m, \ell} \mid i_{m-1,1}$ if and only if $d_{m, \ell} \mid i_{m, \ell}$, by (7.4.8) we have that $d_{m, \ell}+i_{m, \ell}$ for at least two indexes, hence $W_{m}$ is flexible at $v_{m}$.

7.4.9 Next we analyze the possibilities how one can extend divisors. Consider an allowed divisor $W_{k+1}$ on $\Gamma_{k+1}(1 \leq k<m)$. Note that it also determines $i_{k, 1}$ by (7.4.6). Extending over $v_{k}$ means that we already know everything over $\Gamma_{k+1}$ and $i_{k, 1}$, and we are searching for $i_{k}$ and $\left\{i_{k, \ell}\right\}_{\ell \geq 2}$ which satisfy the allowedness condition at $v_{k}$ and the identity (7.4.5).

The divisor $W_{k+1}$ and the decorations of $\Gamma_{0}(F)$ contain all the divisibility information, like $d_{k, \ell}$ divides $i_{k, \ell}$ or not, for any extension $W_{k}$ on $\Gamma_{k}$. Indeed, $i_{k, 1}$ and $d_{k, 1}$ are determined by $\Gamma_{0}$ and $W_{k+1}$, and the divisibility conditions $d_{k, \ell} \nmid i_{k, \ell}(\ell \geq 2)$ 
are determined by (7.4.5), since $d_{k, \ell} \nmid i_{k, \ell} \Leftrightarrow d_{k, \ell} \nmid i_{k+1}$. Hence, several crucial divisibility properties of an extension $W_{k}$ on $\Gamma_{k}$ are already decided at the level of its restriction $W_{m}$ on $\Gamma_{m}$. This makes the inductive construction of $W_{k}$, starting from $W_{m}$ "global" and difficult.

In order to guarantee the existence of such an extension $W_{k}$, we will use two types of criteria: $W_{k+1}$ satisfies either $d_{k, 1} \nmid i_{k, 1}$ or $D_{k}^{*} \mid i_{k+1}$ (see Addendum 4.2.5).

If $D_{k}^{*} \mid i_{k+1}$ then it has no flexible extension (but, it might happen that it has several allowed extensions); we take always that unique extension for which $v_{k}$ will be rigid: $d_{k, \ell}=i_{k, \ell}$ for $\ell \geq 2$. Moreover, (7.4.5) and (7.4.6) read as

$$
i_{k}=i_{k+1} / D_{k}^{*} \text { and } \quad i_{k-1,1}=i_{k, 1} D_{k}^{*} .
$$

If $d_{k, 1} \nmid i_{k, 1}$, then for any extension $W_{k}$ the node $v_{k}$ is either flexible or rigid (and the type is decided already at the level of $\left.W_{k+1}\right) ; v_{k}$ is rigid if and only if additionally $D_{k}^{*} \mid i_{k+1}$, the case discussed before. If $v_{k}$ is flexible, then the extension is not unique, it can be modified if it is necessary (and we will do this intensively).

Next, we have to check if the extension has one of the two criteria which guarantee the further extension. We show that if we "modify $W_{k}$ at the closest flexible node", it will satisfy the inductive criteria $d_{k-1,1} \nmid i_{k-1,1}$, provided that the tower of extensions was carefully constructed from the beginning. The careful choice of the sequence of flexible/rigid nodes and the family of modifications is described in the next part.

7.4.11 We define the class of strict allowed divisors $W_{k}$ on $\Gamma_{k}$ inductively as follows.

Assume first that $v_{k}$ is rigid, but at least one node of $\left(\Gamma_{k}, W_{k}\right)$ is flexible. Let $k^{\prime}>k$ be that flexible node for which $v_{k}, \ldots, v_{k^{\prime}-1}$ are all rigid. We modify $W_{k}$ such that we keep unmodified the restriction on $W_{k^{\prime}+1}$ and $i_{k^{\prime}, 1}$. We fix some $\ell \geq 2$ such that $d_{k^{\prime}, \ell} \nmid i_{k^{\prime}, \ell}$. Then we replace $i_{k^{\prime}, \ell}$ into $i_{k^{\prime}, \ell}+t d_{k^{\prime}, \ell}, t \in \mathbb{Z}$, but keep all other $i_{k^{\prime}, \ell}$ 's. Moreover, modify $i_{k^{\prime}} \mapsto i_{k^{\prime}}-t d_{k^{\prime}}$ too. Then $i_{k^{\prime}+1}$ and $i_{k^{\prime}, 1}$ will stay fixed.

This is the set of modifications we will refer to, and for strict divisors we impose the following properties. First, we assume that for all the possible modifications, the value $i_{k^{\prime}}$ is a multiple of $D_{k^{\prime}-1}^{*}$. Then, all these modifications can be extended by a rigid $v_{k^{\prime}-1}$ to $\Gamma_{k^{\prime}-1}$. Then we run again all the modifications (at $v_{k^{\prime}}$ ) and we assume that for all of them $D_{k^{\prime}-2}^{*} \mid i_{k^{\prime}-1}$. Then, again, all of them can be extended. We continue this, at the very end asking $D_{k}^{*} \mid i_{k+1}$ for all the modifications. If all these conditions are satisfied for $W_{k}$ then in all its modifications $\bar{W}_{k}$ the nodes $v_{k}, \ldots, v_{k^{\prime}-1}$ will be rigid, and we call $W_{k}$ strict. The strictness guarantees that when we run all the modifications at the level of $v_{k^{\prime}}$, all the divisors can be extended to some 
$W_{k}$. (Otherwise it might happen that for some modification and at some vertex both $d_{k^{\prime \prime}, 1} \nmid i_{k^{\prime \prime}, 1}$ and $D_{k^{\prime \prime}}^{*} \mid i_{k^{\prime \prime}+1}$ fail.)

From (7.4.10) we get

$$
i_{k}=\frac{i_{k^{\prime}}}{D_{k}^{*} \cdots D_{k^{\prime}-1}^{*}} \quad \text { and } \quad i_{k-1,1}=i_{k^{\prime}-1,1} D_{k}^{*} \cdots D_{k^{\prime}-1}^{*} .
$$

Since $i_{k^{\prime}-1,1} \mapsto i_{k^{\prime}-1,1}+t D_{k^{\prime}}$, the modifications induce

$$
i_{k} \mapsto i_{k}-\frac{t d_{k^{\prime}}}{D_{k}^{*} \cdots D_{k^{\prime}-1}^{*}} \quad \text { and } \quad i_{k-1,1} \mapsto i_{k-1,1}+t D_{k}^{*} \cdots D_{k^{\prime}-1}^{*} D_{k^{\prime}}
$$

If $v_{k}$ is flexible then $W_{k}$ is strict by definition. In fact the above discussion is valid in this case too with $k^{\prime}=k$. In particular, the set of modifications is given by $i_{k, \ell} \mapsto i_{k, \ell}+t d_{k, \ell}$ for the chosen $\ell$ and keeping the other $i_{k, \ell}$ 's, $i_{k} \mapsto i_{k}-t d_{k}$, $i_{k-1,1} \mapsto i_{k-1,1}+t D_{k}$.

If we run the above modification for the divisors $W_{m}$ constructed in Section 7.4.7, then $v_{m}$ stays stable, hence if the restriction of some $W_{k}$ to $\Gamma_{m}$ satisfies (1), then all its modifications keep satisfying (1).

In our procedure we consider only strict allowed divisors. They will be constructed inductively starting from the strict divisors $W_{m}$ constructed in Section 7.4.7. The inductive statement we prove is the following: for any $1 \leq k \leq m$ there exists a strict allowed divisor $W_{k}$ on $\Gamma_{k}$ satisfying $d_{k-1,1} \nmid i_{k-1,1}$, and (1) on $\Gamma_{m}$.

The proof of the inductive step breaks into two parts.

(a) If the above properties are true for some strict $W_{k+1}$ on $\Gamma_{k+1}$ then definitely it can be extended to an allowed divisor $W_{k}$, but this is not necessarily strict. We prove that by a good choice of one of its modifications, that divisor has a strict extension (not necessarily satisfying $\left.d_{k-1,1} \nmid i_{k-1,1}\right)$.

(b) If $W_{k}$ is strict and its restriction satisfies (1), then it can be replaced (by the above moves) by another strict divisor which satisfies both (1) and $d_{k-1,1} \nmid i_{k-1,1}$.

Note that part (b) provides the main inductive statement for $m=1$ too. Indeed, by Section 7.4 .7 a strict divisor $W_{m}$ with (1) exists, which by (b) can be replaced by a wanted one.

7.4.14 Here we prove part (a) of the inductive step Section 7.4.11.

Assume that $W_{k+1}$ is a strict divisor on $\Gamma_{k+1}$ satisfying (1) and $d_{k, 1} \nmid i_{k, 1}$. We consider all the modifications $\bar{W}_{k+1}$ of $W_{k+1}$ as in Section 7.4.11, and we distinguish the next two cases. 
First, suppose that there is no $\bar{W}_{k+1}$ (with or without $d_{k, 1} \nmid i_{k, 1}$ ) for which $D_{k}^{*} \nmid i_{k+1}$. Then we extend $W_{k+1}$ by a rigid node. The extended divisor $W_{k}$ will be strict.

Second, we assume that there exist some $\bar{W}_{k+1}$ with $D_{k}^{*} \nmid i_{k+1}$. The problem is that it might happen that in the new situation $d_{k, 1} \nmid i_{k, 1}$ fails, and the extension is not guaranteed. We claim that the two conditions $d_{k, 1} \nmid i_{k, 1}$ and $D_{k}^{*} \nmid i_{k+1}$ can be obtained simultaneously by some $\bar{W}_{k+1}$. Then we extend this new $\bar{W}_{k+1}$ to get a strict $W_{k}$ with flexible $v_{k}$.

Let us prove now the above claim.

Recall that $d_{k, 1} \nmid i_{k, 1}$. If $D_{k}^{*} \nmid i_{k+1}$ for $W_{k+1}$ then we are done. Similarly, if $d_{k, 1} \nmid i_{k, 1}$ for $\bar{W}_{k+1}$ then again we are done. Otherwise, by (7.4.13) we must have

$$
d_{k, 1} \nmid \delta:=D_{k+1}^{*} \cdots D_{k^{\prime}-1}^{*} D_{k^{\prime}} \quad \text { and } \quad D_{k}^{*} \nmid \Delta:=\frac{d_{k^{\prime}}}{D_{k+1}^{*} \cdots D_{k^{\prime}-1}^{*}} .
$$

We consider the modifications for $t=1,2,3$. Then either we get a wanted pair or we will have simultaneously

$$
\left\{\begin{array} { l } 
{ d _ { k , 1 } \nmid i _ { k , 1 } , } \\
{ D _ { k } ^ { * } | i _ { k + 1 } , }
\end{array} \quad \left\{\begin{array} { l } 
{ d _ { k , 1 } | i _ { k , 1 } - \delta , } \\
{ D _ { k } ^ { * } \nmid i _ { k + 1 } + \Delta , }
\end{array} \quad \left\{\begin{array} { l } 
{ d _ { k , 1 } \nmid i _ { k , 1 } - 2 \delta , } \\
{ D _ { k } ^ { * } | i _ { k + 1 } + 2 \Delta , }
\end{array} \quad \left\{\begin{array}{l}
d_{k, 1} \mid i_{k, 1}-3 \delta, \\
D_{k}^{*} \nmid i_{k+1}+3 \Delta .
\end{array}\right.\right.\right.\right.
$$

This implies $d_{k, 1} \mid 2 \delta$ and $D_{k}^{*} \mid 2 \Delta$. This together with (7.4.15) implies that both $d_{k, 1}$ and $D_{k}^{*}$ should be even. This is not possible since $d_{k, 1}$ and $D_{k}^{*}$ are relative prime.

7.4.16 Finally we prove part (b) of the inductive step Section 7.4.11.

Assume that $W_{k}$ is a strict allowed divisor on $\Gamma_{k}$ such that its restriction satisfies (1). If $v_{k}$ is rigid we will use all the notation of Section 7.4.11, where $v_{k^{\prime}}$ is the closest flexible node to $v_{k}$. In fact, these notation can also be used when $v_{k}$ is flexible, with the convention $k^{\prime}=k$.

We have to show that for some modification of $W_{k}$ one has $d_{k-1,1} \nmid i_{k-1,1}$. We assume that this is not the case, that is, for all modifications of $W_{k}$ at $v_{k^{\prime}}$ one has

$$
d_{k-1,1} \mid i_{k-1,1}=\left(i_{k^{\prime}-1,1}+t D_{k^{\prime}}\right) \cdot D_{k}^{*} \cdots D_{k^{\prime}-1}^{*},
$$

and we wish to get a contradiction.

For $\ell \in\left\{2, \ldots, n_{k}\right\}$ set $a_{\ell}:=\operatorname{gcd}\left(d_{k-1,1}, d_{k, \ell}\right), A^{*}:=\prod_{\ell \geq 2} a_{l}$ and $d_{k-1,1}^{\prime}:=$ $d_{k-1,1} / A^{*}$. About $d_{k-1,1}^{\prime}$ we wish to prove two facts. First, clearly

$$
d_{k-1,1}^{\prime} \mid\left(i_{k^{\prime}-1,1}+t D_{k^{\prime}}\right) \cdot D_{k+1}^{*} \cdots D_{k^{\prime}-1}^{*} .
$$


The second one is

(7.4.19) $d_{k-1,1}^{\prime} \in \mathcal{S}\left\langle\frac{D_{k+1}}{d_{k+1,2}}, \ldots, \frac{D_{k+1}}{d_{k+1, n_{k+1}}}, \frac{D_{k+1}^{*} D_{k+2}}{d_{k+2,2}}, \ldots, \frac{D_{k+1}^{*} D_{k+2}}{d_{k+2, n_{k+2}}}, \ldots\right)$.

The semigroup involved above is the semigroup associated with that diagram which is obtained from $\Gamma_{0}$ by deleting the star-shaped subdiagram around $v_{k}$. (In fact, (7.4.18) can also be interpreted in this way.) The proof of (7.4.19) runs as follows. Write

$$
d_{k-1,1}=\sum_{\ell \geq 2} m_{\ell} \frac{D_{k}}{d_{k, \ell}}+D_{k}^{*} \cdot \sum_{\ell \geq 2} n_{\ell} \frac{D_{k+1}}{d_{k+1, \ell}}+D_{k}^{*} D_{k+1}^{*} \cdot \sum_{\ell \geq 2} n_{\ell}^{\prime} \frac{D_{k+2}}{d_{k+2, \ell}}+\cdots .
$$

Then $a_{\ell} \mid m_{\ell}$, hence $A^{*} \mid m_{\ell} D_{k}^{*} / d_{k, \ell}$ too, for any $\ell \geq 2$. In particular, $d_{k-1,1}^{\prime}$ belongs to

$$
\begin{array}{r}
\mathcal{S}\left\langle d_{k, 1}, D_{k+1} / d_{k+2,1}, \ldots, D_{k+1} / d_{k+1, n_{k+1}}, D_{k+1}^{*} D_{k+2} / d_{k+2,1},\right. \\
\left.\ldots, D_{k+1}^{*} D_{k+2} / d_{k+2, n_{k+2}}, \ldots\right\rangle .
\end{array}
$$

But $d_{k, 1}$ is in the semigroup generated by the others (cf (7.4.3)), thus we get (7.4.19). The step how we get $d_{k-1,1}^{\prime}$ from $d_{k-1,1}$ can be continued. In the second step we set $d_{k-1,1}^{\prime \prime}:=d_{k-1,1}^{\prime} / \operatorname{gcd}\left(d_{k-1,1}^{\prime}, D_{k+1}^{*}\right)$. Dividing consecutively by the corresponding divisor of $D_{k}^{*}, \ldots, D_{k^{\prime}-1}^{*}$, from $d_{k-1,1}$ we get $\bar{d}_{k-1,1}$ with the following properties:

$$
\begin{aligned}
& \bar{d}_{k-1,1} \mid i_{k^{\prime}-1,1}+t D_{k^{\prime}}, \\
& \text { or, equivalently, } \bar{d}_{k-1,1} \text { divides both } i_{k^{\prime}-1,1} \text { and } D_{k^{\prime}},
\end{aligned}
$$

$$
\bar{d}_{k-1,1} \in \mathcal{S}\left\langle\frac{D_{k^{\prime}}}{d_{k^{\prime}, 1}}, \ldots, \frac{D_{k^{\prime}}}{d_{k^{\prime}, n_{k^{\prime}}}}\right\rangle \text {. }
$$

Now (7.4.21) together with $\bar{d}_{k-1,1} \mid D_{k^{\prime}}$, via Lemma 7.2.1(b), implies that for some $\ell_{0} \in\left\{1, \ldots, n_{k^{\prime}}\right\}$ one has $D_{k^{\prime}} / d_{k^{\prime}, \ell_{0}} \mid \bar{d}_{k^{\prime}-1,1}$. This with $\bar{d}_{k-1,1} \mid i_{k^{\prime}-1,1}$ implies that $D_{k^{\prime}} / d_{k^{\prime}, \ell_{0}} \mid i_{k^{\prime}-1,1}$. Then the formula (7.4.6) for $i_{k^{\prime}-1,1}$ implies that $d_{k^{\prime}, \ell} \mid i_{k^{\prime}, \ell}$ for $\ell \in\left\{1, \ldots, n_{k^{\prime}}\right\} \backslash\left\{\ell_{0}\right\}$, which contradicts the fact that $v_{k^{\prime}}$ is flexible.

7.4.22 Now we verify that the above construction provides infinitely many divisors $W_{k}$ at each step $k$. Indeed, when $k=m$ then in Section 7.4.7 there are infinitely many possibilities for $i_{m, 1}$ to realize a desired $W_{m}$. Furthermore, in the extension procedure, this initially chosen $W_{m}$ is modified, but the original $i_{m, 1}$ is never touched.

Moreover, we can obtain this way infinitely many (extended) effective divisors $W_{m}$. Indeed, in Section 7.4.7 we can choose the value $v_{m}$ freely modulo $N_{m}$, in particular positive and large enough with respect to all decorations along the edges of $\Gamma$. Then also $i_{m-1,1}$ and further $\left\{i_{m, \ell}\right\}_{\ell=1}^{n_{m}}$ can be chosen "large". In fact, if $v_{m}$ is large enough, 
then all constructed (and modified) multiplicities along dashed arrows, on $\Gamma_{1}$ and further on the whole of $\Gamma$, will be positive. This ends the proof of Proposition 7.4.1.

7.4.23 Theorem Let $(X, 0)$ be an IHS germ and $f$ an analytic function on $X$, such that the minimal embedded resolution diagram $\Gamma_{\pi}(X, f)$ satisfies the semigroup condition. Let $\lambda$ be a monodromy eigenvalue of $f$ at a point of $\{f=0\}$. Then there exist infinitely many (effective) allowed $P$-divisors $W$ for $(X, \operatorname{div}(f))$, and for each of them a pole $s_{0}$ of the topological zeta function $Z(f, W ; s)$ such that $\exp \left(2 \pi i s_{0}\right)=\lambda$.

Proof The proof of Theorem 6.3.1 is still valid here, replacing the use of Proposition 6.2.5 by Proposition 7.4.1.

7.4.24 Example We provide an example where the semigroup condition is not satisfied, and where a given eigenvalue cannot be induced by a pole of a zeta function associated to any allowed divisor.

We reconsider Example 3.1.15, but taking $F$ as the divisor corresponding to the unique arrowhead with multiplicity $N$ (instead of multiplicity 1 ).

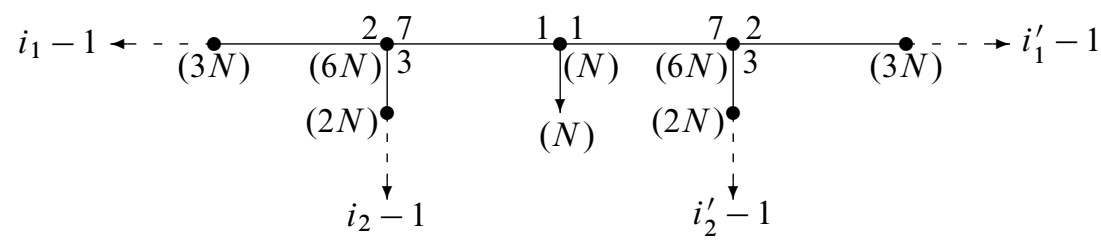

Now we have that the Alexander polynomial is $\Lambda(t)=\Delta_{1}(t)=\left(t^{2 N}-t^{N}+1\right)^{2}$. We take more specifically $N=7$, then $N_{1}=42$, and we pick $\lambda:=\exp (2 \pi i(-5 / 42)) \in$ Eig. Recall that (Example 5.1.11) $v_{1}=-78+7 I+6 I^{\prime}$, where $I=3 i_{1}+2 i_{2}$ and $I^{\prime}=$ $3 i_{1}^{\prime}+2 i_{2}^{\prime}$. We search for an allowed $W$ such that $-v_{1} / 42$ is a pole of $Z(F, W ; s)$ and $v_{1} \equiv 5(\bmod 42)$. This last condition is equivalent to $I \equiv 5(\bmod 6)$ and $I^{\prime} \equiv 1$ $(\bmod 7)$. But for $W$ to be allowed we need (cf Example 4.1.7) that $I=7$ or $I^{\prime}=7$. So such an allowed divisor $W$ does not exist. Note that also the node $v_{1}^{\prime}$ cannot induce $\lambda$ by the symmetric argument, and that the node $v_{0}$ cannot induce primitive $42-$ th roots of unity.

7.4.25 Example (a) We recall Example (3.5) of Rodrigues [24]. Consider the following resolution graph (the right graph below):
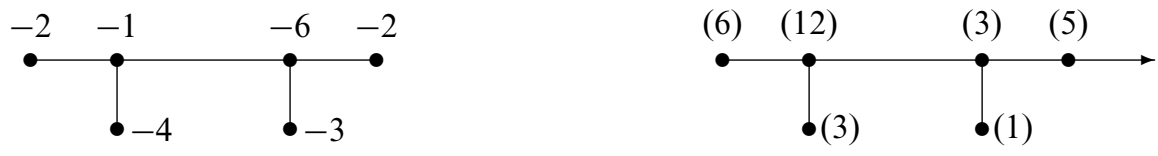
It is easy to verify that it is a numerically Gorenstein elliptic graph with length of the elliptic sequence two (for terminology, see eg [17]). It was not mentioned in [24], but this graph can be realized by a hypersurface isolated singularity with multiplicity 3 and geometric genus 2 (see also [33, case (24) in Table 4]). We consider the nowhere vanishing form $\omega_{0}$ on $X \backslash\{0\}$. The computation in [24] shows that, for the indicated Weil divisor $F$, the zeta function $Z\left(F, \omega_{0} ; s\right)$ has $s_{0}=1 / 3$ as a simple pole, but that $\exp (2 \pi i / 3)$ is not a root of the involved Alexander polynomial.

Note that this example does satisfy the (analogue of) the semigroup condition. Let us explain what we mean by this. Even if a graph is not unimodular, one can associate with it a splice diagram (by the very similar way as in Section 2.1), and one can impose the semigroup condition in the same way as above read from the splice diagram; cf [20]. For example, the "culpable" node with decoration -6 (which provides the counterexample to the "naively generalized" Monodromy Conjecture) satisfies the semigroup condition, since the determinant of the $(-2,-1,-4)$ string is 2 which is included in the semigroup generated by 2 and 4 . Nevertheless, for this graph the combination of Goals (1) and (2) fails. The point is that this graph is not unimodular, hence our main result does not apply to it.

This also shows that in our discussions the IHS-restriction is essential: any generalization of our main results to non-IHS germs requires the replacement of the semigroup condition by a much stronger assumption.

(b) One can ask if there is any unimodular graph providing a counterexample to the "naively generalized" Monodromy Conjecture. Here is one, again, in a combinatorial setting. The form is the standard Gorenstein form, whose analytic realization can easily be checked; the function-multiplicities are listed on the second diagram, where the analytic realization of the function is not guaranteed, and $N$ is a positive integer. The example shows that in our combinatorial arguments from this section the semigroup assumption cannot be eliminated.
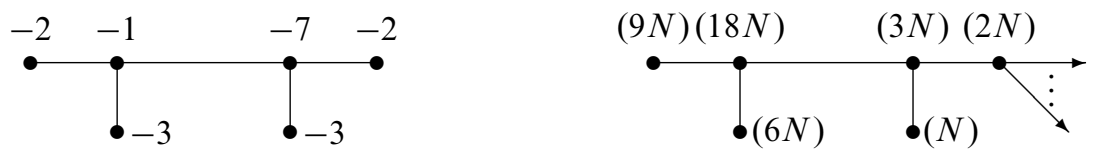

$N$ arrows

Clearly, the semigroup condition at the vertex with decoration -7 is not satisfied. (Indeed, the determinant of the $(-2,-1,-3)$ string is 1 , which is not an element of $\mathcal{S}\langle 2,3\rangle$.) By a computation one gets that $7 / 3 N$ is a pole of $Z(s)$, but $\exp (14 \pi i / 3 N)$ is not a root of

$$
\Delta_{1}(t)=\frac{\left(t^{9 N}+1\right)\left(t^{2 N}-1\right)^{N-1}(t-1)}{\left(t^{3 N}+1\right)\left(t^{N}-1\right)} .
$$




\subsection{Final remarks}

(a) (The definition of allowed forms revisited) There is a crucial feature regarding the definition of the allowed divisors: it does not use the multiplicity system of the divisor $F$, only its support. This has the following positive output: the family of allowed divisors can be defined uniformly for all divisors $F$ with the same support, and all the results we prove are valid uniformly for all these divisors $F$ (or, functions $f$ with the same support). To exemplify, let us rewrite Theorem 5.1.6 in the following way.

7.5.1 Theorem Let $(X, 0)$ be an IHS germ, and $F^{\prime}$ a reduced Weil divisor on $X$. Consider an allowed divisor $W$ associated with $\left(X, F^{\prime}\right)$. Then, for any function $g$ which has set-theoretical vanishing set $g^{-1}(0)=F^{\prime}$, and any pole $s_{0}$ of the topological zeta function $Z(g, W ; s), \exp \left(2 \pi i s_{0}\right)$ is a monodromy eigenvalue of $g$ at some point of $\{g=0\}$.

Note that the zeta-function $Z(F, W ; s)$ and the Alexander polynomial $\Lambda_{\Gamma(F)}(t)$ do depend essentially on the multiplicities of $F$.

The above new version Theorem 7.5.1 is definitely a much stronger statement than the original Theorem 5.1.6. The interested reader is invited to rewrite all the other results, especially Theorem 7.3.1 and Theorem 7.4.23 in the corresponding new versions. Of course, in order to do this, we have to observe that the definition of the semigroup condition associated with $\Gamma_{\pi}(X, F)$ too depends only on the support of $F$.

(b) (The restriction Section 2.3(2) of $W$ revisited) The restriction Section 2.3(2) (see also Section 1.5.2) was very convenient in the computations of arithmetical and numerical invariants, and additionally created a strong link between the supports of $F$ and $W$. Moreover, in that choice, we had in mind the analytic realization of the divisor $W$ too, that is, the applicability of the main results. More precisely, in general, it is a rather hard question to determine the analytic realization of some topologically identified arrowheads/divisors. For example [18] shows that simultaneous realization of some arrowheads is strongly obstructed. On the other hand, there is a "natural" family of analytic singularities for which the analytic realization of the class of arrowheads considered in Section 2.3(2) (arrowheads supported by boundary vertices) is automatically guaranteed. This is the class of "splice singularities"; cf [20;21]. In is worth to mention that the analytic realization of these germs is guaranteed by an arithmetical property of the graph $\Gamma$ (see the End Curve Theorem in $[22 ; 23]$ ), which is nothing else but the semigroup condition Section 2.1.4.

In this way, the simultaneous appearance of the restriction Section 2.3(2) regarding the divisors $W$, and of the semigroup condition might be natural. Moreover, for a 
considerably large class of examples, when the analytic realization of all the forms $W$ is guaranteed, the semigroup condition too will be satisfied (compare also with Section 7.1). This supports strongly the results of this section, and motivates once again the semigroup condition, showing that its appearance is not just a technical necessity (compare also with Example 7.4.25(b)).

\section{References}

[1] D Abramovich, K Karu, K Matsuki, J Włodarczyk, Torification and factorization of birational maps, J. Amer. Math. Soc. 15 (2002) 531-572 MR1896232

[2] N A'Campo, La fonction zêta d'une monodromie, Comment. Math. Helv. 50 (1975) 233-248 MR0371889

[3] E Artal Bartolo, P Cassou-Noguès, I Luengo, A Melle Hernández, Monodromy conjecture for some surface singularities, Ann. Sci. École Norm. Sup. 35 (2002) 605640 MR1981174

[4] E Artal Bartolo, P Cassou-Noguès, I Luengo, A Melle Hernández, Quasi-ordinary power series and their zeta functions, Mem. Amer. Math. Soc. 178, no. 841, Amer. Math. Soc. (2005) MR2172403

[5] D Barlet, Contribution effective de la monodromie aux développements asymptotiques, Ann. Sci. École Norm. Sup. 17 (1984) 293-315 MR760679

[6] N Budur, M Mustaţă, Z Teitler, The monodromy conjecture for hyperplane arrangements, Geom. Dedicata 153 (2011) 131-137 MR2819667

[7] J Denef, F Loeser, Caractéristiques d'Euler-Poincaré, fonctions zêta locales et modifications analytiques, J. Amer. Math. Soc. 5 (1992) 705-720 MR1151541

[8] J Denef, F Loeser, Motivic Igusa zeta functions, J. Algebraic Geom. 7 (1998) 505-537 MR1618144

[9] D Eisenbud, W Neumann, Three-dimensional link theory and invariants of plane curve singularities, Annals of Math. Studies 110, Princeton Univ. Press (1985) MR817982

[10] M Kashiwara, Vanishing cycle sheaves and holonomic systems of differential equations, from: "Algebraic geometry (Tokyo/Kyoto, 1982)", (M Raynaud, T Shioda, editors), Lecture Notes in Math. 1016, Springer, Berlin (1983) 134-142 MR726425

[11] H B Laufer, Normal two-dimensional singularities, Annals of Math. Studies 71, Princeton Univ. Press (1971) MR0320365

[12] A Lemahieu, L Van Proeyen, Monodromy conjecture for nondegenerate surface singularities, Trans. Amer. Math. Soc. 363 (2011) 4801-4829 MR2806692

[13] A Lemahieu, W Veys, Zeta functions and monodromy for surfaces that are general for a toric idealistic cluster, Int. Math. Res. Not. 2009 (2009) Art. ID rnn122, 11-62 MR2471295 
[14] F Loeser, Fonctions d'Igusa p-adiques et polynômes de Bernstein, Amer. J. Math. 110 (1988) 1-21 MR926736

[15] F Loeser, Fonctions d'Igusa p-adiques, polynômes de Bernstein, et polyèdres de Newton, J. Reine Angew. Math. 412 (1990) 75-96 MR1079002

[16] B Malgrange, Polynômes de Bernstein-Sato et cohomologie évanescente, from: "Analysis and topology on singular spaces, II, III (Luminy, 1981)", Astérisque 101, Soc. Math. France (1983) 243-267 MR737934

[17] A Némethi, “Weakly” elliptic Gorenstein singularities of surfaces, Invent. Math. 137 (1999) 145-167 MR1703331

[18] A Némethi, W D Neumann, A Pichon, Principal analytic link theory in homology sphere links, from: "Topology of algebraic varieties and singularities", (J I CogolludoAgustín, E Hironaka, editors), Contemp. Math. 538, Amer. Math. Soc. (2011) 377-387 MR2777831

[19] A Némethi, W Veys, Monodromy eigenvalues are induced by poles of zeta functions: the irreducible curve case, Bull. Lond. Math. Soc. 42 (2010) 312-322 MR2601558

[20] W D Neumann, J Wahl, Universal abelian covers of surface singularities, from: "Trends in singularities", (A Libgober, M Tibăr, editors), Trends Math., Birkhäuser, Basel (2002) 181-190 MR1900786

[21] W D Neumann, J Wahl, Complex surface singularities with integral homology sphere links, Geom. Topol. 9 (2005) 757-811 MR2140992

[22] W D Neumann, J Wahl, The end curve theorem for normal complex surface singularities, J. Eur. Math. Soc. 12 (2010) 471-503 MR2608949

[23] T Okuma, Another proof of the end curve theorem for normal surface singularities, J. Math. Soc. Japan 62 (2010) 1-11 MR2648226

[24] B Rodrigues, On the monodromy conjecture for curves on normal surfaces, Math. Proc. Cambridge Philos. Soc. 136 (2004) 313-324 MR2040577

[25] B Rodrigues, W Veys, Poles of zeta functions on normal surfaces, Proc. London Math. Soc. 87 (2003) 164-196 MR1978573

[26] W Veys, Poles of Igusa's local zeta function and monodromy, Bull. Soc. Math. France 121 (1993) 545-598 MR1254752

[27] W Veys, Zeta functions for curves and log canonical models, Proc. London Math. Soc. 74 (1997) 360-378 MR1425327

[28] W Veys, The topological zeta function associated to a function on a normal surface germ, Topology 38 (1999) 439-456 MR1660317

[29] W Veys, Zeta functions and “Kontsevich invariants" on singular varieties, Canad. J. Math. 53 (2001) 834-865 MR1848509 
[30] W Veys, Vanishing of principal value integrals on surfaces, J. Reine Angew. Math. 598 (2006) 139-158 MR2270570

[31] W Veys, Monodromy eigenvalues and zeta functions with differential forms, Adv. Math. 213 (2007) 341-357 MR2331246

[32] J Włodarczyk, Toroidal varieties and the weak factorization theorem, Invent. Math. 154 (2003) 223-331 MR2013783

[33] S S T Yau, Hypersurface weighted dual graphs of normal singularities of surfaces, Amer. J. Math. 101 (1979) 761-812 MR536041

A Rényi Institute of Mathematics, Hungerian Academy of Sciences Realtanoda u. 13-15, Budapest, 1053, Hungary

Departement Wiskunde, Katholieke Universiteit Leuven

Celestijnenlaan 200B, 3001 Leuven, Belgium

nemethi@renyi.hu, wim.veys@wis.kuleuven.be

Proposed: Richard Thomas

Received: 1 February 2011

Seconded: Walter Neumann, Jim Bryan Accepted: 26 August 2011 